University of Rhode Island

DigitalCommons@URI

Open Access Dissertations

2018

\title{
A GENOMIC APPROACH TO THE COMPLEX RELATIONSHIP BETWEEN AN APICOMPLEXAN ENDOSYMBIONT AND ITS HOST
}

Christopher Paight

University of Rhode Island, cpaight12@gmail.com

Follow this and additional works at: https://digitalcommons.uri.edu/oa_diss

\section{Recommended Citation}

Paight, Christopher, "A GENOMIC APPROACH TO THE COMPLEX RELATIONSHIP BETWEEN AN APICOMPLEXAN ENDOSYMBIONT AND ITS HOST" (2018). Open Access Dissertations. Paper 808. https://digitalcommons.uri.edu/oa_diss/808

This Dissertation is brought to you for free and open access by DigitalCommons@URI. It has been accepted for inclusion in Open Access Dissertations by an authorized administrator of DigitalCommons@URI. For more information, please contact digitalcommons-group@uri.edu. 
A GENOMIC APPROACH TO THE COMPLEX RELATIONSHIP BETWEEN AN APICOMPLEXAN ENDOSYMBIONT AND ITS HOST

BY

CHRISTOPHER PAIGHT

A DISSERTATION SUBMITTED IN PARTIAL FULFILLMENT OF THE REQUIREMENTS FOR THE DEGREE OF DOCTOR OF PHILOSOPHY

IN

BIOLOGICAL AND ENVIRONMENTAL SCIENCES

UNIVERSITY OF RHODE ISLAND

2018 


\section{DOCTOR OF PHILOSOPHY DISSERTATION}

$\mathrm{OF}$

CHRISTOPHER PAIGHT

APPROVED:

Dissertation Committee:

Major Professor Christopher Lane

Jason Kolbe

Ying Zhang

Nasser H. Zawia

DEAN OF THE GRADUATE SCHOOL

UNIVERSITY OF RHODE ISLAND

2018 


\begin{abstract}
Nephromyces, a genus in the phylum Apicomplexa, has recently been described as having a mutualistic relationship with its host: tunicates in the Molgulidae family (Saffo et al. 2010). If true, Nephromyces would be the only known example of a mutualistic apicomplexan genus. In addition to the possible switch to mutualism, Nephromyces is one of a few apicomplexan groups containing bacterial endosymbionts. To test the hypothesis that endosymbiotic bacteria facilitated the transition of Nephromyces from parasitism, the metabolic capabilities of Nephromyces and its bacterial endosymbionts need to be determined. The transition from obligate parasite to endosymbiont is predicted to involve different selective pressures leading to wide spread genomic changes. Identifying these changes will lead to a better understanding of the dynamics between the different biological players in this system.
\end{abstract}

Using data from Illumina HiSeq, we have assembled and annotated the transcriptomes of Nephromyces and Cardiosporidium cionae. Using data from a combination of platforms; Illumina MiSeq, HiSeq, and Pacific Biosciences, we have partially assembled a pan-genome for Nephromyces and have assembled the genomes of its bacterial endosymbionts. Using amplicon sequencing, we have estimated the genetic diversity and prevalence of multispecies infections of Nephromyces and its bacterial endosymbionts in its host Molgula manhattensis. In addition to the implementation of next-generation sequencing technologies, this work is also based on laboratory cultures and species isolation experiments.

With the aforementioned data we are able to describe the transcriptome 
of Nephromyces and Cardiosporidium as well as the genomes of all three bacterial endosymbionts, providing a basic overview of the metabolism of this system. Nephromyces and Cardiosporidium both encode a complete purine degradation pathway, which enables them to break uric acid into pyruvate and glycine, additionally Nephromyces is also able to create malate from uric acid. This could represent the primary route of carbon, nitrogen and energy acquisition in Nephromyces. The genomes of the bacterial endosymbionts are severely reduced, but relatively enriched for vitamin and amino acid biosynthesis (at least in the Betaproteobacteria and Bacteroidetes symbionts). It is likely that the bacterial endosymbionts are supplementing vitamins and amino acids to the limited diet of uric acid found in Nephromyces. Our amplicon data reveals that nearly all $M$. manhattensis are infected with multiple species of Nephromyces. The community of Nephromyces forms a tightly integrated system of metabolic interdependencies based of the different bacterial endosymbionts. 


\section{ACKNOWLEDGMENTS}

Without the support, guidance, and mentorship of my advisor Dr. Christopher Lane this work would not have been possible. The growth I have achieved both as a person and as a scientist is in large part due to his excellent mentorship. The input I received from my committee members, Dr. Jason Kolbe, Dr. Ying Zhang, and Dr. Marta Gomez-Chiarri was invaluable in the development of how I thought of this system.

I would also like to thank my colleagues in the lab for our scientific discussions and sharing new methods and strategies for dealing with problems. In particular I would like to thank Liz Hunter, Katie Neville, and Brandon Seah for

their direct contributions to this work, and Kristina Terpis for making the lab run smoothly.

I would never have gotten to this point without the dedication of Jennifer Paight in teaching me to read, the sense of scientific curiosity instilled by Daniel Paight, and the patience and encouragement from Rachelle Amundson. Their continued support has pushed me to reach my highest potential. I would also like to thank Dr. Therese Markow for giving me my start in science, and encouraging me to attend graduate school. Finally I would like to thank Robert August for getting me through the rough spots and bad days. 


\section{PREFACE}

The data chapters 2-4 have been prepared for submission as manuscripts and the manuscript format is in use. Chapter 2 has been submitted to the journal Genome Biology and Evolution (GBE) as a research article it includes the following sections in order; abstract, introduction, material and methods, results, discussion, acknowledgments, and references. Chapter 3 will be submitted to the journal Proceeding of the National Academy of Sciences (PNAS) as a research article it includes the following sections in order; abstract, introduction, results, discussion, material and methods, and references. Chapter 4 will be submitted to the Journal of Eukaryotic Microbiology (J.Euk.Microbiol.) as a research article it includes the following sections in order; abstract, introduction, material and methods, results, discussion, acknowledgments, and references. 


\section{TABLE OF CONTENTS}

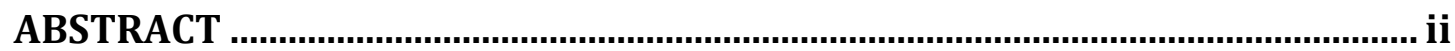

ACKNOWLEDGMENTS.................................................................................. iv

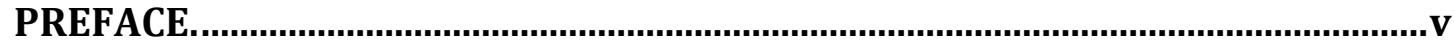

TABLE OF CONTENTS......................................................................................... vi

LIST OF TABLES .............................................................................................viii



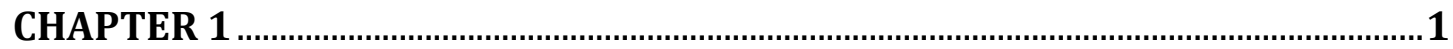

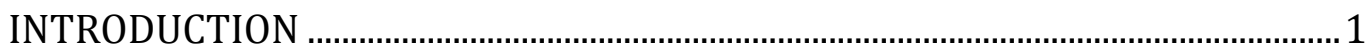



NEPHROMYCES ENCODES A URATE METABOLISM PATHWAY AND

PEROXISOMES, DEMONSTRATING THESE ARE NOT ANCIENT LOSSES

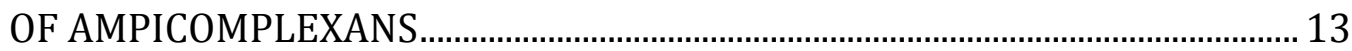

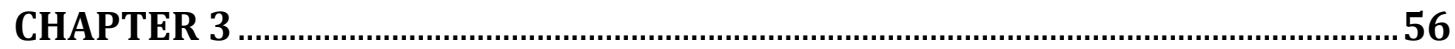

COMPARATIVE TRANSCRIPTOMICS OF TWO APICOMPLEXANS

(NEPHROMYCES AND CARDIOSPORIDIUM CIONAE) AND THE GENOMES OF

NEPHROMYCES'S BACTERIAL ENDOSYMBIONTS ........................................... 56

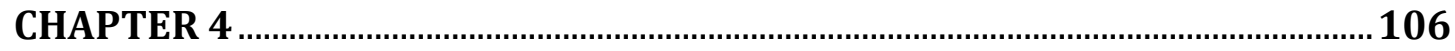

AMPLICON SEQUENCING REVEALS HYPER DIVERSITY AND UNIVERSAL MULTI-SPECIES INFECTION PREVALENCE IN THE GENUS

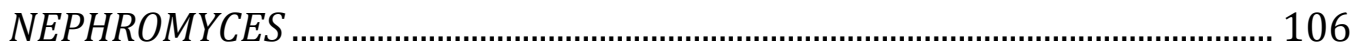

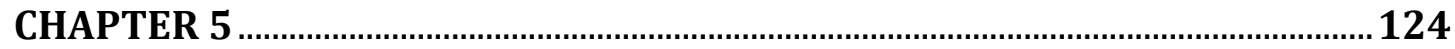




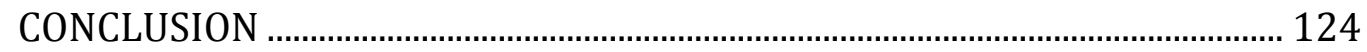

APPENDICES 


\section{LIST OF TABLES}

TABLE

Table 1. Nephromyces contigs annotated with genes involved with purine degradation.

Table 2. Genes involved in the biosynthesis and maintenance of peroxisomes as well as genes that are commonly targeted to peroxisomes.

Table 3. Percentile expression rank of purine degradation genes in Nephromyces, Cardiosporidium, Molgula manhattensis in both wild and laboratory grown populations.

Table 4. Genomic assembly statistics for Nephromyces bacterial endosymbionts. 75 


\section{LIST OF FIGURES}

FIGURE

PAGE

Figure 1. Maximum likelihood trees of genes involved in purine degradation.... 30

Figure 2. Proposed metabolism of uric acid in Nephromyces as a primary nitrogen, carbon and energy source.

42

Figure 3. Metabolic pathway capabilities of Nephromyces and its bacterial endosymbionts

Figure 4. Maximum likelihood trees of 16S rRNA for all three of Nephromyces

bacterial endosymbionts.

Figure 5. Collapsed Venn diagram of orthologous genes found in Nephromyces,

Cardiosporidium, Apicomplexa and chromerids 88

Figure 6. Venn diagram of orthologous genes found in Nephromyces,

Cardiosporidium, Toxoplasma, Cryptosporidium and Plasmodium 89

Figure 7. 18s rRNA amplicon species number per renal sac clustered at various

percent Identity levels. 113

Figure 8. COI amplicon species number per renal sac clustered at various percent Identity levels. 114

Figure 9. 16s rRNA amplicon species number per renal sac. 115

Figure 10. Fluorescence in situ labeled bacterial endosymbionts showing one type of bacterial endosymbiont per Nephromyces species. 


\section{CHAPTER 1}

\section{INTRODUCTION}

Apicomplexa is a large, diverse phyla thought to be composed entirely of parasites of metazoans (Morrison 2009). It is believed that every species of metazoan is host to at least one species of apicomplexan parasite. Some apicomplexans are of particular significance to humans including Plasmodium, the etiological agent of malaria. Plasmodium is estimated to have driven the evolution of 4.3 percent of the human genome (Whitfield 2002; McManus et al. 2017). Another apicomplexan of note is Toxoplasma, which is able to infect all warm-blooded animals and is estimated to infect a third of the global human population (Wilking et al. 2016). Cryptosporidium is a waterborne apicomplexan responsible for $90 \%$ of occurrences of severe diarrhea in children under five, and a continuing challenge for municipal water treatment (Sow et al. 2016). In addition to the medical significance of apicomplexans, there has been research into apicomplexans on the effects of parasitism on an organisms genome, due to their long 700-900 million year history as a lineage of obligate parasites (Kuo et al. 2008).

Obligate parasites face different challenges than free-living organisms, resulting in different evolutionary pressures and unusual life histories as well as dramatic genomic changes in parasitic lineages (Janouskovec \& Keeling 2016). One of the problems faced by parasites is the host's immune system. The need to evade the host's immune system results in a complex evolutionary arms race 
between host and parasite, and is a core component of the red queen hypothesis (van Valen 1973). Apicomplexans have developed numerous and diverse strategies for evading their host's immune system, including the var family of genes in Plasmodium. Var proteins are cell surface antigens which are capable of reorganizing into a wide number of protein conformations to keep the host from recognizing the infection (Kyes et al. 2007). Another strategy, used by Toxoplasma is to suppress immune response by silencing signal pathways and by forming the latent bradyzoite cyst stage, which causes chronic infection (Blader \& Saeij 2014).

In addition to evasion of the host's immune system, intracellular parasites have ready access to an abundance of pre-formed metabolites. Access to these pre-formed metabolites leads to one of the most common and pronounced consequences of parasite genome evolution, the loss of many basic biosynthesis pathways, which are critical in free-living organisms (Janouskovec \& Keeling 2016). This loss of biosynthesis capabilities is particularly pronounced in Apicomplexa. Amino acid biosynthesis, vitamin and cofactor biosynthesis, purine synthesis, purine degradation, and fatty acid biosynthesis have all been lost in Apicomplexa (Woo et al. 2015). Because these losses are observed throughout the phylum, it was believed that these losses occurred early in apicomplexan evolution. However, there has been a recent proposal that these losses are a continuous gradual process (Zarowiecki \& Berriman 2015). In either case, loss of biosynthetic pathways creates a dependence on the host for not only primary 
carbon and nitrogen, but also a dependence on salvaging the hosts premade metabolites. This has led to a number of intricate and elegant strategies for host manipulation.

As a consequence of the extraction of nutrients and metabolites, a parasite's growth and reproduction imposes a cost to their host. The total impact on the host by the parasite is known as virulence (Read 1994). If a parasite's virulence is too great the host will die, either directly due to the parasite, or as a consequence of being weakened; i.e., the host is too weak to find food, the host becomes easy prey for predators, or the weakened immune system makes the host vulnerable to other pathogens. If the host dies before the parasite can complete its life cycle, or before it can infect a new host, then the parasites fitness falls to zero. This leads to a complex balance between parasite growth and transmission to a new host. Factors involved in transmission and virulence include host genotype, parasite genotype, host health, parasite load, as well as external factors such as other parasites infecting the same host simultaneously (Frank 1996a). The interplay of these factors creates a dynamic relationship between host and parasite and has led to a wide variety of strategies (Alizon et al. 2009). One common strategy, adopted by many parasite species, is to lower their virulence to the host (Cressler et al. 2016). Parasites often achieve this by lowering their reproduction levels. Lower parasite density means lower virulence to the host, and as long as there is still good transmission to other hosts, this low-density strategy is often successful. A different strategy is 
exemplified by Plasmodium. Plasmodium merozoites proliferate within the host's liver cells through schizogony by simultaneously inhibiting cell death (thereby avoiding immunity) until parasite levels are high enough to cause cell death, releasing release sporozoites into the bloodstream. The high density of sporozoites overwhelms the immune system and creates a high likelihood that a mosquito feeding on the host will ingest blood with sporozoites, maximizing transmission success. After simultaneous release sporozoites re-infect the liver to repeat the cycle again. This cycle causes the episodic fevers seen in malaria patients. The episodic overwhelming of the host immune system causes the high virulence found with Plasmodium infections. Plasmodium falciparum is particularly virulent, even among Plasmodium species. Some of the virulence of $P$. falciparum has been attributed to the large number of other human parasites found in the same locations. With the presence of other parasites the likelihood of multiple parasitic infections increases, which then leads to the virulence of all pathogens present being cumulative. Rather than a long sustained infection with low probability of transmission over a longer time, with the possibility of host death from other pathogens, P. falciparum, has adopted a high density, high virulence infection combined with periods hidden from the immune system, which maximizes transmission over a short period of time.

Although transmission strategies are diverse there are predictable patterns of a disease's epidemiology and the type of strategy a parasite is using. Parasites with high virulence are characterized by low prevalence in a population 
or by low prevalence with sporadic outbreaks (Frank 1996b). Parasites using this strategy may reach high cellular densities in an effort to maximize transmission before the host dies. Alternatively, high-sustained prevalence in a host population indicates low virulence. Parasites using this strategy often maintain low cell densities with lower transmission rates over a longer period of time. Parasite virulence and transmission strategies are not dichotomous, but rather a continuum. Parasites with the highest, sustained prevalence being the least virulent and parasites with the lowest prevalence being the most virulent. Of course such predictions about virulence only apply to parasites that have coevolved with their host, and does not apply to parasites infecting a new or incidental host. In these instances, virulence is often extremely high and often results in the host death before transmission, leading to a self-limiting infection pattern, i.e. Ebola in humans.

The relationship between prevalence and virulence is important for this work because Nephromyces mutualistic relationship with their hosts, Molgula tunicates was solely based on the nearly $100 \%$ year round infection prevalence (Saffo et al. 2010). As infection prevalence only predicts virulence, and not host/endosymbiont relationship, this characterization may have been unfounded and premature. Characterization of mutualism based solely on infection prevalence for Nephromyces is particularly surprising, because it becomes the only mutualistic genus in a phylum composed entirely of parasites. However, there are other indicators, besides prevalence, suggesting that the relationship 
between Nephromyces and its host is unusual. Unlike other pathogens with low virulence, Nephromyces reaches extremely high cell densities inside its host. This high cell density becomes apparent when you compare Nephromyces to its closest known relative Cardiosporidium cionae, a blood parasite that infects a number of solitary tunicates outside of the Molgulidae family (Ciancio et al. 2008; Kumagai et al. 2010). As with Nephromyces, C. ciona has a high sustained infection prevalence reaching 95\% in Ciona intestinalis. Such high, sustained prevalence indicates that, like Nephromyces, C. ciona is largely avirulent. However, Nephromyces reaches over an order of magnitude higher cell densities than Ciona. In two such closely related organisms with closely related hosts with similar epidemiology in other respects, the difference in relative cell densities is striking. Typically, the higher the parasite load the greater the virulence, but paradoxically Nephromyces can remain avirulent and reach extremely high cell densities. Rather than focus on the proposed mutualistic relationship between Nephromyces and its host, which remains unclear, this work will focus on the apparent paradox in Nephromyces' epidemiology.

In order to consider the unusual epidemiology of Nephromyces, it is necessary to examine its other life history traits. The phylum Apicomplexa has a tremendous amount of variation in hosts, cell types infected, transmission methods, host manipulation strategies, life cycles, reproduction, and morphology (Roos 2005). Even with so much diversity, Nephromyces stands out as unusual for an apicomplexan. One of the most unusual aspects of Nephromyces' biology is 
where it lives. Nephromyces is only found and completes its entire life cycle inside the renal sac (Saffo 1982). Specifically, Nephromyces is found in the lumen of the renal sac and, unlike other apicomplexans, is extra-cellular, with no part of its life cycle inside or joined to its host's cells. The renal sac is a large, ductless, structure present only in tunicates in the Molgulidae family (Goodbody 1965). The function of the renal sac has not been determined, and despite its name, the renal sac does not function as a typical kidney (Saffo 1978). The renal sac was named for the large deposits of uric acid and calcium oxalate, nitrogenous compounds that are the major constituents of kidney stones (Saffo \& Lowenstam 1978). Localized deposits of uric acid are not exclusive to Molgula tunicates and many ascidians have crystallized uric acid deposits located in various tissues, but the deposits in Molgula are by far the largest (Lambert et al. 1998).

Another unusual aspect about Nephromyces is the presence of bacterial endosymbionts. Even though it is not unusual for Eukaryotes to have bacterial endosymbionts, it is unusual in the phylum Apicomplexa. The only other apicomplexan known to harbor a bacterial endosymbiont is $C$. ciona. Bacterial endosymbionts are a common way for an organism to add novel functionality to its metabolism, and the acquisition and maintenance of bacterial endosymbionts is a major driver of eukaryotic evolution Prominent examples include the alphaproteobacterium that became the mitochondria and the cyanobacterium that gave rise to the chloroplast (John \& Whatley 1975; Mereschkowsky 1905). More recent bacterial endosymbionts provide their hosts with a wide variety of 
metabolic capabilities including vitamin and co-factor biosynthesis, amino acid biosynthesis, methanogenesis, photosynthesis, and protection from parasitoids. (Moran et al. 2005; Gijzen et al. 1991; Marin et al. 2005; Hansen et al. 2012). These functions allow their hosts to colonize new habitats and take advantage of novel food sources.

One of the consequences of a parasitic lifestyle is the loss of biosynthetic capabilities, and bacterial endosymbionts can supplement a host's metabolism. It was hypothesized that Nephromyces bacterial endosymbionts were an important factor in Nephromyces' colonization of the renal sac and its paradoxical epidemiology (Saffo et al. 2010). Therefore, it was necessary to determine how Nephromyces bacterial endosymbionts were contributing to the host's metabolome. One hypothesis suggested that bacterial endosymbionts of Nephromyces are capable of degrading the abundant amounts of uric acid in the renal sac(Saffo et al. 2010). The degradation of uric acid was also proposed as the host benefit that made Nephromyces mutualistic instead of parasitic.

A previous study had found three different bacterial endosymbionts in Nephromyces: an alphaproteobacteria, a betaproteobacteria, and a bacteroidetes (Seah et al. 2011). This study also detailed how these different bacterial endosymbionts were never found together in the same Nephromyces cell. No explanation of how a species of a single-celled organism could maintain three different endosymbionts without the endosymbionts ever being together was given. What this study failed to recognize is there were multiple species of 
Nephromyces inside the same renal sac, and that different Nephromyces species contained a single type of bacterial endosymbiont (Chapter 4).

Organisms harboring multiple endosymbionts are not uncommon (Bennett \& Moran 2013; Moran et al. 2008; Gruwell et al. 2010). Many organisms that are dependent on bacterial endosymbionts contain two or three different endosymbionts. Multiple endosymbionts are often required due to the evolutionary consequences of a free-living bacteria becoming an endosymbiont (Wernegreen 2017, 2015; Mccutcheon \& Moran 2011; Moran 1996). One driver of bacterial endosymbionts' evolution is a tiny population size relative to freeliving bacterial. Another is when only a few, or just a single bacterium, is vertically transmitted to subsequent host generations. Small population size, coupled with extreme bottlenecks repeated every host generation, produces profound effects from genetic drift and results in an accelerated Muller's ratchet (Moran 1996). One of the consequences of the accelerated Muller's ratchet on bacterial endosymbionts is a severe reduction of all non-essential genes. Some of the genes commonly lost are DNA repair genes (Kuwahara et al. 2007). The loss of DNA repair genes combined with the effects of genetic drift leads to high mutation rates, a low ratio of synonymous/non-synonymous mutations, and an AT bias. Over time this results in endosymbiont genomes, which are small, gene poor, and AT rich (Moran 2002).

The genomic instabilities of bacterial endosymbionts can quickly make them a burden and a liability to the host. As bacterial endosymbionts decrease in 
function, the host must support their symbionts to a greater and greater degree. If a host is dependent on their symbionts, this can result in reduced fitness if the endosymbiont requirements outpace the host's ability to meet them. A common solution to the problem of symbiont degradation is acquiring additional bacterial endosymbionts. In such cases either the original bacterial endosymbiont is replaced in favor of the new endosymbiont, or both bacterial endosymbionts can be maintained together (McCutcheon \& Moran 2007). When both bacterial endosymbionts are retained, the metabolisms of the bacterial endosymbionts can become tightly intertwined. One such example from McCutcheon and Moran (2007) involves the endosymbionts of the glassy winged sharpshooter Homalodisca vitripennis, which maintains two bacterial endosymbionts Sulcia muelleria and Baumannia cicadellinicola. These two endosymbionts show tight metabolic integration, with S. muelleria capable of biosynthesizing 8 of 10 essential amino acids, menaquinone, and fabF for fatty acid biosynthesis. Baumannia cicadellinicola is capable of biosynthesizing the remaining two essential amino acids, a number of vitamins and cofactors, but not menaquinone, and has the rest of the genes needed for fatty acid biosynthesis except fabF (McCutcheon \& Moran 2007).

The genus Nephromyces contains three different bacterial endosymbionts, and although all three types of bacterial endosymbiont are regularly found in the same renal sac, no species of Nephromyces is known to contain more than one type of bacterial endosymbiont. It is currently unknown if the different 
endosymbionts are providing the same metabolic functions or not. If the bacterial endosymbionts are providing different functions, it is unclear if there is any interaction between the endosymbionts, as is seen in H. vitripennis. A significant difference between the two systems is that $H$. vitripennis contains multiple symbionts, and Nephromyces only contain one endosymbiont per organism. Given the very different habitats of Cardiosporidium and Nephromyces and the rarity of endosymbionts in apicomplexans, it seemed likely that the bacterial endosymbionts might have contributed to Nephromyces ability to colonize the renal sac and even to the unusual epidemiology of Nephromyces.

In order to explore; one, how Nephromyces is able to remain avirulent and reach such high cell densities, two, how Nephromyces was able to thrive in the unusual renal sac environment, three, what the relationship between Nephromyces and its host is, four, determine what effects this possibly mutualistic relationship had on Nephromyces genome, five, the role of the bacterial endosymbionts, and six, the differences between Nephromyces and other apicomplexans, we used a combination of next generation sequencing, culturing, and amplicon sequencing. Using Illumina HiSeq we sequenced and assembled the transcriptomes of Nephromyces, Cardiosporidium, their respective bacterial endosymbionts, Molgula manhattensis, and Ciona intestinalis (Chapter 2). Using a combination of Illumina MiSeq, HiSeq, and Pacific Biosciences we sequenced and partially assembled Nephromyces genome, and assembled two of Nephromyces bacterial endosymbionts genomes (alphaproteobacteria and bacteroidetes) 
(Chapter 3). Using amplicon sequencing targeting 18s rRNA, 16s rRNA, and COI, we identified the diversity of Nephromyces and its bacterial endosymbionts (Chapter 4). With these data, in combination with culturing and isolation experiments, we were able to make substantial progress on characterizing the biology of both Nephromyces and Cardiosporidium, as well as their bacterial endosymbionts. This includes the unusual epidemiology, how Nephromyces survives in the renal sac, the role of the bacterial endosymbionts, and how Nephromyces compares to Cardiosporidium and other apicomplexans. In addition, we have uncovered some unexpected results, including a highly unusual basis for an apicomplexan metabolism and genus specific co-dependent species complex. 


\section{Chapter 2}

Nephromyces encodes a urate metabolism pathway and peroxisomes, demonstrating these are not ancient losses of apicomplexans

by

Christopher Paight ${ }^{1}$, Claudio H. Slamovits ${ }^{2}$, Mary Beth Saffo ${ }^{3}$ \& Christopher E Lane $^{1 *}$

is submitted to the journal Genome Biology and Evolution

${ }^{1}$ Department of Biological Sciences, University of Rhode Island, Kingston RI, 02881, USA.

2 Department of Biochemistry and Molecular Biology, Dalhousie University, Halifax, Canada

${ }^{3}$ Smithsonian National Museum of Natural History, Washington, DC 20560, USA. 


\title{
CHAPTER 2
}

\author{
Abstract \\ The Phylum Apicomplexa is a quintessentially parasitic lineage, whose members \\ infect a broad range of animals. One exception to this may be the apicomplexan \\ genus Nephromyces, which has been described as having a mutualistic \\ relationship with its host. Here we analyze transcriptome data from Nephromyces \\ and its parasitic sister taxon, Cardiosporidium, revealing an ancestral purine \\ degradation pathway thought to have been lost early in apicomplexan evolution. \\ The predicted localization of many of the purine degradation enzymes to \\ peroxisomes, and the in silico identification of a full set of peroxisome proteins, \\ indicates that loss of both features in other apicomplexans occurred multiple \\ times. The degradation of purines is thought to play a key role in the unusual \\ relationship between Nephromyces and its host. Transcriptome data confirm \\ previous biochemical results of a functional pathway for the utilization of uric \\ acid as a primary nitrogen source for this unusual apicomplexan.
}

Key words: Apicomplexan, tunicates, Peroxisomes, Purine degradation, Nephromyces, Cardiosporidium

\section{Introduction}

Apicomplexans are most well known for being parasites of humans and livestock. Species in the genus Plasmodium, for instance, are the etiological agents of malaria. Apicomplexan species show tremendous variation in transmission 
methods, life cycles, host range, host manipulation strategies, cell-types infected, metabolic capabilities, immune evasion strategies, and virulence (Roos 2005; Reid et al. 2012; Kemp et al. 2013; Cardoso et al. 2016). Because of this variability, there are few apicomplexan characteristics shared throughout the phylum. Among the few universal apicomplexan features are a parasitic lifehistory and an inability to degrade purines (Janouskovec \& Keeling 2016). Nephromyces, a derived apicomplexan genus of uncertain phylogenetic placement, appears to be an exception to both of these traits.

Nephromyces was misclassified as a fungus for more than a 100 years, based on long hyphal-like cell structures, flagellated spores interpreted by some as chytrid zoospores and cell walls made of a chitin (Giard 1888). It was not until the application of molecular methods that Nephromyces was confirmed as a member of the derived apicomplexans (Saffo et al. 2010). Although some analyses have tentatively placed it sister to adeleids, coccidia, or piroplasmida, the precise phylogenetic position of Nephromyces remains unresolved (Saffo et al. 2010; Janouškovec et al. 2015). Nephromyces species are monoxenous (infecting a single host) and are found exclusively in the Molgulidae family of tunicates (Saffo \& Davis 1982). In a phylum composed of obligate parasites, the feature that distinguishes Nephromyces is its apparent mutualistic relationship with its tunicate hosts. The mutualistic relationship has been inferred based primarily on the nearly $100 \%$ infection rate and lack of clearance from the host ( Saffo 1978, 1988, 1990, Saffo et al. 2010). We use this label with caution, given how complex 
host-symbiont dynamics can be, how the costs and benefits of both "harmful" and "beneficial" symbioses can be difficult to determine, and how they can vary with genomic changes in hosts and symbionts (Leung \& Poulin 2008; Saffo 2104; Mushegian \& Ebert 2016).

A shift in lifestyle from obligate parasite to mutualistic symbiont is quite rare, and completely unknown from deep within a eukaryotic lineage with such a long evolutionary history of parasitism. One common consequence of a parasitic lifestyle is a loss of genes essential to free living organisms (Greganova et al. 2013; Janouškovec et al. 2015; Zarowiecki \& Berriman 2015; Petersen et al. 2015). In an intracellular environment, if precursor molecules can be scavenged, there is less selective pressure to maintain biosynthesis pathways, and many are consequently lost (Keeling 2004; Sakharkar et al. 2004; Morrison et al. 2007). In phyla such as Apicomplexa, these losses can be extreme and over half of the genes found in their photosynthetic sister group, chromerids, have been lost in apicomplexans (Woo et al. 2015).

With so many basic metabolic functions lost, and with such dependence on the host, it is difficult to see how the relationship between host and parasite could change to a mutualistic interaction. However, one way for an organism to rapidly change its metabolic capabilities is to take on a bacterial symbiont. Nephromyces has done just that, leading to the hypothesis that bacterial endosymbionts inside Nephromyces perform some of the metabolic functions lost in Apicomplexa, and potentially contribute something beneficial to the tunicate 
host (Saffo 1990; Saffo et al. 2010). Bacterial endosymbionts are common across the tree of life (although rare in apicomplexans) and perform a wide variety of functions for their hosts (Nowack \& Melkonian 2010). These include amino acid metabolism and vitamin metabolism (Moran et al. 2005), nitrogen metabolism (Lopez-Sanchez et al. 2009), defense (De Souza et al. 2009), chemotrophic energy production (Urakawa et al. 2005), and photosynthesis (Marin et al. 2005), to name a few.

A tempting hypothesis for the functional role of Nephromyces bacterial endosymbionts is the break down of purines to urea in the purine degradation pathway (Saffo 1990). In support of this hypothesis Nephromyces infected tunicates have quite high levels of the enzyme urate oxidase, which catalyzes conversion of uric acid to 5-hydroxyisourate, but the enzyme is undetectable in uninfected tunicates (Mahler et al. 1955; Saffo 1988). Coupled with the fact that all known apicomplexans and tunicates have lost the purine degradation pathway, these data were suggestive of a bacterial contribution to purine degradation.

In a yet unexplained quirk of tunicate biology, many tunicate species have localized deposits of uric acid (Lambert et al. 1998; Saffo \& Lowenstam 1978; Goodbody 1965). Storage as a form of excretion, nitrogen storage for future release, and structural support, are among the proposed functions of tunicate urate deposits (Goodbody 1965; Saffo 1988; Lambert et al. 1998). Tunicates in the Molgulidae family have the largest uric acid deposits, which are localized to a 
specialized, ductless structure, called a renal sac (Saffo \& Lowenstam 1978).

These uric acid deposits occur regardless of infection status, indicating a tunicate origin of these purine deposits. Despite the name, the renal sac has many features (most notably, the absence of any ducts or macroscopic openings) atypical for an excretory organ, and its biological function has yet to be determined.

Nephromyces infects feeding molgulid tunicates after the post-metamorphic onset of host feeding and completes its entire lifecycle within the renal sac. Four factors led to the conclusion that the bacterial endosymbionts within Nephromyces are the source of urate oxidase activity in this system: 1) the colonization of Nephromyces within a structure with high concentrations of urate, 2) the absence of urate oxidase activity in the molgulid hosts (Saffo, 1988, 1991), 3) the high urate oxidase activity found in Nephromyces (including its bacterial symbionts: Saffo, 1988, 1991), coupled with 4) the lack of obvious ultrastructural evidence of peroxisomes in Nephromyces (Saffo, 1990).

It is logical to think that the addition of bacterial endosymbionts to Nephromyces might have been key to colonizing this novel purine-rich niche, and is how Nephromyces escaped the "evolutionary dead end" of a parasitic lifestyle. In order to test this directly, and examine the metabolic relationships between the tunicate host, Nephromyces, and its bacterial endosymbionts, we sequenced the community transcriptome. To identify possible evolutionary or physiological changes involved in coevolution of Nephromyces with its molgulid hosts, we also sequenced the transcriptome of a sister taxon of Nephromyces, Cardiosporidium 
cionae (Ciancio et al., 2008; Saffo et al., 2010), an apicomplexan parasite found in the blood in a broad range of non-molgulid ascidian hosts, including Ciona intestinalis, Styela clava, Halocynthia roretzi, and Ascidiella aspersa (Ciancio et al. 2008; Dong et al. 2006). Interestingly, Cardiosporidium cionae also harbors bacterial endosymbionts, which allows for a more direct comparison between Nephromyces and Cardiosporidium.

Here we confirm the exceptionally high levels of urate oxidase activity in tunicates with Nephromyces, and extend this result to include high expression levels of all the genes in the purine degradation pathway (xanthine dehydrogenase, urate oxidase, 5-hydroxyisourate hydrolase, 2-oxo-4-hydroxy-4carboxy-5-ureidoimidazoline decarboxylase, and allantoinase). The breakdown of purines starts by conversion to xanthine. Xanthine then enters the ureide pathway and the enzyme xanthine dehydrogenase catalyzes the reaction of xanthine to urate (Xi et al. 2000; Nishino et al. 2008). Urate oxidase catalyzes the oxidation of uric acid to 5-hydroxyisourate. Following conversion the enzyme 5hydroxyisourate hydrolase catalyzes 5-hydroxyisourate to 5-hydroxy-2-oxo-4ureido-2,5-dihydro-1H-imidazole-5-carboxylate (Kahn \& Tipton 1998). This is further processed into (s)-allantoin by the enzyme 2-oxo-4-hydroxy-4-carboxy-5ureidoimidazoline decarboxylase (Jung et al. 2006). Allantoinase catalyzes (s)allantoin into allantoate. From this point there are a few different pathways with different endpoints that organisms are able to shuttle allantoate to (Cusa et al. 1999). A common end point is to process allantoate into urea and 
ureidoglycolate, to be further converted into carbon dioxide and ammonia. Alternatively, ureidoglycolate can be converted to glyoxylate, or the urea may be excreted as waste (Schultz et al. 2001; Werner et al. 2009).

We confirm that all the genes necessary for purine degradation are encoded by Nephromyces and Cardiosporidium, and not their endosymbiotic bacteria. Whereas the expression of urate oxidase by Nephromyces and Cardiosporidium is unexpected, a parallel issue is where the urate oxidase is physically located in the cell, given that apicomplexans reportedly lack peroxisomes (Schlüter et al. 2006). Urate oxidase activity is restricted to peroxisomes in eukaryotes, due to the numerous toxic byproducts that are produced in the break down of uric acid. Research into peroxisomes in Apicomplexa has a complex and contradictory history, with studies reporting both the presence (Kaasch \& Joiner 2000; Gabaldon et al. 2016) and absence (Ding et al. 2000; Schlüter et al. 2006; Gabaldon 2010) of peroxisomes in Apicomplexa. Recent work by Moog et. al (2017) and Ludewig-Klingner et al. (2018) demonstrates compelling support for peroxisomes in coccidians. Both studies present comprehensive bioinformatic (and also proteomic, in part) evidence for the presence of peroxisomal biogenesis factors (peroxins) and typical peroxisomal metabolic enzymes (including predicted relevant targeting signals) in coccidians (Moog et al. 2017; Ludewig-Klingner et al. 2018). However, neither paper provides explicit experimental evidence (for example, microscopic) for the formation of peroxisomes in these organisms. Although direct evidence is 
still absent, both studies point to (Lige et al. 2009)and their identification of peroxisome-like vesicles in T. gondii, for possible microscopic support.

Our data demonstrate that Nephromyces encodes a complete purine degradation pathway and a number of proteins predicted to be targeted to, or involved in, peroxisome biogenesis, maintenance and protein import, providing novel support of peroxisomes in Apicomplexa. Additionally, we propose the functional significance of purine degradation in Nephromyces, and reject the hypothesis that bacterial endosymbionts facilitated an escape from parasitism by providing genes in the purine degradation pathway.

\section{Methods}

Molgula manhattensis collection and laboratory culture

Molgula manhattensis tunicates were collected from a dock in Greenwich

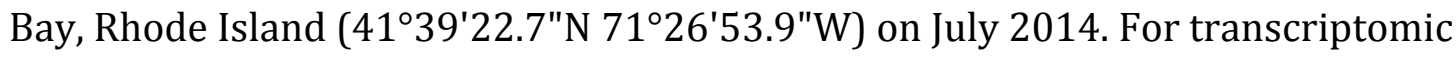
analysis, a single renal sac was separated from one tunicate, and all extraneous tissue removed. The intact renal sac was placed in liquid nitrogen for 5 min and then stored at $-80^{\circ} \mathrm{C}$ for later RNA extraction. Gonads were dissected from five, sexually mature, $M$. manhattensis, collected from the same population in Greenwich Bay, Rhode Island August 2014. Eggs and sperm were mixed with sterile seawater and divided evenly between two petri dishes. Plates were incubated at room temperature for two days with daily $100 \%$ water changes. Tunicate larvae attached to the bottom and sides of the petri dishes by day three. 
By day four, larvae had metamorphosed into adults and were actively feeding. Plates were moved to an incubator at $18^{\circ} \mathrm{C}$ with a $24 \mathrm{hr}$. dark cycle to limit growth of contaminants. Tunicates were fed by $100 \%$ water exchange with cultures of Isochrysis galbana and Chaetoceros gracilis three days a week. After several weeks tunicates were moved to aerated beakers to meet their increased nutrient and gas exchange requirements. Feeding regimen remained the same except that food volume was increased with tunicate growth. Tunicates were grown for six months until they were $\sim 10 \mathrm{~mm}$ across. Each renal sac was placed into a $1.5 \mathrm{ml}$ Eppendorf tube and flash frozen in liquid nitrogen. PCR screens confirmed Nephromyces was absent from lab-raised individuals. Lab grown tunicates were split into two groups. Renal sacs were harvested from three tunicates to use as transcriptome controls. A second group was infected with Nephromyces oocysts. Oocysts were collected from a wild M. manhattenensis and serially diluted by $50 \mathrm{x}$ to limit co-infections from multiple species, and raised for genomic analysis.

\section{Cardiosporidium cionae collection, isolation and concentration}

Ciona intestinalis were collected from docks in Snug Harbor RI $\left(41.3890^{\circ} \mathrm{N}\right.$, 71.5201 $\mathrm{W}$ ), in August 2017. Tunics were removed and the body wall was opened to allow access to the heart. A sterile syringe was used to remove cardiac blood as cleanly as possible. Blood was kept at $4^{\circ} \mathrm{C}$ until Cardiosporidium infection was verified using Giemsa stain to visualize Cardiosporidium. Heavily 
infected samples were pooled together and centrifuged at $500 \mathrm{~g}$ for 5 minutes. The resulting supernatant was removed and the samples were frozen in liquid nitrogen and stored at $-80^{\circ} \mathrm{C}$. Samples with low rates of infection were enriched for Cardiosporidium using sucrose gradients (Ogedengbe et al. 2015; Arrowood and Sterling 2016). Gradients of 20, 25, 30, 35, 40\% sucrose solutions in phosphate buffer were layered together. Approximately $5 \mathrm{ml}$ of tunicate blood was added to the column and centrifuged at $500 \mathrm{~g}$ for $30 \mathrm{mins}$ at $4^{\circ} \mathrm{C}$. The $25 \%$ and 30\% layers were collected (based on visual screens showing high Cardiosporidium cell density and low tunicate cell density), washed in PBS, pelleted and then frozen in liquid nitrogen and stored at $-80^{\circ} \mathrm{C}$.

\section{RNA Extraction}

RNA extraction buffer (Zymo Research LLC. Irvine, CA) was added to samples and ground with a pestle. Following grinding, the Zymo Quick-RNA kit (Zymo Research LLC. Irvine, CA) was used and the manufacturer's protocol was followed. RNA was converted to cDNA and sequenced at the School of Medicine Genome Resource Center, University of Maryland. Five separate paired-end RNA libraries (two from infected renal sac, and three uninfected renal sac) were multiplexed on one lane of the Illumina HiSeq platform, resulting in 326,299,923; $327,957,761$ and $316,754,780$ reads for the three renal sacs without Nephromyces, and 40,606,230 from the wild M. manhattensis renal sac. For Cardiosporidium, three samples of $C$. intestinalis blood were used: one with 
unseparated blood, one enriched with cells collected at the $25 \%$ sucrose gradient, and one enriched with cells from the $30 \%$ sucrose gradient were multiplexed on one lane of the Illumina HiSeq platform, resulting in 92,250,706; 109,023,104 and $110,243,954$ respectively. Transcriptome data was assembled and proteins were predicted with the Trinity/Trinotate pipeline version 2.4.0 run on the server at Brown University Center for Computation and Visualization (Haas et al. 2014). Reads assembled into 115,457; 388,535 109,446 contigs from infected, uninfected samples, and C. intestinalis respectively. Protein sequences were predicted using Transdecoder (Haas et al. 2014). Transcriptome completeness was assessed with Busco v3 against the Eukaryotic reference data sets (Simão et al. 2015).

\section{Genomic DNA Extraction}

The renal sacs from 8 lab grown M. manhattensis individuals were dissected and their renal fluid was pooled in a 1.5ml Eppendorf tube. Contents were centrifuged at $8000 \mathrm{~g}$ for $5 \mathrm{~min}$ to pellet Nephromyces cells, and following centrifugation the renal fluid was discarded. Five hundred microliters of CTAB buffer with $5 \mathrm{ul}$ of proteinase $\mathrm{K}$ and ceramic beads were added to the pelleted Nephromyces cells. The sample was placed in a bead beater for $3 \mathrm{~min}$. and then on a rotator for $1.5 \mathrm{hrs}$ at room temp. Five hundred microliters of chloroform was added, mixed gently and centrifuged for $5 \mathrm{~min}$. The top layer was removed and $2 \mathrm{x}$ the sample volume of ice cold 100\% EtOH and 10\% sample volume of 3M sodium acetate were added to the sample and incubated a $-20^{\circ} \mathrm{C}$ overnight. The sample 
was centrifuged at $16000 \mathrm{Xg}$ for $30 \mathrm{~min}$. and the liquid was removed. Ice cold $70 \%$ EtOH was added and centrifuged at 16000xg for $15 \mathrm{~min}$. Liquid was removed and sample air dried for $2 \mathrm{~min}$. DNA was re-eluted in $50 \mathrm{ul}$ of deionized water.

Illumina Sequencing

A nanodrop (2000c, ThermoScientific) was used to assess DNA purity and DNA concentration, and an agarose gel was run to assess genomic DNA fragmentation. Following quality control, an Illumina library was constructed. Library prep and sequencing were done at the URI Genomics and Sequencing Center (URIGSC). The completed library was sequenced on the Illumina MiSeq platform at the URIGSC and the HiSeq platform at the University of Baltimore sequencing center on three lanes.

\section{Pacific Biosciences Sequencing}

Using the contents of 150 (done in batches of 10 then pooled) $M$. manhattensis renal sacs, the same DNA extraction protocol was performed as for Illumina sequencing. DNA was sequenced using three SMRT cells on the Pacific Biosciences platform at the University of Baltimore sequencing center.

Illumina sequence data assembly

One MiSeq lane and three lanes of HiSeq, all from the same library, were trimmed using Trimmomatic (Bolger et al. 2014) and then assembled using Spades assembler (Bankevich et al. 2012) on the URI server BlueWaves.

Pacific Biosciences sequence data assembly

Pacific Biosciences reads were error corrected using pbsuite/15.8.24 
(English et al. 2012) on the Brown University server, Oscar. Reads were then assembled using Canu (Koren et al. 2014). Contigs generated by Canu were combined with Illumina MiSeq/HiSeq short reads with Abyss v2.02 (Jackman et al. 2017).

\section{Sequence annotation}

Genes in the urate pathway were identified initially using KEGG GhostKOALA and KASS and subsequently by BLASTP searches against NCBI's nr protein database (Kanehisa et al. 2016). All candidate genes were screened using InterProScan to predict function (Finn et al. 2017). A curated database of phylogenetically representative species with good quality annotations for the three purine degradation genes and malate synthase were downloaded from NCBI. These genes were then used to construct gene trees.

Sequences were aligned with MAFFT (Katoh \& Standley 2013) using FFTNS-i. Maximum likelihood phylogenetic trees were constructed performed with RAxML (Stamatakis 2014) using the GAMMA model with 1000 seed trees and 1000 bootstrap replicates. Trees were viewed and modified using Figtree (v1.4.0, http://tree.bio.ed.ac.uk/software/figtree/).

Protein sequences were used to search against PeroxisomeDB (Schlüter et al. 2009) and BLAST hits lower than $\mathrm{e}^{-20}$ were retained and used in a BLASTP query against NCBI's Refseq protein database (Schlüter et al. 2009). Additional peroxisomal genes were identified with KAAS (Moriya et al. 2007). As many of 
these peroxisome genes are encoded by M. manhattensis, all copies that had a closest hit to opisthokonta or bacteria were removed. Transcripts from uninfected M. manhattensis were used to screen additional tunicate genes using cd-hit at a 90\% identity level (Li \& Godzik 2006). Remaining genes were tested for signal motifs and subcellular location predictions with Wolf PSORTII, Ppero, TargetP, topcons, and Predotar (Supplementary Table 1)(Nakaia \& Horton 1999; Wang et al. 2017; Emanuelsson et al. 2007; Bernsel et al. 2009; Small et al. 2004).

Nephromyces specific RNAseq reads were mapped to our genomic assembly using bowtie2 (Langmead \& Salzberg 2012) with the -very sensitive flag set. Following mapping, Bedtools (Quinlan \& Hall 2010) was used to quantify coverage across contigs, which were separated based on coverage levels. Contigs identified as Nephromyces were annotated using Maker2 with ab initio gene predictions from Augustus (Holt \& Yandell 2011; Stanke et al. 2004).

\section{Results}

The contents of a single renal sac from an individual Molgula manhattensis resulted in 195,694 transcripts from M. manhattensis, Nephromyces, and the bacterial endosymbionts. After binning by species, 60,223 transcripts were attributed to Nephromyces. The cardiac fluid from 40 infected Ciona intestinalis individuals resulted in 109,446 transcripts, including 15,541 Cardiosporidium transcripts. The BUSCO algorithm was used to assess the completeness of the transcriptomes and reported $81.8 \%$ complete transcripts and $6.3 \%$ partial for the Nephromyces data and $69.7 \%$ complete and $11.9 \%$ partial for Cardiosporidium. 
The Nephromyces genome assembly consisted of 1176 contigs greater than $5 \mathrm{~kb}$ with a maximum length of $287,191 \mathrm{bp}$ and an average length of $36 \mathrm{~kb}$ (Paight et al, in prep). This dataset was used to search for purine degradation genes to determine their genomic context. All of the purine degradation genes, as well as malate synthase, were predicted and annotated in the genome by Maker2. All genes but URAD contained introns, and neighboring genes on the identified contigs had top BLAST hits to apicomplexans in all cases (Table 1), indicating that they are encoded in the Nephromyces genome, not the endosymbiotic bacteria or host Molgula manhattensis. Phylogenetic trees for xanthine dehydrogenase, uric oxidase, malate synthase and allantoicase consistently resolved the monophyly of Nephromyces, Cardiosporidium, and Chromerids (Figure 1). Chromerids are the photosynthetic and the closest free-living relatives of Apicomplexa (Moore et al. 2008), indicating a vertical inheritance of this pathway from the common ancestor of apicomplexans.

The presence of urate oxidase also provides further support for peroxisomes in some lineages of Apicomplexa (Moog et al. 2017; LudewigKlingner et al. 2018), because urate oxidase activity is confined to peroxisomes in eukaryotes (Usuda et al. 1994). In addition to urate oxidase, Nephromyces and Cardiosporidium encode more peroxisome-associated proteins than Plasmodium, and nearly the same complement of genes encoded by Toxoplasma (Table 2). There are a few notable differences between Toxoplasma and Nephromyces/Cardiosporidium, including the absence of PEX3, PEX16, VLACS, 
Table 1. Genomic context of the annotated purine degradation genes and malate synthase, in the Nephromyces genomic assembly. The phylogenetic affiliation of neighboring genes on each contig was identified by top hit against the NCBI nr database using BLASTp. Every contig encoding a target gene included other apicomplexan genes, and genes that did not hit apicomplexans had no strong affinity for other organisms.

\begin{tabular}{|c|c|c|c|c|c|}
\hline Gene & $\begin{array}{c}\text { Introns in } \\
\text { gene }\end{array}$ & Contig & $\begin{array}{c}\text { Contig length } \\
(\mathrm{kb})\end{array}$ & $\begin{array}{c}\text { Predicted } \\
\text { Genes on } \\
\text { Contig }\end{array}$ & $\begin{array}{c}\text { Genes with top } \\
\text { apicomplexan } \\
\text { BLAST hits }\end{array}$ \\
\hline XDH & 4 & Neph_3686418 & 24.5 & 4 & 2 \\
\hline UO & 7 & Neph_3687015 & 82.5 & 12 & 7 \\
\hline uraH & 2 & Neph_3685393 & 94.3 & 6 & 4 \\
\hline URAD & 0 & Neph_3687674 & 30.9 & 6 & 5 \\
\hline ALLC & 10 & Neph_3687655 & 116.3 & 16 & 11 \\
\hline MS & 6 & Neph_3671841 & 7 & 2 & 1 \\
\hline
\end{tabular}



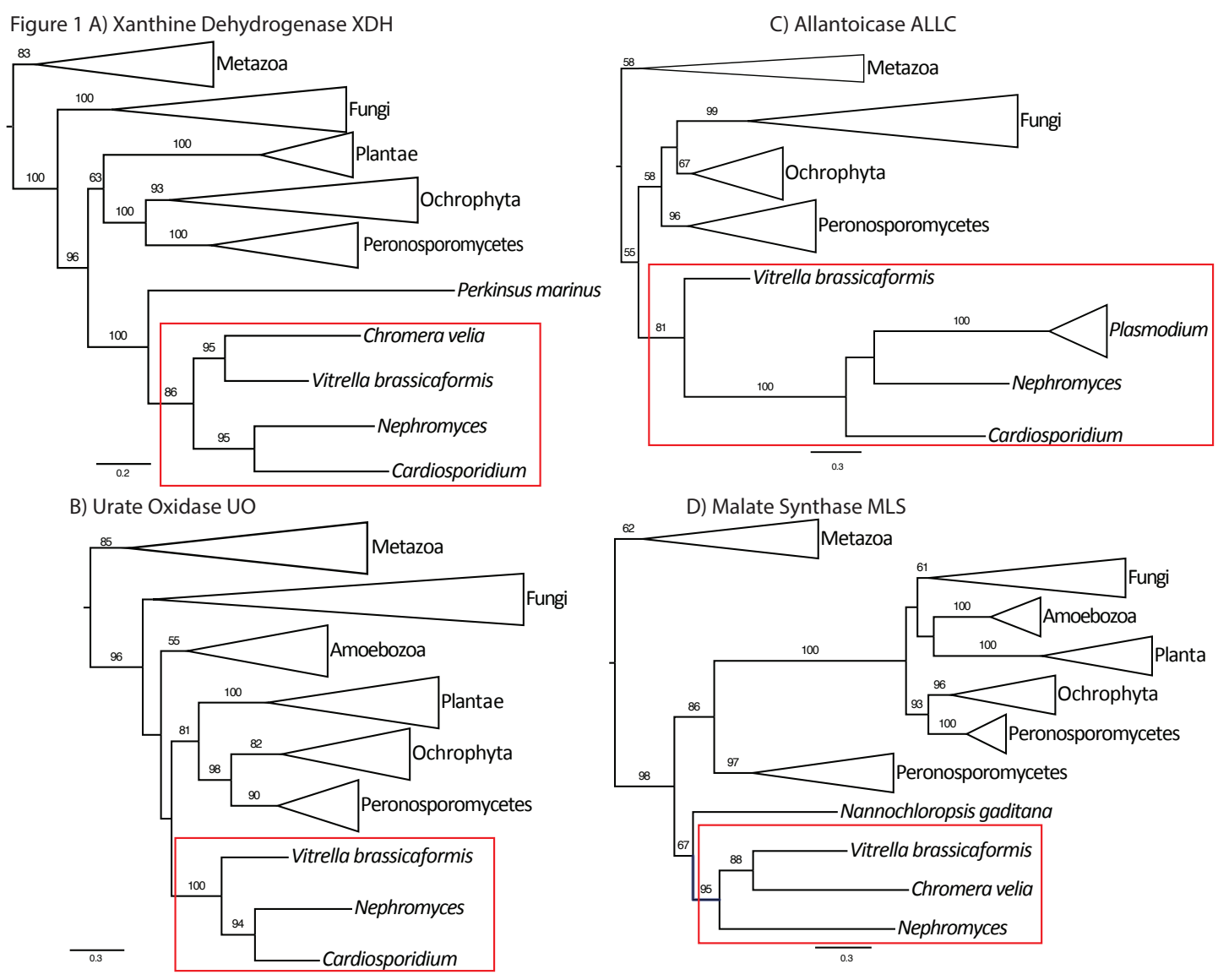

Figure 1. Maximum likelihood protein trees of A) Xanthine

Dehydrogenase, B) Urate oxidase, C) Allantoicase D) Malate synthase.

Genes A-C are involved in purine degradation and their position supports an ancestral apicomplexan purine degradation pathway in Nephromyces/Cardiosporidium. Malate synthase, D), acts on glyoxylate and acetyl-CoA to produce malate to complete the pathway. Stramenopiles are paraphyletic in the malate synthase phylogeny, possibly indicating a deep gene duplication. Whereas the support for deeper nodes is variable among all four genes, there is consistent support for a monophyletic origin of Nephromyces/Cardiosporidium genes with Chromerids (red box). Major lineages have been collapsed for presentation. Support values are percentage bootstrap support above $50 \%$. 
Table 2. Peroxiomal genes identified in Nephromyces and Cardiosporidium and their functional catagory. (X) denotes presence of gene and (-) absence. Vitrella brassicaformis (Vb) Chromera velia (Cv) Cardiosporidium (C) Nephromyces (N) Toxoplasma gondii (Tg) Plasmodium falciparum (Pf) Cryptosporidium parvum (Cp). Table modified based on Ludwig-Klinger et al. (2017).

Glyoxylate pathway

Peroxisome

Fatty acid oxidation a-oxidation

B-oxidation

\section{Protein}

Isocitrate lyase
Malate synthase
Citrate synthase
Aconitase
Malate dehydrogenase

Biogenesis factor 1 Biogenesis factor 2 Biogenesis factor 3 Ubiquitin carrier protein Biogenesis protein 5 Biogenesis protein 6 Biogenesis protein 7 Biogenesis protein 10 Biogenesis factor 11 Biogenesis protein 12 Biogenesis factor 13 Membrane protein 14 Membrane protein 15

Biogenesis factor 16

Membrane protein receptor Biogenesis protein 22

Biogenesis factor 26

Membrane channel

Membrane protein 4

ATP/ADP-transporter

Fatty acid ABC-transporter

ROS metabolism

Protein

2-Hydroxyacyl-CoA lyase Phytanoyl-CoA hydrolase a-Methylacyl-CoA-racemase Acyl-CoA-oxidase

Multifunctional protein

Sterole carrier protein 2
Abbr.

Vb Cv C N Tg Pf Cp

$\mathrm{ICL}$

MLS

CS

ACO

$\mathrm{MDH}$

Pex1

Pex2

Pex3

Pex4

Pex5

Pex6

Pex7

Pex10

Pex11

Pex12

Pex13

Pex14

Pex15

Pex16

Pex19

Pex22

Pex26

PMP22

PMP27

PMP34

PMP70

MPV17

Abbr.

HPCL2

PHYH

AMACR

ACOX

DBP

SCPX



$x \quad x \quad x \quad x-$

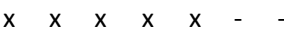

$x \quad x--x-$



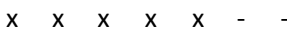

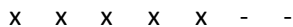

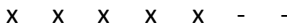



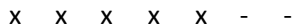

$\mathrm{x} x-\mathrm{x} x-$

$-\quad-\quad-\quad-\quad-$

$\begin{array}{lllllll} & \mathrm{X} & \mathrm{X} & \mathrm{X} & -\end{array}$

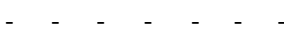

$x \quad x--x-$

$x-1-2--$

$\mathrm{x} \quad \mathrm{X} \quad \mathrm{X} \quad \mathrm{x} \quad \mathrm{x} \quad \mathrm{x} \quad \mathrm{x}$

$-\quad-\quad-\quad-\quad-\quad$

$\mathrm{x} \times \mathrm{x} \times \mathrm{-}-$

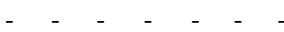

$\begin{array}{lllllll} & x & X & x & x & - & -\end{array}$

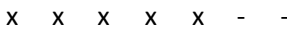



Vb Cv $C \quad N$ Tg Pf $C p$

$\begin{array}{ccccccc}- & x & - & - & - & x & - \\ x & x & - & - & - & - & - \\ - & - & - & - & - & - & - \\ x & x & x & x & x & - & - \\ x & x & x & x & x & - & - \\ - & x & - & - & x & - & -\end{array}$


Other oxidation

Amino acid metabolism

Antioxidant system

Multifunctional protein

Acetyl-CoA acyltransferase 1

PBE

2, 4-dienoyl-CoA reductase

$d(3,5)$ - $d(2,4)$-dienoyl-CoA isomerase $E C H$

ATP-binding cassette, subfamily $D$

Long-chain acyl-CoA synthetase

Solute carrier family 27, member 2

Acyl-CoA thioesterase 8

Nucleoside disphosphate-linked $\mathrm{m}$.

Multifunctional protein

D-Amino-acid oxidase

Isocitrate dehydrogenase

N1-acetylpolyamine oxidase

L-Pipecolate oxidase

hydroxymethylgluatryl-CoA lyase

(S)-2-hydroxy-acid oxidase

Hydrogen peroxide metabolism Catalase

Superoxide dismutase

Nitric-oxide synthase, inducible

Peroxiredoxin 1

Peroxiredoxin 5

Glutathione metabolism

Glutathione S-transferase kappa 1 Protein

Etherphospholipid biosynthesis

Dihydroxyacetone phosphate acyltr. DHAPAT

Alkyldihydroxyacetonephosphate syn AGPS

Fatty acyl-CoA reductase

Purine metabolism

Xanthine dehydrogenase

Urate oxidase

Retinol metabolism

Sterol precursor biosynthesis

Dehydrogenase/reductase SDR family DHRS4

Mevalonate kinase

Phosphomevalonate kinase

FAR

$\mathrm{XDH}$

UO



$\begin{array}{llllllll} & x & x & x & x & x & - & -\end{array}$

$\begin{array}{llllll}x & x & x & x & x & x\end{array}$

VLACS $x \quad x-\frac{x}{\text { PTE }}-$

PTE $\quad-\quad-\quad-\quad-\quad-$

NUDT19 - $\mathrm{x}-\mathrm{-}-\mathrm{-}-$

AGT $\quad x \quad x \quad x \quad x \quad x \quad-$

DAO $\quad-\quad-\quad-\quad-\quad-\quad-\quad-$

IDH $\quad x \quad x \quad x \quad x \quad x \quad x \quad-$

PAOX $x \quad x-5-5$



HMGCL $\quad x \quad x \quad x \quad x \quad x \quad x \quad r$.

HAO $\quad x \quad x \quad x \quad x-$

CAT $x-x \quad x \quad x-\frac{1}{2}-$

SOD $\quad-\quad x \quad x \quad x \quad x$

INOS - $-\quad-\quad-\quad-\quad-$

PRDX1 $\quad x \quad x \quad-\quad x \quad x \quad x \quad x$

PRDX5 - $\quad-\quad-\quad-\quad-\quad-$

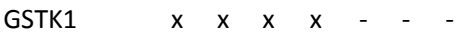

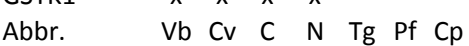

$\begin{array}{llllll} & x & x & x & x & -\end{array}$

$x \times x--\quad-$

$x \quad x-x \quad x--$

$\begin{array}{lllllll} & x & x & x & - & -\end{array}$

$x \quad x \quad x \quad x \quad-$

X $\mathrm{x} \times \mathrm{x}-\mathrm{-}$

MVK $\quad-\quad-\quad-\quad-\quad-\quad-\quad-$

PMVK 
and SCPX in Nephromyces/Cardiosporidium and the absence of PMP22, GSTK1, DHRS4, XDH, and UO in Toxoplasma. Additionally, Nephromyces encodes a copy of Malate synthase (MLS) absent in both Cardiosporidium and Toxoplasma. Malate synthase is a key gene in the glyoxylate cycle, a pathway maintained in the photosynthetic Chromera velia and Vitrella brassicaformis, but lost in all other apicomplexans (Ludewig-Klingner et al. 2018). Nephromyces/Cardiosporidium also encode the enzyme serine-pyruvate transaminase (AGXT), which also uses glyoxylate as a substrate. AGXT converts glyoxylate into glycine and pyruvate and is often localized to peroxisomes, however the localization of AGXT in Nephromyces/Cardiosporidium is unclear (Supplementary Table 1).

\section{Discussion}

The recent scrutiny by Moog et al (2017), and Ludewig-Klingner et al. (2018) has built a case for the presence of peroxisomes in some apicomplexan lineages. While some apicomplexans may have lost peroxisomes, it seems likely that this loss is not a universally shared trait in the phylum. Despite the extensive search for peroxisome-associated functions in apicomplexans, no genes involved in purine degradation were found in other sequenced apicomplexan genomes, with the lone exception of allantoicase in Plasmodium (Gardner et al. 2002). Our in silico predictions indicate a complete purine degradation pathway in Nephromyces and Cardiosporidium. In addition to highly expressed transcripts for 
the genes involved, all of the identified purine degradation genes and MLS have been located on genomic contigs from Nephromyces. Based on neighboring genes and the presence of introns in the Nephromyces genes matching the expressed transcripts, these contigs almost certainly originate from the Nephromyces genome (Table 1). Additionally, none of the purine degradation transcripts attributed to Nephromyces were detected in uninfected tunicates (Table 3). Phylogenetic trees of purine degradation genes are poorly supported at an interphylum level, indicating a rapid evolutionary rate. Whereas most genes are phylogenetically uninformative across the spectrum of eukaryotes, these gene trees have strong support for monophyly of purine degradation genes from Nephromyces and Cardiosporidium with Chromerids (Figure 1). The combination of gene trees, expression only when Nephromyces is present, and preliminary genomic assemblies strongly suggest that these genes were present since the divergence of Apicomplexa and Chromerida and have been vertically transmitted. Thus, these genes have been subsequently lost across apicomplexans, possibly multiple times. Although the exact placement of Nephromyces and Cardiosporidium is not certain (Saffo et al. 2010), multi-gene phylogenies place them in the subclass Hematozoa (Muñoz et al. in prep), suggesting that purine degradation was independently lost multiple times in Apicomplexa as well as maintained long after apicomplexans had become obligate parasites.

The presence of predicted purine degradation genes in Nephromyces and Cardiosporidium, adds a function not previously demonstrated in apicomplexan 
peroxisomes (Table 2; Moog et al 2017; Ludewig-Klingner et al. 2018). While Toxoplasma and Cardiosporidium/Nephromyces share many of the same peroxisomal marker genes, no copy of PEX3 has been found in Cardiosporidium/Nephromyces. PEX3 (along with PEX10, PEX12, and PEX19) is one of the 4 genes reportedly required for peroxisome function (Schluter et al. 2006). However, the fundamentals of peroxisome biology have been described from a limited set of eukaryotes, and organisms such as ciliates have peroxisomes but also lack PEX3 (Ludewig-Klingner et al. 2017). Therefore, PEX3 may not be critical to peroxisome function for alveolates, and possibly other under-studied eukaryotic lineages. Extreme sequence conservation of PEX3 and PEX19 is only found in opisthokonta and sequence divergence in other lineages may indicate alternative functions (Hua et al. 2015).

Two other genes (Sterol carrier protein 2, SCPX and Solute carrier family 27, member 2, VLACS) missing from Cardiosporidium/Nephromyces, but found in Toxoplasma, are involved in $\beta$-fatty acid oxidation. Both Cardiosporidium/Nephromyces encode the seven other $\beta$-fatty acid oxidation genes encoded in Toxoplasma, suggesting $\beta$-fatty acid oxidation forms part of the functional capabilities of the Cardiosporidium/Nephromyces peroxisome. Fatty acid oxidation is often a central component of peroxisome function and has been hypothesized to be the impetus for the evolution of peroxisomes (Speijer 2011). Based on transcript abundance, purine degradation in Nephromyces peroxisomes appears to be heavily utilized. Only $0.13 \%$ of genes had a higher 
transcription rate than urate oxidase in our data from wild collected

Nephromyces, and the other genes in the purine degradation pathway are among the most highly expressed transcripts in both wild and lab grown Nephromyces samples (Table 3). This result aligns with the previously reported high levels of urate oxidase protein in the renal sac of infected Molgula (Saffo 1988), indicating that the expression levels reported here do translate to protein. Much of this pathway is expressed over the $9^{\text {th }}$ percentile of all transcripts in Nephromyces, which corresponds to the top 100 genes. Expression of purine degradation genes in Cardiosporidium is far lower, and in the 70-90 percentile range (Table 3). Such high expression in Nephromyces represents an enormous metabolic investment and it is unlikely that these transcripts go largely untranslated.

Both Nephromyces and Molgula manhattensis encode xanthine dehydrogenase, and are able to convert xanthine into uric acid. Since we have identified the tunicate host as the source of purines, this raises the question of why Nephromyces is expressing xanthine dehydrogenase in the $97.87^{\text {th }}$ percentile, compared with similarly high tunicate expression (93.64th percentile). Although the percentile ranking between these two organisms cannot be directly compared, such high xanthine dehydrogenase expression in Nephromyces is surprising. It seems unlikely that so much xanthine dehydrogenase production is needed to convert only endogenous purines of Nephromyces. However, xanthine is only detected in the renal sac in small quantities, not nearly as abundant as uric acid, and xanthine dehydrogenase activity is restricted to the renal wall, not the 


\begin{tabular}{|c|c|c|c|c|c|c|c|c|c|c|}
\hline Gene & Wild Neph & $\begin{array}{c}\text { Lab grown } \\
\text { Neph } 1\end{array}$ & $\begin{array}{c}\text { Lab grown } \\
\text { Neph } 2\end{array}$ & $\begin{array}{c}\text { Cardio } \\
\text { Fraction } 1\end{array}$ & $\begin{array}{c}\text { Cardio } \\
\text { Fraction } 2\end{array}$ & $\begin{array}{c}\text { Cardio } \\
\text { Fraction } 3\end{array}$ & $\mathrm{Mm}$ & $\begin{array}{l}\text { Uninfected } \\
\text { Mm } 1\end{array}$ & $\begin{array}{l}\text { Uninfected } \\
\text { Mm } 2\end{array}$ & $\begin{array}{c}\text { Uninfected } \\
\mathrm{Mm} 3\end{array}$ \\
\hline xanthine dehydrogenase & 97.87 & 93.17 & 94.83 & none & 76.88 & 69.5 & 93.64 & N/A & $\mathrm{N} / \mathrm{A}$ & $\mathrm{N} / \mathrm{A}$ \\
\hline urate oxidase & 99.87 & 99.44 & 99.54 & 86.75 & 87.24 & 70.98 & - & - & - & - \\
\hline 5-hydroxyisourate hydrolase & 99.16 & 91.31 & 88.41 & 87.67 & 83.27 & 79.1 & - & - & - & - \\
\hline OHCU decarboxylase & 93.38 & - & - & - & - & - & - & - & - & - \\
\hline allantoinase & 99.09 & 98.38 & 98.23 & 73.61 & 90.32 & 71.89 & - & - & - & - \\
\hline amindohydrolase & 99.75 & 79.25 & 89.18 & 87.43 & 92.27 & 92.08 & - & - & - & - \\
\hline malate synthase & 59.17 & 93.81 & 93.11 & - & - & - & - & - & - & - \\
\hline serine-pyruvate transaminase & 99.85 & 99.57 & 99.79 & 84.64 & 80.81 & 77.79 & 85.65 & 91.17 & 71.85 & 75.05 \\
\hline
\end{tabular}

Table 3. Expression percentile ranking of purine degradation genes, from total expressed transcripts in Nephromyces (Neph), Cardiosporidium (Cardio) and Molgula (Mm). The wild Nephromyces and Molgula manhattensis data originate from the same RNA extraction and were bioinformatically separated. Data was also generated from laboratory grown tunicates, artificially infected with Nephromyces (Lab grown Neph 1 \& 2).

Cardiosporidium fractions represent 1) unfiltered pericardial fluid, 2) the $25 \%$ and 3) $30 \%$ fractions extracted from a sucrose gradient, and may contain different proportions of Cardiosporidium life stages. The three uninfected Molgula manhattensis were raised from gametes in the lab and never exposed to Nephromyces infection. The (-) denotes the transcript was not recovered in that dataset whereas (N/A) indicates the transcript was assembled, but the transcripts per million (TPM) was $<1$. 
renal lumen (Nolfi 1970). One possible explanation is that Nephromyces exports its xanthine dehydrogenase into the renal wall in order to drive the production of xanthine from hypoxanthine before the purine salvage enzymes adenine phosphoribosyltransferase and hypoxanthine-guanine phosphoribosyltransferase can salvage hypoxanthine into adenine and guanine. High expression of purine degradation genes in Nephromyces is clear, but the purpose is uncertain. It does indicate purine degradation is an important pathway for Nephromyces, however, the functional significance is not immediately obvious. Pathway analysis predicts that Nephromyces is able to convert xanthine into urea and ureidoglycolate, however neither compound is biologically useful without further conversion. We propose that the products of purine degradation in Nephromyces are converted to glyoxylate.

One possible route is the conversion of ureidoglycolate into glyoxylate. There are two known enzymes able to catalyze this conversion: ureidoglycolate lyase, found in fungi and bacteria, which catalyzes (s)-ureidoglycolate to glyoxylate and urea, and ureidoglycolate amidohydrolase, found in plants and bacteria, which catalyzes (s)-ureidoglycolate to glyoxylate, carbon dioxide, and ammonia (Muñoz et al. 2006; Percudani et al. 2013; Wells \& Lees 1991; Werner et al. 2010; Shin et al. 2012; Serventi et al. 2010). Both ureidoglycolate lyase and ureidoglycolate amidohydrolase are amidohydrolases - hydrolases that use amide bonds as substrates. No orthologs to either ureidoglycolate lyase and 
ureidoglycolate amidohydrolase have been found in the Nephromyces transcriptome. However, an amidohydrolase is present, which is predicted to be structurally similar to the ureidoglycolate amidohydrolase found in Arabidopsis, including similar location and number of zinc binding domains. This amidohydrolase also has a similarly high expression level as the other purine degradation enzymes (Table 3). In order to determine if the amidohydrolase found in Nephromyces is capable of catalyzing (s)-ureidoglycolate, functional assays will need to be performed.

While the functionality of this particular amidohydrolase has yet to be determined, its ability to act on an (s)-ureidoglycolate is an attractive hypothesis for a few reasons. One, there are two known enzymes capable of breaking the amide bond in (s)-ureidoglycolate that have independently evolved: ureidoglycolate lyase and ureidoglycolate amidohydrolase. This pathway has not been widely explored across eukaryotes and the modification to a class of molecules able to break amide bonds to accommodate the structure of (s)ureidoglycolate may not be a complex evolutionary step. Two, (s)-ureidoglycolate is unstable and will spontaneously convert to glyoxylate, albeit without the stereospecific conversion present when catalyzed by ureidoglycolate amidohydrolase (Werner et al. 2010). Spontaneous conversion of glyoxylate results in a 50\% loss of efficiency versus enzymatic conversion, presumably creating strong evolutionary pressure to enzymatically degrade (s)ureidoglycolate to maintain stereochemistry. 
Glyoxylate is a common substrate for a number of enzymes including glyoxylate oxidase, which catalyzes glyoxylate with water and oxygen to form oxalate and hydrogen peroxide (Kasai et al. 1963). Notably, no copy of glyoxylate oxidase has been identified in Nephromyces, which is surprising given that another common component of the renal sac is calcium oxalate (Saffo \& Lowenstam 1978). We have not identified any genes suggesting that Nephromyces or its bacterial endosymbionts can produce or process oxalate. Calcium oxalate is also found in uninfected hosts indicating that the tunicate is the source. Another enzyme that uses glyoxylate as a substrate, which is present in Nephromyces/Cardiosporidium, is serine-pyruvate transaminase (AGXT), which can be localized to peroxisomes or mitochondria, and catalyzes glyoxylate to glycine and pyruvate (Takada \& Noguchi 1985). An alternative enzyme for processing glyoxylate is malate synthase (MLS), which is also targeted to the peroxisome and missing from apicomplexans, including Cardiosporidium, but is found in Nephromyces (Figure 1).

Malate synthase is one of two genes integral to the glyoxylate cycle, an alternative pathway for part of the citrate cycle. In the glyoxylate cycle, isocitrate is converted into glyoxylate and succinate by isocitrate lyase (McFadden \& Howes 1965). Glyoxylate is combined with acetyl-CoA to create malate (Molina et al. 1994). This cycle allows for the creation of glucose from fatty acids directly (Kornberg \& Krebs 1957). The presence of malate synthase indicates at least a piece of the glyoxylate cycle is present in Nephromyces. No copy of isocitrate lyase 
is predicted from the Nephromyces transcriptome, and only a small fragment of a possible isocitrate synthase has been identified in Cardiosporidium. However, under the model proposed here, the generation of glyoxylate is from uric acid, and isocitrate synthase would not be required.

Both AGXT and MLS (in Nephromyces) show similarly high expression as the purine degradation genes (Table 3), which is consistent with our proposed uric acid to glyoxylate pathway. In particular, AGXT is among the most highly expressed Nephromyces transcripts, with consistently higher expression than MLS, possibly indicating it is the primary route of glyoxylate conversion. The products of AGXT, glycine and pyruvate, are versatile substrates and used by a number of pathways. Glycine is the simplest amino acid and an essential component of many important biological compounds, as a nitrogen source in a readily useable form. Pyruvate is extremely versatile and involved in several critical biological pathways. A non-inclusive list includes amino acid biosynthesis, acetyl-CoA biosynthesis, fatty acid biosynthesis, and the citric acid cycle. These pathways represent both carbon and energy acquisition (Figure 2). Additionally, Nephromyces has the ability to use MLS to convert glyoxylate and acetyl-CoA into malate, a compound central to the citric acid cycle, allowing for another mechanism of carbon and energy acquisition (Figure 2).

The hypothesized conversion of uric acid to glyoxylate in Nephromyces creates several possibilities. First, it allows for the metabolic waste product, uric acid, to be converted into glycine, pyruvate, and malate (Figure 2). Second, it 


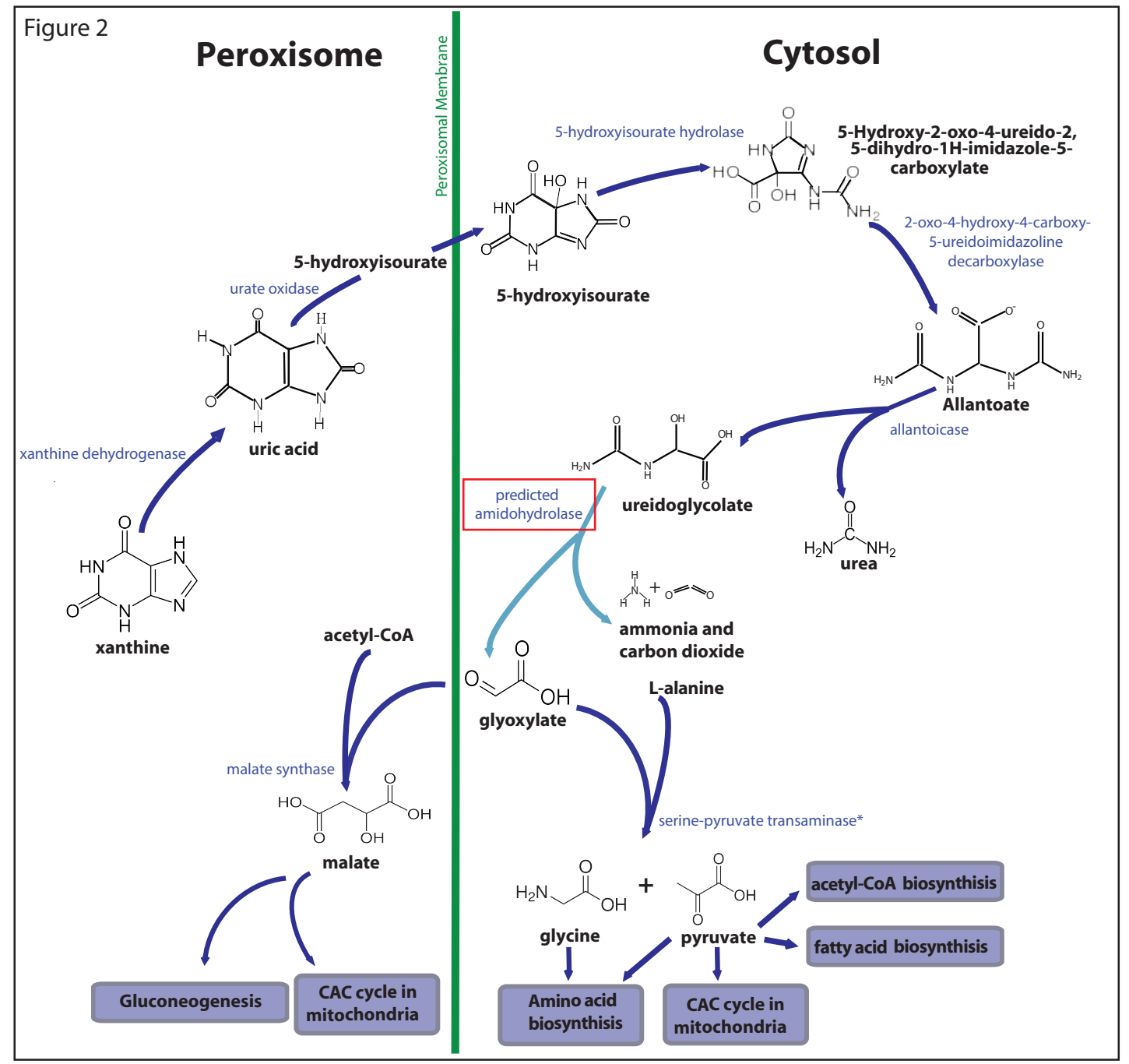

Figure 2. Predicted purine degradation pathway in Nephromyces, within the peroxisome and cytosol. Dark blue arrows represent enzymes identified in the Nephromyces transcriptome. The light blue arrow represents the highly expressed amidohydrolase (red box) predicted to convert ureidoglycolate into glyoxylate. Enzymes on the left side are localized to peroxisomes, the right side to the cytosol, with the green vertical line representing the peroxisomal membrane. The predicted pathway is able to convert uric acid into glyoxylate, and subsequent conversion by serine-pyruvate transaminase (AGXT) or malate synthase, creates glycine and pyruvate or malate respectively. The * by AGXT indicates ambiguous predicted localization, to either peroxisomes or mitochondria 
provides an explanation for the exceptionally high expression of the purine degradation pathway. Third, it gives Nephromyces access to a primary carbon, nitrogen, and an energy source at no cost to its host. And finally, this change in primary carbon, nitrogen, and energy could conceivably reduce the impact of Nephromyces on its host, allowing Nephromyces densities to increase while decreasing virulence. Reduction in virulence would have been a necessary first step toward mutualism.

Uric acid as a primary carbon and energy source is not completely unknown. Bacterial species have been found in chicken hutches that were able to grow solely on uric acid (Rouf \& Lomprey 1968; Thong-On et al. 2012), and some species of fungi are able to grow on media solely containing uric acid (Middelhoven et al. 1989). However, this is a novel substrate for an apicomplexan to grow on, and while it is unlikely that Nephromyces could survive on uric acid alone, it is a promising base for both carbon and nitrogen acquisition. It is possible that the Nephromyces bacterial endosymbionts (Sabree et al. 2009; Potrikus \& Breznak 1980) are contributing to the proposed purine to glucose pathway, but that is not currently supported by our data.

As the adaptive significance of uric acid deposits in tunicates, and particularly in Molgula, are unknown, it is difficult to speculate on the effects of Nephromyces uric acid degradation to the host. If these renal sac deposits are a form of excretion by storage, as has been hypothesized (Goodbody 1965), then 
having a symbiont that is capable of digesting uric acid may be beneficial simply by digesting an indigestible metabolite and converting uric acid into urea. Alternatively, once the uric acid has been broken down, the tunicate may benefit from metabolites derived from uric acid previously unavailable to the tunicate. If Nephromyces is overexpressing xanthine dehydrogenase in order to outcompete adenine phosphoribosyltransferase and hypoxanthine-guanine phosphoribosyltransferase, diverting hypoxanthine from purine salvage to purine degradation, there could be a potential cost to the host under purinelimited conditions.

Our data demonstrate that both the proposed mutualistic Nephromyces and parasitic Cardiosporidium encode the genes for purine degradation, which have been lost in other apicomplexans sequenced to date. Additionally, these genes share a common ancestry with chromerid genes, indicating they are not the product of a recent horizontal gene transfer from bacteria. These data also add support to the growing body of evidence that indicate the presence of peroxisomes in apicomplexans. Nephromyces and Cardiosporidium are predicted to have peroxisomes and, unlike any other apicomplexan, are capable of preforming both purine degradation and part of the glyoxylate cycle. The presence of purine degradation, AGXT, and MLS allow for the intriguing possibility of conversion of uric acid into a primary nitrogen, carbon and energy source. This predicted metabolic activity would be a completely novel substrate for an apicomplexan and may have been an important factor in the reduction of 
virulence in Nephromyces. 


\section{Acknowledgements}

Support for this project was provided by National Institute of Health, grant AI124092, as well as National Science Foundation award 1541510. CHS is supported by NSERC (Discovery grant RGPIN/05754-2015). 
Anderson TJC et al. 2000. Microsatellite Markers Reveal a Spectrum of Population Structures in the Malaria Parasite Plasmodium falciparum. Mol Biol Evol.

17:1467-1482. doi: 10.1093/oxfordjournals.molbev.a026247.

Arnott A, Barry AE, Reeder JC. 2012. Understanding the population genetics of Plasmodium vivax is essential for malaria control and elimination. Malar. J. 11:14. doi: 10.1186/1475-2875-11-14.

Arrowood MJ, Sterling CR. 2016. Isolation of Cryptosporidium Oocysts and Sporozoites Using Discontinuous Sucrose and Isopycnic Percoll Gradients Author ( s ): Michael J . Arrowood and Charles R. Sterling Published by : Allen Press on behalf of The American Society of Parasitologists St. J. Parasitol. 73:314-319.

Bankevich A et al. 2012. SPAdes: A New Genome Assembly Algorithm and Its Applications to Single-Cell Sequencing. J. Comput. Biol. 19:455-477. doi:

10.1089/cmb.2012.0021.

Bennett GM, Moran NA. 2013. Small, smaller, smallest: The origins and evolution of ancient dual symbioses in a phloem-feeding insect. Genome Biol. Evol. 5:16751688. doi: $10.1093 /$ gbe/evt118.

Bernsel A, Viklund H, Hennerdal A, Elofsson A. 2009. TOPCONS: Consensus prediction of membrane protein topology. Nucleic Acids Res. 37:465-468. doi: 10.1093/nar/gkp363.

Bolger A, Lohse M, Usadel B. 2014. Trimmomatic: A flexible trimmer for Illumina 
sequence data. Bioinformatics. 30:2114.

Boratyn GM, Thierry-Mieg J, Thierry-Mieg D, Busby B, Madden TL. 2018. MagicBLAST, an accurate DNA and RNA-seq aligner for long and short reads. bioRxiv. 390013. doi: 10.1101/390013.

Brayton KA et al. 2007. Genome sequence of Babesia bovis and comparative analysis of apicomplexan hemoprotozoa. PLoS Pathog. 3:1401-1413. doi: 10.1371/journal.ppat.0030148.

Brown AM V. et al. 2018. Comparative Genomics of Wolbachia-Cardinium Dual Endosymbiosis in a Plant-Parasitic Nematode. Front. Microbiol. 9:1-21. doi: 10.3389/fmicb.2018.02482.

Brunner JL, Collins JP. 2009. Testing assumptions of the trade-off theory of the evolution of parasite virulence. Evol. Ecol. Res. 11:1169-1188.

Bushnell B. 2014. BBMap: A Fast, Accurate, Splice-Aware Aligner.

Callahan BJ et al. 2016. DADA2 : High-resolution sample inference from Illumina amplicon data. Nat. Methods. 1-7. doi: 10.1038/nmeth.3869.

Cardoso R, Soares H, Hemphill A, Leitão A. 2016. Apicomplexans pulling the strings: manipulation of the host cell cytoskeleton dynamics. Parasitology. 1-14. doi: 10.1017/S0031182016000524.

Ciancio A et al. 2008. Redescription of Cardiosporidium cionae (Van Gaver and Stephan, 1907) (Apicomplexa: Piroplasmida), a plasmodial parasite of ascidian haemocytes. Eur. J. Protistol. 44:181-196. doi: 10.1016/j.ejop.2007.11.005. 
Cusa E et al. 1999. Genetic Analysis of a Chromosomal Region Containing Genes Required for Assimilation of Allantoin Nitrogen and Linked Glyoxylate Metabolism in Escherichia coli Genetic Analysis of a Chromosomal Region Containing Genes Required for Assimilation of Allantoin. 181:7479-7484.

Ding M, Clayton C, Soldati D. 2000. Toxoplasma gondii catalase: are there peroxisomes in toxoplasma? J. Cell Sci. 113 ( Pt 1:2409-2419.

Dong LC et al. 2006. First report on histology and ultrastructure of an intrahemocytic paramyxean parasite (IPP) from tunicate Halocynthia roretzi in Korea. Dis. Aquat. Organ. 72:65-69. doi: 10.3354/dao072065.

Emanuelsson O, Brunak S, von Heijne G, Nielsen H. 2007. Locating proteins in the cell using TargetP, SignalP and related tools. Nat. Protoc. 2:953-971. doi:

10.1038/nprot.2007.131.

English AC et al. 2012. Mind the Gap: Upgrading Genomes with Pacific Biosciences RS Long-Read Sequencing Technology. PLoS One. 7:1-12. doi: 10.1371/journal.pone.0047768.

Escalante a a, Ayala FJ. 1995. Evolutionary origin of Plasmodium and other Apicomplexa based on rRNA genes. Proc. Natl. Acad. Sci. U. S. A. 92:5793-7. doi: 10.1073/pnas.92.13.5793.

Finn RD et al. 2017. InterPro in 2017-beyond protein family and domain annotations. Nucleic Acids Res. 45:D190-D199. doi: 10.1093/nar/gkw1107.

Frank S a. 1996. Host-symbiont conflict over the mixing of symbiotic lineages. 
Proc. Biol. Sci. 263:339-344. doi: 10.1098/rspb.1996.0052.

Frank S a. 1996. Models of parasite virulence. Q. Rev. Biol. 71:37-78. doi:

$10.1086 / 419267$.

Frank SA. 1992. A kin selection model for the evolution of virulence. Proc R Soc L.

B. 250:195-197.

Gabaldon T. 2010. Peroxisome diversity and evolution. Philos. Trans. R. Soc. B Biol. Sci. 365:765-773. doi: 10.1098/rstb.2009.0240.

Gabaldon T, Ginger ML, Michels PAM. 2016. Peroxisomes in parasitic protists. Mol. Biochem. Parasitol. 209:33-45. doi: 10.1016/j.molbiopara.2016.02.005.

Gardner MJ et al. 2002. Europe PMC Funders Group Genome sequence of the human malaria parasite Plasmodium falciparum. Nature. 419:3-9. doi:

10.1038/nature01097.Genome.

Giard MA. 1888. On Nephromyces, a new genus of Fungi parasitic in the kidney of the Molgulidæ. Ann. Mag. Nat. Hist. Ser. 6. 1:386-388. doi:

$10.1080 / 00222938809460753$.

Goodbody BYI. 1965. Nitrogen Excretion in Ascidiacea. Enzyme. 34:299-305.

Greganova E, Steinmann M, Mäser P, Fankhauser N. 2013. In silico ionomics segregates parasitic from free-living eukaryotes. Genome Biol. Evol. 5:19021909. doi: $10.1093 /$ gbe/evt134.

Gruwell ME, Hardy NB, Gullan PJ, Dittmar K. 2010. Evolutionary relationships 
among primary endosymbionts of the mealybug subfamily phenacoccinae (Hemiptera: Coccoidea: Pseudococcidae). Appl. Environ. Microbiol. 76:75217525. doi: 10.1128/AEM.01354-10.

Guillou L et al. 2013. The Protist Ribosomal Reference database (PR2): A catalog of unicellular eukaryote Small Sub-Unit rRNA sequences with curated taxonomy. Nucleic Acids Res. 41:597-604. doi: 10.1093/nar/gks1160.

Haas BJ et al. 2014. reference generation and analysis with Trinity. doi: 10.1038/nprot.2013.084.De.

Holt C, Yandell M. 2011. MAKER2: an annotation pipeline and genome-database management tool for second-generation genome projects. BMC Bioinformatics. 12:491. doi: 10.1186/1471-2105-12-491.

Hua R, Gidda SK, Aranovich A, Mullen RT, Kim PK. 2015. Multiple Domains in PEX16 Mediate Its Trafficking and Recruitment of Peroxisomal Proteins to the ER. Traffic. 16:832-852. doi: 10.1111/tra.12292.

Hyde JE. 2007. Targeting purine and pyrimidine metabolism in human apicomplexan parasites. Curr. Drug Targets. 8:31-47. doi: $10.2174 / 138945007779315524$.

Jackman SD et al. 2017. ABySS 2 . 0 : resource-efficient assembly of large genomes using a Bloom filter. Genome Res. 27:768-777. doi:

10.1101/gr.214346.116.Freely.

Jackson AP. 2015. The evolution of parasite genomes and the origins of 
parasitism. Parasitology. 142:S1-S5. doi: 10.1017/S0031182014001516.

Janouškovec J et al. 2015. Factors mediating plastid dependency and the origins of parasitism in apicomplexans and their close relatives. Proc. Natl. Acad. Sci. U. S. A. 112:10200-7. doi: 10.1073/pnas.1423790112.

Janouskovec J, Keeling PJ. 2016. Evolution: Causality and the origin of parasitism. Curr. Biol. 26:R174-R177. doi: 10.1016/j.cub.2015.12.057.

Jung D-K et al. 2006. Structural and functional analysis of PucM, a hydrolase in the ureide pathway and a member of the transthyretin-related protein family. Proc. Natl. Acad. Sci. 103:9790-9795. doi: 10.1073/pnas.0600523103.

Kaasch AJ, Joiner KA. 2000. Targeting and Subcellular Localization of. J. Biol. Chem. 275:1112-1118.

Kada S, Lion S. 2015. Superinfection and the coevolution of parasite virulence and host recovery. J. Evol. Biol. 28:2285-2299. doi: 10.1111/jeb.12753.

Kahn K, Tipton PA. 1998. Spectroscopic characterization of intermediates in the urate oxidase reaction. Biochemistry. 37:11651-11659. doi: 10.1021/bi980446g.

Kanehisa M, Sato Y, Kawashima M, Furumichi M, Tanabe M. 2016. KEGG as a reference resource for gene and protein annotation. Nucleic Acids Res. 44:D457D462. doi: 10.1093/nar/gkv1070.

Kappmeyer LS et al. 2012. Comparative genomic analysis and phylogenetic position of Theileria equi. BMC Genomics. 13:603. doi: 10.1186/1471-2164-13603. 
Kasai T, Suzuki I, Asai T. 1963. Glyoxylate Oxidation in Acetobacter with Refenence to the formation of Oxalic Acid. Applyed Microbiol. 9:49-58. http://www.pubmedcentral.nih.gov/articlerender.fcgi?artid=1152716\&tool=pm centrez\&rendertype=abstract.

Katoh K, Standley DM. 2013. MAFFT multiple sequence alignment software version 7: Improvements in performance and usability. Mol. Biol. Evol. 30:772780. doi: $10.1093 / \mathrm{molbev} / \mathrm{mst} 010$.

Keeling PJ. 2004. Reduction and compaction in the genome of the apicomplexan parasite Cryptosporidium parvum. Dev. Cell. 6:614-616. doi: 10.1016/S15345807(04)00135-2.

Kemp LE, Yamamoto M, Soldati-Favre D. 2013. Subversion of host cellular functions by the apicomplexan parasites. FEMS Microbiol. Rev. 37:607-631. doi: 10.1111/1574-6976.12013.

Kleiner M et al. 2018. Metaproteomics method to determine carbon sources and assimilation pathways of species in microbial communities. Proc. Natl. Acad. Sci. 115:E5576-E5584. doi: 10.1073/pnas.1722325115.

Klindworth A et al. 2013. Evaluation of general 16S ribosomal RNA gene PCR primers for classical and next-generation sequencing-based diversity studies. Nucleic Acids Res. 41:1-11. doi: 10.1093/nar/gks808.

Koren S et al. 2014. Canu: scalable and accurate long-read assembly via adaptive k-mer weighting and repeat separation. Genome Res. 1-11. doi: 
10.1101/gr.215087.116.Freely.

Kornberg HL, Krebs HA. 1957. Synthesis of cell constituents from C2-units by a modified tricarboxylic acid cycle. Nature. 179:988-991. doi: 10.1038/179988a0.

Kumagai A et al. 2011. Soft tunic syndrome in the edible ascidian Halocynthia roretzi is caused by a kinetoplastid protist. Dis. Aquat. Organ. 95:153-161. doi: 10.3354/dao02372.

Kuwahara H et al. 2007. Reduced Genome of the Thioautotrophic Intracellular Symbiont in a Deep-Sea Clam, Calyptogena okutanii. Curr. Biol. 17:881-886. doi: 10.1016/j.cub.2007.04.039.

Laczny CC et al. 2015. VizBin - An application for reference-independent visualization and human-augmented binning of metagenomic data. Microbiome. 3:1-7. doi: 10.1186/s40168-014-0066-1.

Lalremruata A et al. 2017. Species and genotype diversity of Plasmodium in malaria patients from Gabon analysed by next generation sequencing. Malar. J. 16:1-11. doi: 10.1186/s12936-017-2044-0.

Lambert CC, Lambert G, Crundwell G, Kantardjieff K. 1998. Uric acid accumulation in the solitary ascidian Corella inflata. J. Exp. Zool. 282:323-331. doi:

10.1002/(SICI)1097-010X(19981015)282:3<323::AID-JEZ5>3.0.CO;2-0.

Langmead B, Salzberg SL. 2012. Fast gapped-read alignment with Bowtie 2. Nat. Methods. 9:357. http://dx.doi.org/10.1038/nmeth.1923.

Lee KS et al. 2011. Plasmodium knowlesi: Reservoir hosts and tracking the 
emergence in humans and macaques. PLoS Pathog. 7. doi:

10.1371/journal.ppat.1002015.

Leung TLF, Poulin R. 2008. Parasitism, commensalism, and mutualism: Exploring the many shades of symbioses. Vie Milieu. 58:107-115.

Li J et al. 1997. Regulation and trafficking of three distinct $18 \mathrm{~S}$ ribosomal RNAs during development of the malaria parasite. J. Mol. Biol. 269:203-213. doi: 10.1006/jmbi.1997.1038.

Li W, Godzik A. 2006. Cd-hit: A fast program for clustering and comparing large sets of protein or nucleotide sequences. Bioinformatics. 22:1658-1659. doi: 10.1093/bioinformatics/btl158.

Lige B, Jayabalasingham B, Zhang H, Pypaert M, Coppens I. 2009. 'Role of an Ancestral D-Bifunctional Protein Containing' 'Two Sterol-Carrier Protein-2 Domains in Lipid Uptake and Trafficking in Toxoplasma'. Mol. Biol. Cell. 20:658672.

Lopez-Sanchez MJ et al. 2009. Evolutionary convergence and nitrogen metabolism in Blattabacterium strain Bge, primary endosymbiont of the cockroach Blattella germanica. PLoS Genet. 5. doi:

10.1371/journal.pgen.1000721.

Ludewig-Klingner A-K, Michael V, Jarek M, Brinkmann H, Petersen J. 2018.

Distribution and Evolution of Peroxisomes in Alveolates (Apicomplexa, Dinoflagellates, Ciliates). Genome Biol. Evol. 10:1-13. doi: 10.1093/gbe/evx250. 
Mahler BYHR, The W, Assistamze T, Germille OF. 1955. Studies On Uricase. J. Biol. Chem.

Marin B, Nowack ECM, Melkonian M. 2005. A plastid in the making: Evidence for a second primary endosymbiosis. Protist. 156:425-432. doi:

10.1016/j.protis.2005.09.001.

Mccutcheon JP, Moran NA. 2011. in symbiotic bacteria. Nat. Publ. Gr. 10:13-26. doi: $10.1038 /$ nrmicro2670.

McCutcheon JP, Moran NA. 2007. Parallel genomic evolution and metabolic interdependence in an ancient symbiosis. Proc. Natl. Acad. Sci. U. S. A. 104:19392-19397. doi: 10.1073/pnas.0708855104.

McFadden B, Howes W. 1965. Crystallization and Some Properties of Isocitrate Lyase from Pseudomonas indigofera*. J. Biochem. 58:116-122.

Middelhoven WJ, De Hoog GS, Notermans S. 1989. Carbon assimilation and extracellular antigens of some yeast-like fungi. Antonie Van Leeuwenhoek. 55:165-175. doi: 10.1007/BF00404756.

Molina I, Pellicer M, Badia J, Aguilar J, Baldoma L. 1994. Molecular

Characterization of Escherichia coli Malate Synthase G: Differentiation with the Malate Synthase A Isoenzyme. Eur. J. Biochem. 224:541-548. doi:

10.1111/j.1432-1033.1994.00541.x.

Moog D, Przyborski JM, Maier UG. 2017. Genomic and proteomic evidence for the presence of a peroxisome in the apicomplexan parasite Toxoplasma gondii and 
other Coccidia. Genome Biol. Evol. 1-39. doi: 10.1093/gbe/evx231/4596564.

Moore RB et al. 2008. A photosynthetic alveolate closely related to apicomplexan parasites. Nature. 452:900-900. doi: 10.1038/nature06871.

Moran NA. 1996. Accelerated evolution and Muller's rachet in endosymbiotic bacteria. Proc. Natl. Acad. Sci. 93:2873-2878. doi: 10.1073/pnas.93.7.2873.

Moran NA, Dunbar HE, Wilcox JL. 2005. Regulation of transcription in a reduced bacterial genome: Nutrient-provisioning genes of the obligate symbiont Buchnera aphidicola. J. Bacteriol. 187:4229-4237. doi: 10.1128/JB.187.12.4229-4237.2005. Moran NA, McCutcheon JP, Nakabachi A. 2008. Genomics and Evolution of Heritable Bacterial Symbionts. Annu. Rev. Genet. 42:165-190. doi: 10.1146/annurev.genet.41.110306.130119.

Moriya Y, Itoh M, Okuda S, Yoshizawa AC, Kanehisa M. 2007. KAAS: An automatic genome annotation and pathway reconstruction server. Nucleic Acids Res. 35:182-185. doi: 10.1093/nar/gkm321.

Morrison HG et al. 2007. Genomic Minimalism in the Early Diverging Intestinal Parasite Giardia lamblia. Science (80-. ). 317:1921-1926. doi:

10.1126/science.1143837.

Muñoz A, Raso MJ, Pineda M, Piedras P. 2006. Degradation of ureidoglycolate in French bean (Phaseolus vulgaris) is catalysed by a ubiquitous ureidoglycolate urea-lyase. Planta. 224:175-184. doi: 10.1007/s00425-005-0186-8.

Mushegian AA, Ebert D. 2016. Rethinking 'mutualism' in diverse host-symbiont 
communities. BioEssays. 38:100-108. doi: 10.1002/bies.201500074.

Nakaia K, Horton P. 1999. PSORT : a program for detecting sorting signals in proteins and predicting their subcellular localization. Comput. CORNER. 0004:1336-1337.

Nishino T, Okamoto K, Eger BT, Pai EF, Nishino T. 2008. Mammalian xanthine oxidoreductase - Mechanism of transition from xanthine dehydrogenase to xanthine oxidase. FEBS J. 275:3278-3289. doi: 10.1111/j.17424658.2008.06489.x.

Nolfi JR. 1970. Biosynthesis of uric acid in the tunicate, Molgula manhattensis, with a general scheme for the function of stored purines in animals. Comp. Biochem. Physiol. 35:827-842. doi: 10.1016/0010-406X(70)90078-2.

Nowack ECM, Melkonian M. 2010. Endosymbiotic associations within protists. Philos. Trans. R. Soc. Lond. B. Biol. Sci. 365:699-712. doi:

10.1098/rstb.2009.0188.

Ogedengbe ME, Qvarnstrom Y, da Silva AJ, Arrowood MJ, Barta JR. 2015. A linear mitochondrial genome of Cyclospora cayetanensis (Eimeriidae, Eucoccidiorida, Coccidiasina, Apicomplexa) suggests the ancestral start position within mitochondrial genomes of eimeriid coccidia. Int. J. Parasitol. 45:361-365. doi: 10.1016/j.ijpara.2015.02.006.

Percudani R, Carnevali D, Puggioni V. 2013. Ureidoglycolate hydrolase, amidohydrolase, lyase: How errors in biological databases are incorporated in 
scientific papers and vice versa. Database. 2013:1-9. doi:

10.1093/database/bat071.

Petersen G, Cuenca A, Møller IM, Seberg 0. 2015. Massive gene loss in mistletoe (Viscum viscaceae) mitochondria. Sci. Rep. 5:1-7. doi: 10.1038/srep17588.

Philip LA, Tullman-Ercek, George G. 2006. NIH Public Access. Annu. Rev. Microbiol. 373-395. doi: 10.1146/annurev.micro.60.080805.142212.The.

Potrikus CJ, Breznak JA. 1980. Uric acid-degrading bacteria in guts of termites (Reticulitermes flavipes (Kollar)). Appl. Environ. Microbiol. 40:117-124. doi: 16345587.

Quinlan AR, Hall IM. 2010. BEDTools: A flexible suite of utilities for comparing genomic features. Bioinformatics. 26:841-842. doi:

10.1093/bioinformatics/btq033.

Rao Q et al. 2015. Genome reduction and potential metabolic complementation of the dual endosymbionts in the whitefly Bemisia tabaci. BMC Genomics. 16:1-13. doi: 10.1186/s12864-015-1379-6.

Reid AJ et al. 2012. Comparative genomics of the apicomplexan parasites Toxoplasma gondii and neospora caninum: Coccidia differing in host range and transmission strategy. PLoS Pathog. 8. doi: 10.1371/journal.ppat.1002567.

Reid AJ. 2014. Large, rapidly evolving gene families are at the forefront of hostparasite interactions in Apicomplexa. Parasitology. 1-14. doi: 10.1017/S0031182014001528. 
Roos DS. 2005. Themes and Variations in Apicomplexan Parasite Biology. Science (80-. ). 309:72-73. doi: 10.1126/science.1115252.

Rouf MA, Lomprey RF. 1968. Degradation of uric acid by certain aerobic bacteria. J. Bacteriol. 96:617-622.

Sabree ZL, Kambhampati S, Moran N a. 2009. Nitrogen recycling and nutritional provisioning by Blattabacterium, the cockroach endosymbiont. Proc. Natl. Acad. Sci. U. S. A. 106:19521-19526. doi: 10.1073/pnas.0907504106.

Saffo M. 1990. Symbiosis within a symbiosis: Intracellular bacteria within the endosymbiotic protist Nephromyces. Mar. Biol. 107:291-296. doi:

10.1007/BF01319828.

Saffo M, McCoy A, Rieken C, Slamovits C. 2010. Nephromyces, a beneficial apicomplexan symbiont in marine animals. Proc. Natl. Acad. Sci. U. S. A. 107:16190-16195. doi: 10.1073/pnas.1002335107.

Saffo MB. 1982. DISTRIBUTION OF THE ENDOSYMBIONT NEPHROMYCES GIARD WITHIN THE ASCIDIAN FAMILY MOLGULIDAE. Biol Bull. 162:95-104.

Saffo MB. 1988. Nitrogen Waste or Nitrogen Source? Urate Degradation in the Renal Sac of Molgulid Tunicates. Biol. Bull. 175:403. doi: 10.2307/1541732.

Saffo MB, Davis WL. 1982. Modes of Infection of the Ascidian Molgula manhattensis by Its Endosymbiont Nephromyces Giard. Biol. Bull. 162:105. doi: $10.2307 / 1540974$.

Saffo MB, Lowenstam H a. 1978. Calcareous deposits in the renal sac of a molgulid 
tunicate. Science. 200:1166-1168. doi: 10.1126/science.200.4346.1166.

Sakharkar KR, Kumar Dhar P, Chow VVTK. 2004. Genome reduction in prokaryotic obligatory intracellular parasites of humans: A comparative analysis. Int. J. Syst. Evol. Microbiol. 54:1937-1941. doi: 10.1099/ijs.0.63090-0.

Schluter A et al. 2006. The evolutionary origin of peroxisomes: An ER-peroxisome connection. Mol. Biol. Evol. 23:838-845. doi: 10.1093/molbev/msj103.

Schlüter A et al. 2006. The evolutionary origin of peroxisomes: An ER-peroxisome connection. Mol. Biol. Evol. 23:838-845. doi: 10.1093/molbev/msj103.

Schlüter A, Real-Chicharro A, Gabaldón T, Sánchez-Jiménez F, Pujol A. 2009.

PeroxisomeDB 2.0: An integrative view of the global peroxisomal metabolome. Nucleic Acids Res. 38:800-805. doi: 10.1093/nar/gkp935.

Schultz AC, Nygaard P, Saxild HH. 2001. Functional analysis of 14 genes that constitute the purine catabolic pathway in Bacillus subtilis and evidence for a novel regulon controlled by the PucR transcription activator. J. Bacteriol. 183:3293-3302. doi: 10.1128/JB.183.11.3293-3302.2001.

Seah B, Saffo MB, Cavanaugh CM. 2011. A Tripartite Animal-Protist-Bacteria Symbiosis: Culture-Independent and Phylogenetic Characterization. Harvard.

Seemann T. 2014. Prokka: Rapid prokaryotic genome annotation. Bioinformatics. 30:2068-2069. doi: 10.1093/bioinformatics/btu153.

Serventi F et al. 2010. Chemical basis of nitrogen recovery through the ureide pathway: Formation and hydrolysis of S-ureidoglycine in plants and bacteria. ACS 
Chem. Biol. 5:203-214. doi: 10.1021/cb900248n.

Shin I, Percudani R, Rhee S. 2012. Structural and functional insights into (S)ureidoglycine aminohydrolase, keyenzyme of purine catabolism in Arabidopsis thaliana. J. Biol. Chem. 287:18796-18805. doi: 10.1074/jbc.M111.331819.

Simão FA, Waterhouse RM, Ioannidis P, Kriventseva E V., Zdobnov EM. 2015. BUSCO: Assessing genome assembly and annotation completeness with singlecopy orthologs. Bioinformatics. 31:3210-3212. doi:

10.1093/bioinformatics/btv351.

Small I, Peeters N, Legeai F, Lurin C. 2004. Predotar: A tool for rapidly screening proteomes for N-terminal targeting sequences. Proteomics. 4:1581-1590. doi: 10.1002/pmic.200300776.

De Souza DJ, Bézier A, Depoix D, Drezen JM, Lenoir A. 2009. Blochmannia endosymbionts improve colony growth and immune defence in the ant Camponotus fellah. BMC Microbiol. 9:1-8. doi: 10.1186/1471-2180-9-29. Speijer D. 2011. Oxygen radicals shaping evolution: Why fatty acid catabolism leads to peroxisomes while neurons do without it: FADH2/NADH flux ratios determining mitochondrial radical formation were crucial for the eukaryotic invention of peroxisomes and catabolic tissue. BioEssays. 33:88-94. doi: 10.1002/bies.201000097.

Stamatakis A. 2014. RAxML version 8: A tool for phylogenetic analysis and postanalysis of large phylogenies. Bioinformatics. 30:1312-1313. doi: 
10.1093/bioinformatics/btu033.

Stanke M, Steinkamp R, Waack S, Morgenstern B. 2004. AUGUSTUS: A web server for gene finding in eukaryotes. Nucleic Acids Res. 32:309-312. doi:

10.1093/nar/gkh379.

Takada Y, Noguchi T. 1985. Characteristics of alanine: glyoxylate aminotransferase from Saccharomyces cerevisiae, a regulatory enzyme in the glyoxylate pathway of glycine and serine biosynthesis from tricarboxylic acidcycle intermediates. Biochem. J. 231:157-63. doi: 10.1042/bj2310157.

Thong-On A et al. 2012. Isolation and Characterization of Anaerobic Bacteria for Symbiotic Recycling of Uric Acid Nitrogen in the Gut of Various Termites. Microbes Environ. 27:186-192. doi: 10.1264/jsme2.ME11325.

Urakawa $\mathrm{H}$ et al. 2005. Hydrothermal vent gastropods from the same family (Provannidae) harbour E- and y -proteobacterial endosymbionts. Environ. Microbiol. 7:750-754. doi: 10.1111/j.1462-2920.2005.00753.x.

Usuda $\mathrm{N}$ et al. 1994. Uric acid degrading enzymes, urate oxidase and allantoinase, are associated with different subcellular organelles in frog liver and kidney. J. Cell Sci. 107 ( Pt 4:1073-81. http://www.ncbi.nlm.nih.gov/pubmed/8056832.

Wang J, Wang Y, Gao C, Jiang L, Guo D. 2017. PPero, a computational model for plant PTS1 type peroxisomal protein prediction. PLoS One. 12:1-12. doi: 10.1371/journal.pone.0168912.

Wells XE, Lees EM. 1991. Ureidoglycolate amidohydrolase from developing 
french bean fruits (Phaseolus vulgaris [L.].). Arch. Biochem. Biophys. 287:151159. doi: 10.1016/0003-9861(91)90400-D.

Wernegreen JJ. 2015. Endosymbiont evolution: Predictions from theory and surprises from genomes. Ann. N. Y. Acad. Sci. 1360:16-35. doi:

10.1111/nyas.12740.

Wernegreen JJ. 2017. In it for the long haul: evolutionary consequences of persistent endosymbiosis. Curr. Opin. Genet. Dev. 47:83-90. doi:

10.1016/j.gde.2017.08.006.

Werner AK, Romeis T, Witte CP. 2010. Ureide catabolism in Arabidopsis thaliana and Escherichia coli. Nat. Chem. Biol. 6:19-21. doi: 10.1038/nchembio.265.

Wickham H. 2016. ggplot2: Elegant Graphics for Data Analysis. Springer-Verlag New York http://ggplot2.org.

Woo YH et al. 2015. Chromerid genomes reveal the evolutionary path from photosynthetic algae to obligate intracellular parasites. Elife. 4:1-41. doi: 10.7554/eLife.06974.

Xi H, Schneider BL, Reitzer L. 2000. Purine catabolism in Escherichia coli and function of xanthine dehydrogenase in purine salvage. J. Bacteriol. 182:53325341. doi: 10.1128/JB.182.19.5332-5341.2000.

Zarowiecki M, Berriman M. 2015. What helminth genomes have taught us about parasite evolution. Parasitology. 142 Suppl:S85-97. doi:

10.1017/S0031182014001449. 


\section{Chapter 3}

Comparative transcriptomics of two apicomplexans (Nephromyces and Cardiosporidium cionae) and the genomes of Nephromyces bacterial endosymbionts

by

Christopher Paight ${ }^{1}$, Elizabeth S. Hunter ${ }^{1}$, Claudio H. Slamovits ${ }^{2}$, Mary Beth Saffo $^{1,3}$ \& Christopher E Lane ${ }^{1 *}$

will be submitted to the journal Proceedings of the National Academy of Sciences

${ }^{1}$ Department of Biological Sciences, University of Rhode Island, Kingston RI, 02881, USA.

2 Department of Biochemistry and Molecular Biology, Dalhousie University, Halifax, Canada

${ }^{3}$ Smithsonian National Museum of Natural History, Washington, DC 20560, USA. 


\section{CHAPTER 3}

\section{Abstract}

Parasitism has been referred to as an "evolutionary dead end", because the transition to parasitism is unidirectional. Once an organism becomes an obligate parasite, it is likely to remain an obligate parasite due to the loss of metabolic pathways. Nephromyces is a genus in the parasitic phylum Apicomplexa, but has an apparent mutualistic relationship with Molgula tunicates. Support for a mutualistic relationship is based largely on a nearly $100 \%$ host infection rate with no known clearance of Nephromyces. Because transition away from obligate parasite is so rare, little is known about the evolutionary steps involved in such a transition - particularly in lineages with such a long history of obligate parasitism as Apicomplexa. In order to examine this unusual evolutionary transition, we sequenced transcriptomes from Nephromyces and its parasitic sister taxon, Cardiosporidium cionae, which is an excellent model for what Nephromyces might have looked like as a parasite. Both C. ciona and Nephromyces have tunicate hosts and bacterial endosymbionts, but each maintains a different lifestyle. A comparison of Nephromyces, C. ciona, and their endosymbionts will be presented with a focus on system dynamics, relationships, and clues to how this transition occurred.

\section{Introduction}

In 2010, Saffo et al. characterized the apicomplexan Nephromyces as 
having a mutualistic relationship with its host Molgula tunicates. The significance of a mutualistic genus within a group of $>6000$ obligate parasites, some of which cause massive human mortality, prompted us to initiate a genomic investigation of Nephromyces. The unique evolutionary pressures caused by an obligate parasitic lifestyle, namely host immune system evasion and the ready abundance of pre-formed host metabolites, lead to predictable patterns of gene family losses and expansions. Typically this consists of the expansion of gene families related to host immune system evasion and other parasitism related functions, and the subsequent loss of many of the core biosynthetic genes due to their presence in the intracellular environment [1-5]. Due to these pressures, obligate parasitism is often an evolutionary dead end.

Besides Nephromyces, the phylum Apicomplexa is composed entirely of obligate metazoan parasites. As a result of an estimated 800 million years of evolution as obligate parasites, [6], many of the genomic patterns associated with parasitism have been described from the apicomplexan lineages. Gene expansions can be seen in the plasmodium var protein family, which are involved in host manipulation, evasion and in the expansion of rhoptry, microneme, and dense granule proteins. The list of core biosynthetic pathways lost in apicomplexans includes purine biosynthesis, purine degradation, biosynthesis of many amino acids, and vitamin biosynthesis. These losses make the parasite dependent on the host, not only for primary carbon and nitrogen, but also for any metabolites it can no longer generate by either de novo synthesis or by 
conversion. High demand on the host for these metabolites to fuel parasite growth increases the cost of infection, thereby increasing virulence. Parasites must maintain a delicate balance between transmission, virulence, and host immune system evasion.

The trade offs in this balance have been described in detail [7-11], but one common solution many parasites adopt is maintaining low relative abundance inside the host. Higher parasite abundance will increase the cost to that host, and increase virulence. If the parasites kill the host before completing their lifecycle or before transmission to a new host, their fitness falls to zero. Similarly, if parasites have a high prevalence in a population and high lethality, they risk decimating their host population. High-sustained infection prevalence is a good indicator of low virulence, and low virulence is often achieved by self-limited reproduction by the parasites. In this way, Nephromyces stood out as a very atypical parasite. Nephromyces has a nearly $100 \%$ infection rate, sustained almost year-round. Unexpectedly, based on typical host / parasite dynamics, Nephromyces also reaches very high cell densities. These atypical epidemiological factors were the basis for the Saffo et al. 2010 conclusion that Nephromyces must be mutualistic. In order to reach high cell densities while maintaining low virulence, Nephromyces was predicted to produce something of high value to the host, to offset the cost associated with maintaining such high densities of an obligate parasite.

The unusual epidemiology of Nephromyces becomes more apparent when 
contrasted with its parasitic sister taxon, Cardiosporidium cionae.

Cardiosporidium, first described in 1907 by Van Gaver and Stephan, and later described by Ciancio et al 2008 is a blood parasite found in solitary nonmolgulidae ascidian hosts, including Ciona intestinalis. Cardiosporidium quickly reaches and maintains $\sim 95 \%$ infection prevalence by late July. In contrast to Nephromyces, Cardiosporidium cell densities remain low, with orders of magnitude difference in cell densities (based on DNA extraction quantities as a proxy for cell density). Virulence in both of these apicomplexans is thought to be low based on histological work by Ciancio et al. 2008 and Saffo and Nelson 1982. Low virulence is also predicted from the high-sustained infection prevalence. The contrast in cell densities between Nephromyces and Cardiosporidium, along with the lack of apparent virulence, indicates an unusual relationship between Nephromyces and its host.

In addition to being sister taxa, Nephromyces and Cardiosporidium share a number of other traits. Both organisms are monoxenous, with ascidians as the only host, both have infective stages that are transmitted through seawater, localize within the pericardium of the host and each harbor a monophyletic bacterial endosymbiont species that has been maintained since Nephromyces and Cardiosporidium diverged (Figure 3). These similarities make Cardiosporidium an ideal organism to compare with Nephromyces in order to resolve the genomic changes taking place behind the transition from obligate parasitism to a mutualistic host-symbiont relationship. 
However, there are some key differences, besides the epidemiological factors, between Nephromyces and Cardiosporidium, including host species. Cardiosporidium infects several genera of tunicates including Ciona, Halocynthia, Styela, Ascidiella and possibly others [12,13], while Nephromyces is restricted to the Molgulidae family of tunicates [14]. Interestingly, Cardiosporidium has not been found in any Molgulidae tunicates. Another key difference is that Cardiosporidium is an intracellular blood parasite, while Nephromyces is extracellular (another unusual trait for an apicomplexan). Additionally, Nephromyces is exclusively found in a Molgulidae specific structure called the renal sac, a large ductless structure of unknown function $[15,16]$.

Despite its name, the renal sac does not seem to function as a typical renal organ, but was named for the large deposits of crystallized uric acid and calcium oxalate within it. Many ascidians have localized deposits of uric acid, but tunicates in the Molgulidae family have the largest $[17,18]$. While the function of these deposits in the tunicate remain unclear, previous work demonstrated that Nephromyces is able to degrade uric acid because it retains the ancestral purine degradation genes lost in all other apicomplexans (Chapter 2). Transcriptome data and pathway analysis suggest that uric acid may be the primary source of carbon and nitrogen for Nephromyces (Chapter 2). Uric acid is an atypical source of carbon and nitrogen, but it is not unheard of. There are several species of bacteria and fungi which can be cultured on media containing only uric acid [1921]. Due to their unusual environment inside the renal sac and because 
Nephromyces is extracellular, this organism may not have access to all the required pre-formed metabolites. However, the genus Nephromyces is reported to maintain three different bacterial endosymbionts [22].

In addition to the monophyletic alphaproteobacteria, Nephromyces also harbors two other bacterial endosymbionts: a Betaproteobacteria and a Bacteroidetes. Acquisition and maintenance of bacterial endosymbionts is a common way for eukaryotes to gain new metabolic pathways and capabilities. The functional capabilities of bacterial endosymbionts exploited by eukaryotic hosts, for example include amino acid metabolism and vitamin metabolism [23], nitrogen metabolism [24], defense [25], chemotrophic energy production [26], and photosynthesis [27], to name a few. While bacterial endosymbionts are common in many protist lineages, they are rare in the phylum Apicomplexa. The only known apicomplexans to contain bacterial endosymbionts are Cardiosporidium and Nephromyces. This limited distribution to apicomplexans with ascidian hosts may be due to an unknown aspect of ascidian biology. Previous speculation that Nephromyces' bacterial endosymbionts are responsible for the observed high levels of purine degradation have recently been rejected (Chapter 2). However, the bacterial endosymbionts are likely instrumental to Nephromyces' ability to colonize the renal sac.

In order to examine the claim of mutualism, characterize the relationships involved in this tripartite endosymbiosis, and determine how Nephromyces achieves low virulence with high cell density, we sequenced the transcriptomes 
of Molgula manhattensis, Ciona intestinalis, Nephromyces, Cardiosporidium, and their bacterial endosymbionts. Additionally, to better understand the dynamics of the renal sac and the interplay between Nephromyces and its bacterial endosymbionts, we sequenced and partially assembled the Nephromyces genome and the genomes of all three types of the bacterial endosymbionts.

\section{Results}

\section{RNA Sequencing}

The contents of a single renal sac from an individual Molgula manhattensis yielded $32 \mathrm{ng} / \mu \mathrm{l}$ RNA by Qubit and resulted in 195,694 transcripts from $M$. manhattensis, Nephromyces, and the bacterial endosymbionts. After binning by species, 60,223 transcripts were attributed to Nephromyces. The cardiac fluid from 40 infected Ciona intestinalis individuals resulted in 109,446 transcripts, including 15,541 Cardiosporidium transcripts.

The large number of transcripts identified from Nephromyces was due to multiple species infection of a single host. Clustering sequences together resulted in 26938 transcripts at $90 \%, 23850$ at $80 \%, 21762$ at $70 \%, 19540$ at $60 \%, 16668$ at $50 \%$. Due to the multi species nature of Nephromyces infections, the transcriptome is a pan-genome assembly rather than a precise uni-species dataset, but we estimate that there are between 8000 and 12000 unique transcripts in Nephromyces. Kyoto Encyclopedia of Genes and Genomes (KEGG) functionally predicts 13336 transcripts in the full dataset. The tool BUSCO was 
used to assess the completeness of Nephromyces transcriptome resulting in $81.8 \%$ complete transcripts and $6.3 \%$ partial.

RNA extractions for Cardiosporidium samples yielded $164 \mathrm{ng} / \mu \mathrm{l}$ for nonsucrose gradient separated blood, $48 \mathrm{ng} / \mu \mathrm{l}$ from cells taken from the $25 \%$ layer, and $24 \mathrm{ng} / \mu \mathrm{l}$ from cells taken from the $30 \%$ layer. These resulted in 97,417,356 reads from the non-sucrose separated sample, 115,085,369 reads from cells at the $25 \%$ layer, and $116,393,114$ reads from the $30 \%$ layer. Separated by species, 3877 transcripts were from $C$. intestinalis, 16,663 transcripts were from Cardiosporidium, 1,689 transcripts were from the bacterial endosymbiont of Cardiosporidium. KEGG functionally predicts 9,775 total transcripts for Cardiosporidium, and BUSCO analysis for reports $69.7 \%$ complete and $11.9 \%$ partial coverage. BUSCO analysis for the bacterial endosymbiont transcriptome resulted in $14.8 \%$ complete and 19.6\% partial against bacterial_od9.

\section{Nephromyces genome}

The Nephromyces genome assembly remains highly fragmented and consists of 1176 contigs greater than $5 \mathrm{~kb}$ with a maximum length of $287,191 \mathrm{bp}$ and an average length of $36 \mathrm{~kb}$.

$\alpha$-proteobacteria genome (N $\alpha$ )

Two different alphaproteobacteria endosymbionts were recovered from our genomic data and assembled into a draft genome. The presence of two closely related alpha proteobacteria genomes both with high AT bias (25\% GC content) 
and regions of low complexity have limited our ability to assemble these genomes completely (Table 4). The two genomes assemble into 11 contigs ranging in size from $13 \mathrm{~kb}$ to $312 \mathrm{~kb}$ for a combined length of 995,540 and an average of 90,503. Based on transcriptome sequencing we estimate that the genome is largely complete. The draft genome contains 844 predicted coding sequences, 35 tRNAs matching all codons, and 4 rRNAs. Of the 546 predicted genes have KASS annotations (119 Genetic information processing, 32 Carbohydrate metabolism, 30 Energy metabolism, 29 Cellular processes, 26 Nucleotide metabolism, 26 Metabolism of cofactors and vitamins, 25 environmental information processing, 15 lipid metabolism, 12 amino acid metabolism, 15 unclassified).

Bacteroidetes Genome (Nbe)

Nephromyces bacteroidetes genome is circular, 494,352 nucleotides long, and extremely AT rich (22\% GC content) (Table 4). The genome contains 503 predicted genes, 31 tRNAs predicted to recognize all codons, and 4 rRNAs. 391 of the predicted genes have KASS annotations (110 Genetic information processing, 40 Carbohydrate metabolism, 38 Energy metabolism, 31 amino acid metabolism, 21 Metabolism of cofactors and vitamins, 17 Nucleotide metabolism, 11 unclassified, 10 Lipid metabolism, 9 Cellular processes).

Betaproteobacteria Genome (N $\beta \mathrm{e})$

The Betaproteobacteria genome is circular and 866,396 bp long with $30 \%$ GC content (Table 4). It contains 880 predicted genes, 40 tRNAs, and 4 rRNAs 


\begin{tabular}{|c|c|c|c|}
\cline { 2 - 4 } \multicolumn{1}{c|}{} & $\begin{array}{l}\text { Nephromyces's } \\
\text { Alphaproteobacterial } \\
\text { genome (Nae) }\end{array}$ & $\begin{array}{l}\text { Nephromyces's } \\
\text { Bacteroidetes } \\
\text { Genome (NBe) }\end{array}$ & $\begin{array}{l}\text { Nephromyces's } \\
\text { Betaproteobacterial } \\
\text { Genome (Nße) }\end{array}$ \\
\hline contigs & 11 & 1 & 1 \\
\hline size in BP & 995,540 & 494,352 & 866,396 \\
\hline CDS & 844 & 503 & 880 \\
\hline tRNA & 35 & 31 & 40 \\
\hline rRNA & 4 & 4 & 4 \\
\hline GC content & $25 \%$ & $22 \%$ & $30 \%$ \\
\hline
\end{tabular}

Table 4) Genomic assembly statistics for Nephromyces three bacterial endosymbionts. N $\alpha \mathrm{e}$ and Nbe were assembled from a hybrid assembly of Illumina HiSeq and Pacific Biosciences data, while $\mathrm{N} \beta \mathrm{e}$ was assembled with Pacific Biosciences. Sequence data contained multiple closely related strains and these assemblies represent pangenomes of the various symbionts. 
(two identical 16s copies and 2 identical 23s copies). 753 of the 880 predicted genes have KAAS annotations (156 Genetic information processing, 61 Carbohydrate metabolism, 47 Energy metabolism, 11 Cellular processes, 45 Nucleotide metabolism, 68 Metabolism of cofactors and vitamins, 39 environmental information processing, 18 lipid metabolism, 62 amino acid metabolism, 14 unclassified).

\section{Bacterial Genome BUSCO Analysis}

BUSCO analysis against bacteria_odb9 for the alphaproteobacteria endosymbionts resulted in 99 complete BUSCO copies, 97 single copy and 2 duplicate, and 5 fragmented copies, and is predicted to be $66.9 \%$ complete and 3.4\% incomplete. For the bacteroidetes endosymbiont BUSCO returned 88 complete single copy, no duplicates, and 10 fragmented for a 59.5\% complete and 6.8\% incomplete. The Betaproteobacteria endosymbiont had 116 complete BUSCO copies, all single copy (78.4\%), 15 incomplete (10.1\%), and 17 missing $(11.5 \%)$.

Nephromyces and Cardiosporidium metabolic pathway characterization

\section{Endocytosis}

Both Nephromyces/Cardiosporidium are predicted to encode genes for clathrin-dependant endocytosis. An additional 25 genes related to endocytosis 
are predicted in Nephromyces/Cardiosporidium than in P. falciparum. Many of the additional genes found in Nephromyces/Cardiosporidium are in the VPS and CHMP protein families and form part of the ESCRT machinery important in the biogenesis of multivesicular bodies. Multivesicular bodies transport ubiquitinated proteins to lysosomes for degradation. Other proteins not found in P. falciparum include a number of genes in the AP2 complex, which are accessory proteins in clathrin-mediated endocytosis. The AP2 complex plays an important role in the regulation of the assembly of clathrin-coated vesicles.

Carbohydrate Metabolism

Basic carbon metabolism in Nephromyces/Cardiosporidium is similar to other apicomplexans and encodes the complete pathways for the citrate acid cycle, glycolysis, gluconeogenesis, and the pentose phosphate pathway. Interestingly, Nephromyces/Cardiosporidium are predicted to encode far more genes involved with inositol phosphate metabolism than either P. falciparum or T. gondii. Despite the absence of genes involved in the synthesis of myo-inositol in P. falciparum or T. gondii there is support that myo-inositol is used in intracellular calcium signaling in these two organisms. How $P$. falciparum or $T$. gondii are able to use myo-inositol without being able to synthesize it is unclear, but it is presumed that they have divergent and unrecognizable myo-inositol biosynthesis genes. However, both Nephromyces/Cardiosporidium are predicted to be able to synthesize myo-inositol and these genes are readily identifiable as orthologous to myo-inositol biosynthesis genes in other organisms. Both 
Nephromyces/Cardiosporidium have a copy of serine-pyruvate aminotransferase (AGXT), which catalyzes glyoxylate to glycine and pyruvate. Additionally, Nephromyces encodes malate synthase, which, in conjunction with acetyl-CoA, forms malate from glyoxylate.

Fatty Acid Metabolism

Nephromyces/Cardiosporidium are predicted to be able to perform fatty acid initiation and elongation in both the mitochondrial and cytoplasmic pathways, as well as elongation in the endoplasmic reticulum. Additionally, Nephromyces/Cardiosporidium are predicted to encode D-glycerate 3-kinase (GLYK), aldehyde dehydrogenase (ALDH), alcohol dehydrogenase (ADH), glycerol kinase (glpK), glycerol-3-phosphate 0-acyltransferase (GPAT1), and 1-acyl-snglycerol-3-phosphate acyltransferase (plsC), and thus are able create triglycerides from glucose.

Nucleic acid metabolism

Unique among apicomplexans, Nephromyces/Cardiosporidium contain a complete pathway for the biosynthesis of inosine monophosphate (IMP). IMP is a purine and the starting molecule for the biogenesis of guanine and adenine. De novo biosynthesis of purines has been lost in all sequenced apicomplexans. Other apicomplexans are capable of scavenging precursor molecules to IMP and converting them into IMP, but Nephromyces/Cardiosporidium encode the entire IMP biosynthesis pathway from 5-Phosphoribosyl diphosphate (PPRP). The genes involved in this pathway include amidophosphoribosyltransferase (purF), 
phosphoribosylamine---glycine ligase (purD), phosphoribosylformylglycinamidine synthase (purL), phosphoribosylformylglycinamidine cyclo-ligase (purM), phosphoribosylaminoimidazole carboxylase (PAICS), phosphoribosylaminoimidazole-succinocarboxamide synthase (purC), adenylosuccinate lyase (purB), IMP cyclohydrolase (purH). From IMP both Nephromyces/Cardiosporidium have the metabolic machinery for the biosynthesis of adenine and guanine. In addition to the biosynthesis of purines, Nephromyces/Cardiosporidium are also the only known apicomplexans capable of purine degradation (Chapter 2).

Biosynthesis of Amino Acids

Nephromyces is predicted to be able to synthesis 10 amino acids (alanine, asparagine, aspartic acid, cysteine, glutamine, glutamic acid, glycine, methionine, serine, and threonine). Cardiosporidium is predicted to be able to synthesis the same amino acids with the exception of cysteine. In addition to the complete pathways Nephromyces/Cardiosporidium have partial pathways for synthesis of phenylalanine from phenylpyruvate and can convert tyrosine from phenylalanine. Both Nephromyces/Cardiosporidium encode branched-chain amino acid aminotransferase, which adds the final amine group to valine, leucine, and isoleucine.

Vitamin and cofactor synthesis

Nephromyces has the predicted biosynthetic capabilities to produce 
riboflavin, acetyl CoA, nicotinate, folate, retinol, vitamin E, heme, and ubiquinone. Cardiosporidium has similar predicted biosynthetic capabilities, but is only capable of synthesizing 6-Geranylgeranyl-2,3-dimethylbenzene-1,4-diol and lacks the final two enzymes in the production of vitamin E. Both Nephromyces/Cardiosporidium encode a copy of lipoyl synthase (lipA), but lack lipoyl(octanoyl) transferase (lipB) in the lipoic acid synthesis pathway. Lipoic acid is an essential cofactor involved in the citric acid cycle, pyruvate dehydrogenase complex, 2-oxoglutarate dehydrogenase complex, branched-chain oxoacid dehydrogenase, and acetoin dehydrogenase.

\section{Endosymbiont pathways (Figure 3)}

Carbohydrate metabolism

The $\alpha$-proteobacteria endosymbiont of Nephromyces (N $\alpha$ e) has an extremely reduced carbohydrate metabolism, including all of the genes involved with gluconeogenesis and glycolysis. The only carbohydrate metabolism genes present are a complete citrate acid cycle and pyruvate dehydrogenase E1 component (aceE), dihydrolipoamide dehydrogenase (pdhD), and pyruvate dehydrogenase E2 component (aceF), which converts pyruvate to acetyl-CoA. With such severe reduction in carbohydrate metabolism, pyruvate appears to be the only carbon source the alphaproteobacteria is capable of processing.

The bacteroidetes endosymbiont (Nbe) has a similarly reduced carbohydrate metabolism as in the $\alpha$-proteobacteria, however the reduction is 


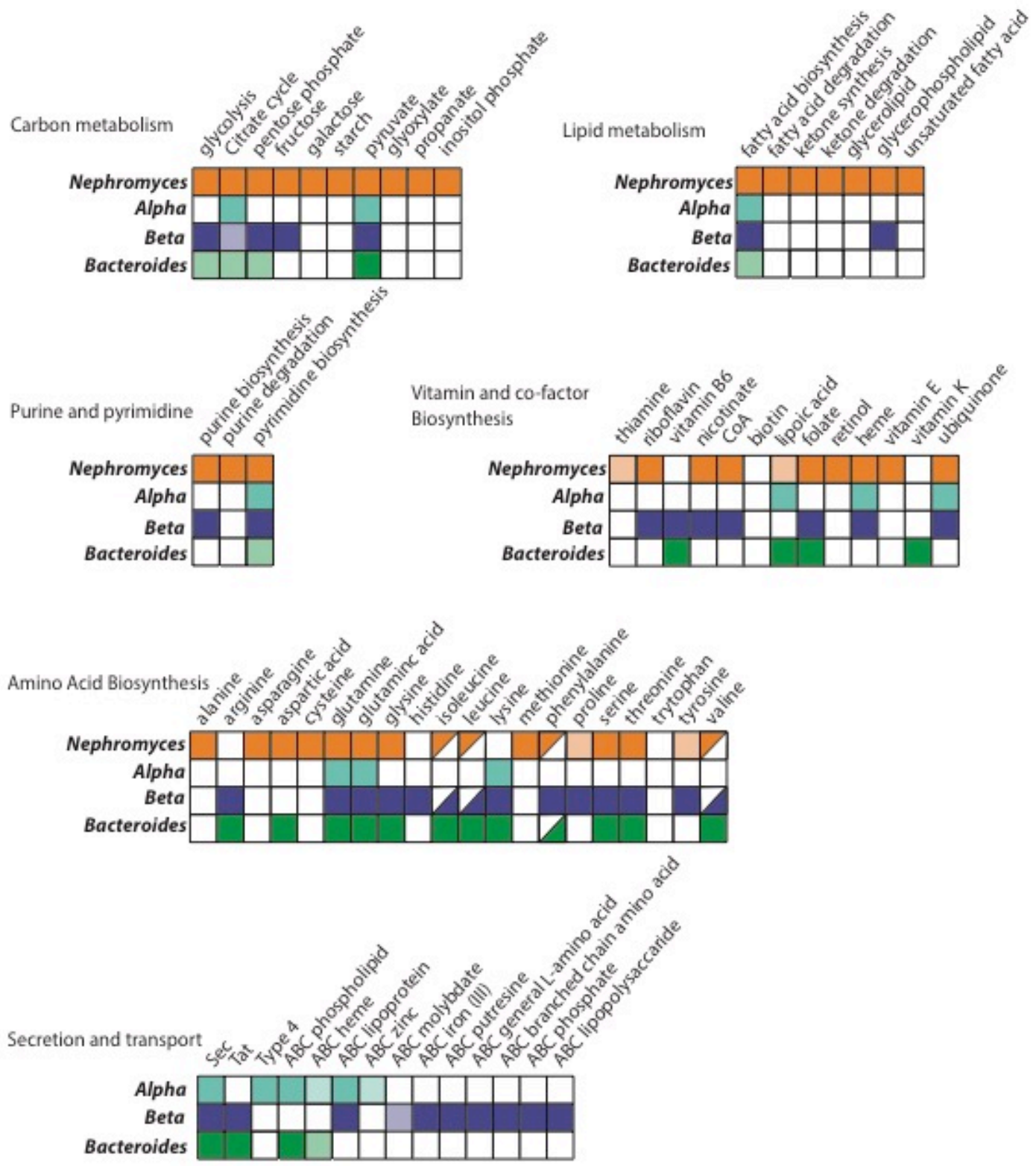

Figure 3) Metabolic pathway capabilities of Nephromyces (orange) and its bacterial endosymbionts (alpha=teal, beta=purple, bacteroidetes=green) solid colored boxes indicated a complete pathway, light shaded boxes indicated a partial pathway, and white boxes indicate the pathway is not present. 
not as extreme. Having lost gluconeogenesis the bacteroidetes endosymbiont can process fructose into phosphoenolpyruvate and contains pyruvate dehydrogenase E1 component (aceE), dihydrolipoamide dehydrogenase (pdhD), and pyruvate dehydrogenase E2 component (aceF) to convert pyruvate into acetyl-CoA. While the full citrate cycle is incomplete the partial cycle from 2oxoglutarate to oxaloacetate is complete, as well as the reductive pentose phosphate pathway from glyceraldehyde-3P to ribulose-5P.

Nephromyces $\beta$-proteobacteria endosymbiont (N $\beta \mathrm{e}$ ) has the most complete carbohydrate metabolism encoding the complete non-oxidative pentose phosphate pathway, the citrate cycle from 2-oxoglutarate to oxaloacetate, and the core glycolysis module involving three carbon compounds.

\section{Fatty Acid metabolism}

Paradoxically, while the enzymes involved with fatty acid biosynthesis

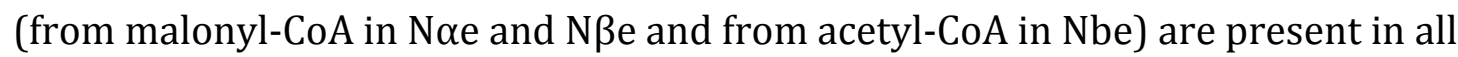
three types of bacterial endosymbiont, all the genes involved in fatty acid degradation have been lost in every symbiont. N $\alpha$ e and $\mathrm{N} \beta \mathrm{e}$ have a reduced glycerophospholipid metabolism and must convert phosphatidate to synthesis phosphatidylethanolamine, phosphatidylglycerol, and phosphatidylserine, while $\mathrm{N} \beta \mathrm{e}$ is able to de novo synthesize glycerophospholipids from glycerone. 
Nucleic acid metabolism

Purine metabolism is similarly reduced in both Noe and Nbe. Both endosymbionts lack all genes in the IMP biosynthesis pathway, as well as the ability to convert IMP to guanine or adenine. With so few purine biosynthesis capabilities, all of the guanine and adenine for DNA replication must be obtained as preformed nucleobases. $\mathrm{N} \beta \mathrm{e}$ purine biosynthesis is complete encoding adenylosuccinate lyase (purB), and IMP cyclohydrolase (purH) and is able to synthesize IMP from 5-Phosphoribosyl diphosphate (PRPP) (with the histidine synthesis pathway) as well as the genes required to convert IMP to both guanine and adenine. However, none of the three endosymbionts encode any genes involved in purine degradation.

Both Nae and $\mathrm{N} \beta \mathrm{e}$ encode the necessary genes for pyrimidine biosynthesis. However, because Nbe is missing several genes involved in pyrimidine biosynthesis it seems likely that Nbe is dependent on Nephromyces for both purines and pyrimidines

\section{Biosynthesis of Amino Acids}

Noe is only capable of synthesizing the amino acids glutamine, glutamic acid, and lysine. Of these three amino acids, lysine is the only amino acid that Nephromyces is unable to synthesize.

Nbe is capable of synthesizing 11 amino acids: arginine, aspartic acid, glutamine, glutamic acid, glycine, isoleucine, leucine, lysine, serine, threonine, and 
valine. Bacteroidetes is also able to synthesis phenylpyruvate, but lacks the ability to the ability to synthesis phenylalanine. Biosynthesis of arginine, isoleucine, leucine, lysine, and valine are not present in the Nephromyces transcriptome and may represent the bacteroidetes contribution. Additionally, Bacteroidetes synthesis of phenylpyruvate, but inability to synthesis phenylalanine compliments Nephromyces synthesis of phenylalanine from phenylpyruvate, but inability to synthesis phenylpyruvate.

$\mathrm{N} \beta \mathrm{e}$ encodes the genes for 11 amino acids: arginine, glutamine, glutamic acid, glycine, histidine, lysine, phenylalanine, proline, serine, threonine, and tyrosine. $\mathrm{N} \beta \mathrm{e}$ also encodes all the genes for synthesis of isoleucine, leucine, and valine, but lacks the last gene in the pathway branched-chain amino acid aminotransferase. However, Nephromyces encodes branched-chain amino acid aminotransferase and may be able to complete isoleucine, leucine, and valine by adding the final amine group.

\section{Vitamin and cofactor synthesis}

Nae only encodes genes for the biosynthesis three vitamins and cofactors; heme, ubiquinone, and lipoic acid. Lipoic acid has been experimentally shown to be exclusively synthesized in the apicoplast in apicomplexans; additionally all lipoic acid used in the mitochondria needs to be scavenged from the host. Lipoic acid may be an important product produced by the alphaproteobacteria. In contrast Nbe is capable of biosynthesis of vitamin B6, 
lipoic acid, folate and vitamin $\mathrm{K}$. N $\beta$ e is able to synthesize most cofactors including riboflavin, vitamin B6, nicotinate, coenzyme-A, folate, heme, and ubiquinone.

\section{Secretion and Transporters}

All three symbionts encode at least a partial bacterial sec secretion system. $\mathrm{N} \beta e$ is the most complete missing only secM. N $\alpha$ e lacks secE, secG, and secM. Nbe is the most incomplete missing secB, secD, secE, secM with so many genes missing it is not clear if the sec secretion system is functional in Nbe. Both $\mathrm{N} \beta \mathrm{e}$ and Nbe have the twin-arginine translocation pathway. N $\alpha$ e has TatC, but lacks TatA and is therefore incomplete [28]. While N $\alpha$ e is missing the Tat transport system, Nae does encode the type 4 bacterial secretion system.

In addition to the more general secretion systems there are also more specific $A B C$ transporters. Nbe has the fewest $A B C$ transporters only phospholipid and possibly a heme transporter. N $\alpha$ e also encodes a phospholipid and heme transporter in addition to a lipoprotein transporter and possibly a zinc transporter. $\mathrm{N} \beta \mathrm{e}$ 's genome contain the most $\mathrm{ABC}$ transporters, including Iron(III), putracine, General L-amino acid, branched-chain amino acid, phosphate, lipoprotein, lipopolysaccharide, and possibly a molybdate transporter.

\section{Bacterial phylogeny}

Nephromyces and Cardiosporidium alphaproteobacteria endosymbionts 
are monophyletic ( 98 bootstrap support) and are in the genus Rickettsia (94 bootstrap support). Nephromyces bacteroidetes endosymbiont is sister to the genus Pedobacter (87 bootstrap support) in the family Sphingobacteriaceae. The Betaproteobacteria fall within the genus Bordetella (98 bootstrap support) (Figure 4).

Orthology

Nephromyces had 21,762 genes when clustered at 70\% of these 20881 were assigned to orthogroups. $39.7 \%$ of orthogroups made contained Nephromyces and there were eight genus specific orthogroups containing 21 genes (Figure 5). 3,455 orthogroups were shared by Nephromyces, Apicomplexa, and Chromerids. 421 orthogroups were shared between Nephromyces and Apicomplexa (Figure 6). 218 orthogroups were shared between Nephromyces and Chromerids that were not found in Apicomplexa. Cardiosporidium had 7,395 genes, of which 6,977 were assigned to orthogroups. $31.5 \%$ of orthogroups contained Cardiosporidium and there was one species-specific orthogroup containing five genes. 2,778 orthogroups were shared between Cardiosporidium, Apicomplexa, and Chromerids. 236 orthogroups were shared between Cardiosporidium and Apicomplexa and not found in Chromerid. 219 orthogroups were shared between Cardiosporidium and Chromerids that were not found in Apicomplexa. Nephromyces and Cardiosporidium had 3, 106 shared orthogroups. Nephromyces had 1,178 orthogroups not found in Cardiosporidium. 289 


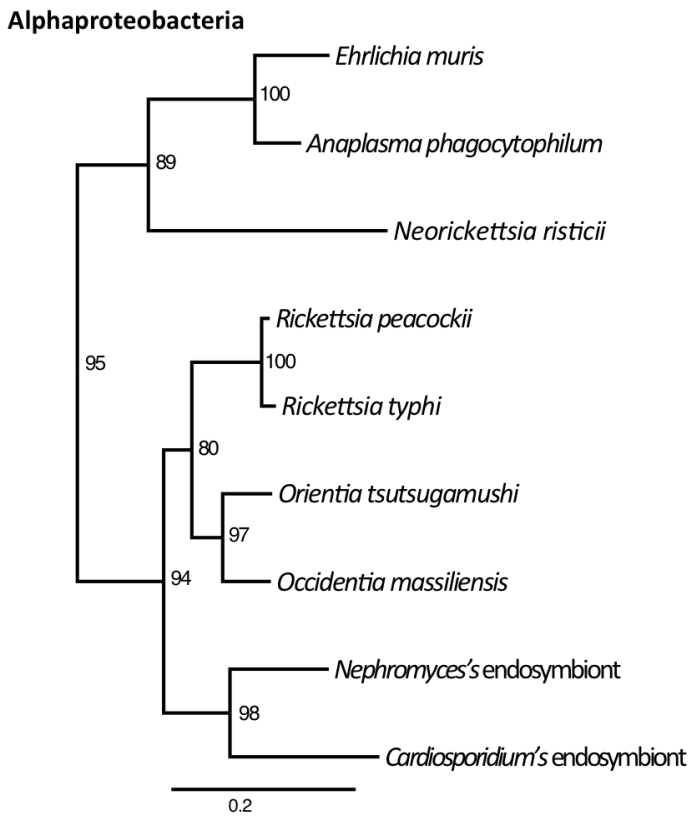

Bacteroidetes

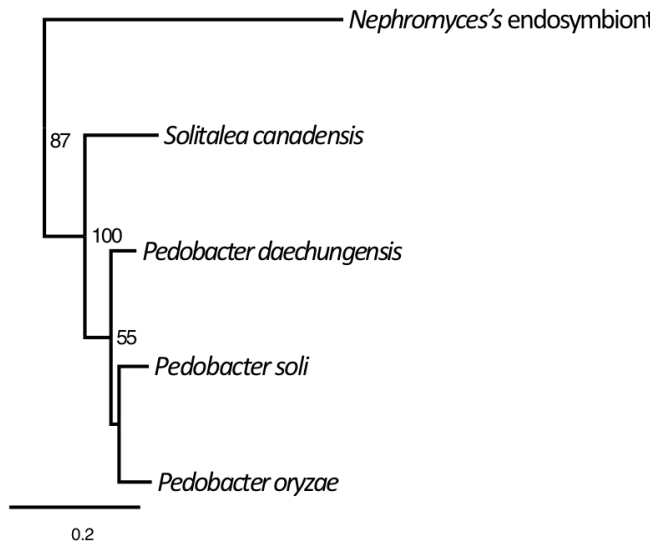

Betaproteobacteria



Figure 4) Subsets from larger maximum liklihood16s rRNA trees composed of Alphaproteobacteria, Bacteroidetes, and Betaproteobacteria sequences. Nodes are labeled with percentage bootstrap support values.

Nephromyces and Cardiosporidium alphaproteobacteria endosymbionts are monophyletic and sister to the family rickettsiaceae. Nephromyces bacteroidetes endosymbiont is in the family sphingobacteriaceae. Nephromyces betaproteobacteria endosymbiont is sister to the genus Bordetella. 


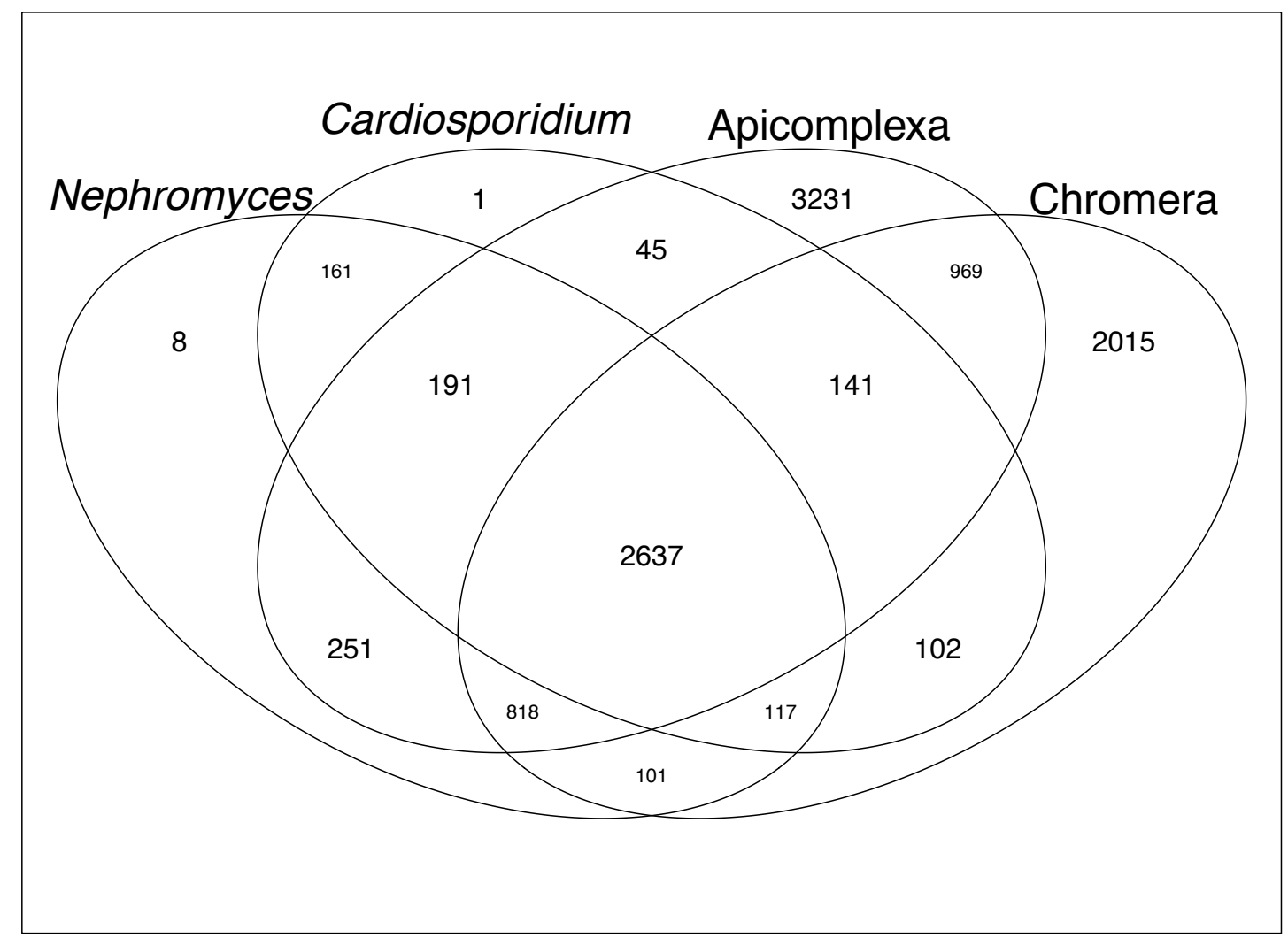

Figure 5) Collapsed Venn Diagram of orthologous gene groups from Nephromyces (70\% identity level), Cardiosporidium, Chromera ( $V$. brassicaformis, C. velia), and Apicomplexa (C. parvum, G. niphandrodes, B. bovis, T. parva, P. falciparum, C. cayetanensis, E. brunetti, E. falciformis, E. tenella, H. hammondi, N. caninum, S. neurona, T. gondii). Orthology was predicted with OrthoFinder. 


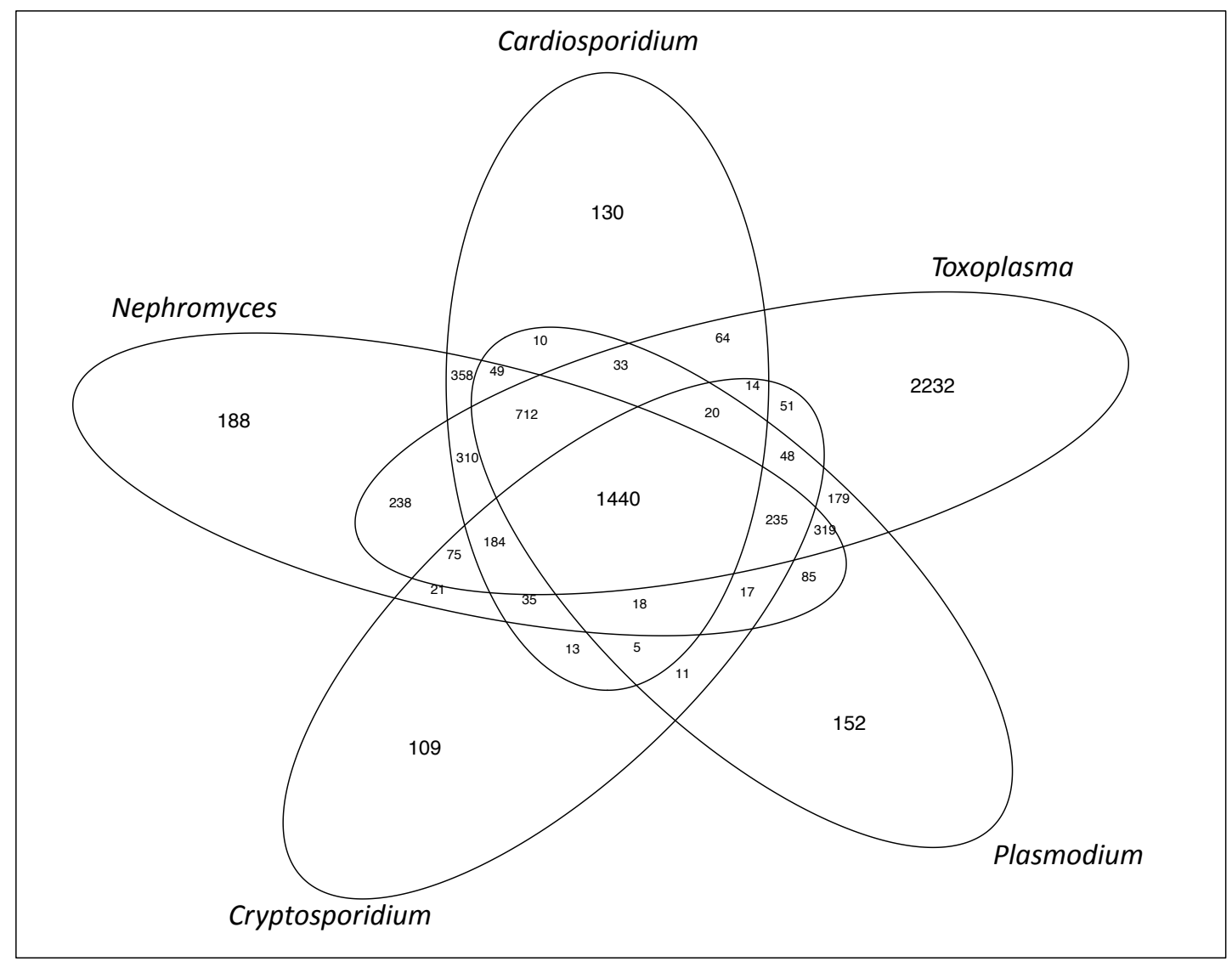

Figure 6) Venn Diagram of orthologous gene groups from Nephromyces (70\% identity level), Cardiosporidium, Toxoplasma, Cryptosporidium and Plasmodium. Orthology was predicted with OrthoFinder. 
orthogroups were found in Cardiosporidium and not in Nephromyces.

\section{Discussion}

Our initial aim in sequencing and comparing the transcriptomes of Nephromyces/Cardiosporidium was to better characterize mutualism in Nephromyces. A mutualistic relationship was described based on the unusual epidemiology Nephromyces, but high infection prevalence is not proof of mutual benefit. While our current efforts do not conclusively characterize Nephromyces' relation to its host, the data do provide insight into Nephromyces' atypical lifestyle. By comparing Nephromyces (a proposed mutualist) to Cardiosporidium (a blood parasite) we are able to extricate the evolutionary effects of a changing relationship from the evolutionary effects of an ascidian host.

Since all of the sequencing on Nephromyces has been on samples containing multiple Nephromyces species, our Nephromyces data is therefore a pan-transcriptome/genome. This approach limits our results and conclusions. This is particularly evident in our genomic assemblies, which are highly fragmented and almost certainly poly-species chimeric. In addition to problems with assembling the genomes of closely related organisms, we are also unable to estimate gene family expansions or reductions in Nephromyces. While these limitations are significant, the pan-transcriptome/genome does reflect the natural biology of Nephromyces. All of the renal sacs sampled from the host $M$. manhattensis to date have contained multiple Nephromyces infections. Efforts to 
culture single species isolates in the lab have met with limited success. This indicates that sustained Nephromyces infection is dependent on contributions from the community of species and endosymbionts.

Notably, by sequencing multiple Nephromyces species we were able to recover the transcriptomes/genomes of all three of Nephromyces endosymbionts. Recovering multiple bacterial endosymbiont types provides key insights into how this system works, and outweighs the disadvantages of a pantranscriptome/genome approach.

The transcriptomes for Nephromyces/Cardiosporidium are largely complete (estimated by BUSCO), but Cardiosporidium is estimated to be about $10 \%$ less complete than Nephromyces. We have taken this difference into account when comparing these two data sets. Both Nephromyces/Cardiosporidium encode an estimated eight thousand genes, which is a large number of genes for an apicomplexan. This estimate is similar to the number of genes in the most gene rich apicomplexan, Toxoplasma, which also encodes eight thousand genes. This is interesting given the phylogenetic placement of Nephromyces/Cardiosporidium in the hematozoa. Hematozoa, which contains plasmodiidae and piroplasmida lineages, have some of the smallest genomes with the least number of genes of any sequenced apicomplexans. This high gene number in both Nephromyces/Cardiosporidium may indicate that greater biosynthesis and metabolic capabilities are necessary for living in an ascidian host.

The two most striking observations made over the course of this 
exploration involve purine metabolism. The first is purine degradation; both Nephromyces/Cardiosporidium have the metabolic capabilities to convert xanthine into glyoxylate. Glyoxylate can then be converted, with serine-pyruvate aminotransferase (AGXT), into glycine and pyruvate; Nephromyces additionally encodes malate synthase (MLS), which combines Glyoxylate and acetyl-CoA, into malate. This is the proposed primary route of carbon, nitrogen, and energy acquisition for Nephromyces (Chapter 2). This pathway is absent in all other sequenced apicomplexans, but it appears the enzymes in this pathway were retained from the last common ancestor of Apicomplexa, and not a more recent horizontal gene transfer.

Nephromyces/Cardiosporidium also purine metabolism is de novo purine biosynthesis. While some apicomplexan lineages have one or two genes to synthesize inosine monophosphate (IMP) from immediate precursors, Nephromyces/Cardiosporidium are predicted to encode the entire de novo purine synthesis from 5-Phosphoribosyl diphosphate (PRPP). As the inability to synthesize purines has been widely targeted for drug development against other apicomplexan species, its presence in Nephromyces/Cardiosporidium is surprising [29].

The presence of both purine degradation and purine synthesis could be critical to Nephromyces' unusual epidemiology. By obtaining the bulk of the required carbon, nitrogen and energy from a metabolic waste product (i.e. tunicates lack the enzymatic ability to degrade purines past uric acid), 
Nephromyces is able to limit its impact on the host while still reaching high cellular densities. De novo synthesis of purines indicates that neither Nephromyces/Cardiosporidium is dependent of the host for IMP. In fact, these purine degradation and biosynthesis pathways may have been the integral factors that allowed Nephromyces to leave the intracellular environment and colonize the renal sac.

Another critical factor in Nephromyces' ability to survive in the renal sac is likely its bacterial endosymbionts. The $\alpha$-proteobacteria endosymbionts found in Nephromyces and Cardiosporidium are monophyletic, which indicates they have been maintained and vertically transmitted since the divergence of Nephromyces and Cardiosporidium. In addition to the $\alpha$-proteobacteria, Nephromyces has acquired a $\beta$-proteobacteria and a Bacteroidetes endosymbiont. The $\alpha$ proteobacteria and Bacteroidetes symbionts show a marked reduction in carbon metabolism with the N $\alpha$ e, only encoding genes for the citric acid cycle.

Bacteroidetes is only capable of processing three carbon compounds and encodes a partial citric acid cycle. Such pronounced reduction suggests that Nephromyces provides its symbionts a limited 'diet'. In both symbionts, carbon metabolism may be dependent on pyruvate, which is one of the products of AGXT. Related to this limited carbon metabolism, all three of the bacterial endosymbionts paradoxically encode complete fatty acid biosynthesis, but lack fatty acid degradation. Presumably, the fatty acid biosynthesis is for the construction of membranes, but without fatty acid degradation these symbionts are incapable of 


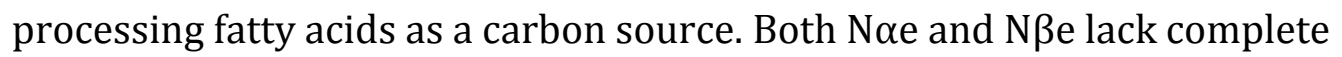
pathways for the creation of glycerophospholipids, and both contain phospholipid ABC transporters. This could indicate a dependence on Nephromyces for phospholipids.

None of the three endosymbionts of Nephromyces contain any genes involved in purine degradation. The absence of this entire pathway in the genomes of the endosymbionts is further support that the high levels of uric oxidase detected are from Nephromyces and not from any of its bacterial endosymbionts. Similarly, $\mathrm{N} \beta \mathrm{e}$ and N$\alpha \mathrm{e}$ do not encode any genes involved in de novo purine biosynthesis, including genes for the conversion from IMP to adenine and guanine. $\mathrm{N} \beta \mathrm{e}$ can likely synthesize purines from PPRP through the histidine biosynthesis pathway and contains the genes to synthesize adenine and guanine


these symbionts dependent on Nephromyces for both adenine and guanine. If Nephromyces were incapable of de novo purine biosynthesis then the entire renal sac community would be dependent on either the tunicate host for all purines, or on $\mathrm{N} \beta \mathrm{e}$. This would be a significant burden on the host and would markedly increase the cost of infection, which does not align with Nephromyces' strategy of low virulence and high-density infection of its host. This argument adds support to the prediction that Nephromyces/Cardiosporidium are able to synthesize purines.

Given the reduced genomes and correspondingly reduced metabolic 
capabilities, $\mathrm{N} \beta \mathrm{e}$ and Nbe encode a large proportionally high number of genes for synthesizing amino acids, vitamins, and co-factors. Together N $\beta$ e and Nbe could provide Nephromyces with all but one essential amino acid (tryptophan). N $\beta$ is predicted to synthesize leucine, isoleucine, and valine up to the last step, where the final amine group is added. Conversely, Nephromyces only encodes the last step in the conversion of these three amino acids. Similarly, Nbe encodes a partial biosynthetic pathway for phenylalanine, which seems to be complemented by Nephromyces. Nae is capable of synthesizing three amino acids and only one, which is an essential amino acid, is not encoded by Nephromyces (lysine). Vitamin and cofactor biosynthesis in N $\alpha$ e is also limited, synthesizing heme, ubiquinone, and lipoic acid, with lipoic acid being the only product Nephromyces may be incapable of synthesizing itself.

With such limited vitamin and amino acid metabolism encoded in the N $\alpha \mathrm{e}$ genome it is unlikely that Nephromyces is maintaining Nae just for lysine biosynthesis, but from the data we are unable to propose what particular function Nae serves Nephromyces. In addition, all of the limited species infections of Nephromyces we have been able to culture so far have an Nae type of symbiont. While neither the frequency of N $\alpha$ e or the limited species cultures are strong

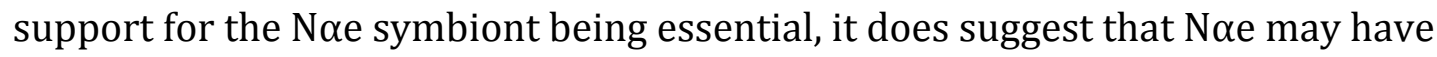
an important role in Nephromyces metabolism.

As more apicomplexan genomes are sequenced it is becoming apparent that while they do share a large core subset of proteins, the differential losses and 
expansions are very lineage specific. This is likely due to adaptations required for specific host biology. Each lineage displays a characteristic patchwork of different gene losses and expansions. Many of these lineages contain orthologs with the Chromerids that are not found in other apicomplexan lineages. Nephromyces and Cardiosporidium have retained both purine biosynthesis and degradation, which has been lost in all other apicomplexan lineages. There may be something particular to ascidian biology that necessitates retaining and expressing these purine metabolism pathways. Nephromyces and Cardiosporidium share the vast majority of their genes with each other, as well as encoding the majority of the commonly shared apicomplexan genes. With so many metabolic similarities between Cardiosporidium and Nephromyces, we were unable to detect any clear differences related to Nephromyces' proposed mutualistic relationship. While there are no obvious differences between Nephromyces and Cardiosporidium, we are severely limited by a lack of lineage specific proteomic work. In other apicomplexans the gene families that modulate host immunity are highly lineage specific. In Nephromyces/Cardiosporidium, the mechanisms of host manipulation are entirely unknown. Without a greater understanding of how both Cardiosporidium and Nephromyces interact with their host at a proteomic level, we are unable to conclusively say that there is a difference between these two organisms. 


\section{Methods}

Molgula manhattensis collection

Molgula manhattensis tunicates were collected from a dock in Greenwich

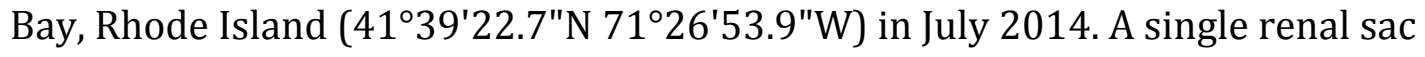
was separated from one tunicate, and all extraneous tissue removed. The intact renal sac was placed in liquid nitrogen for $5 \mathrm{~min}$ and then stored at $-80^{\circ} \mathrm{C}$.

Cardiosporidium cionae collection, isolation, and concentration

Ciona intestinalis were collected from Matunuck Marina, RI (41.3890 N, 71.5201 ${ }^{\circ} \mathrm{W}$ ), in August 2017. Tunics were removed and the body wall was opened to allow access to the heart. A sterile syringe was used to remove cardiac blood as cleanly as possible. Blood was kept at $4^{\circ} \mathrm{C}$ until Cardiosporidium infection was verified using Giemsa stain to visualize Cardiosporidium. Heavily infected samples were pooled together and centrifuged at $500 \mathrm{~g}$ for 5 minutes. The resulting supernatant was removed and the samples were frozen in liquid nitrogen and stored at $-80^{\circ} \mathrm{C}$. Samples with high rates of infection were enriched for Cardiosporidium using sucrose gradients [30, 31]. Gradients of 20, 25, 30, 35, $40 \%$ sucrose solutions in phosphate buffer were layered together. Approximately $5 \mathrm{ml}$ of tunicate blood was added to the column and centrifuged at $1750 \mathrm{~g}$ for 30 mins at $4^{\circ} \mathrm{C}$. The $25 \%$ and $30 \%$ layers were collected (based on visual screens showing high Cardiosporidium cell density and low tunicate cell density), washed with PBS twice, pelleted and then frozen in liquid nitrogen and stored at $-80^{\circ} \mathrm{C}$. 


\section{$\underline{\text { RNA Extraction }}$}

RNA extraction buffer (Zymo Research LLC. Irvine, CA) was added to samples and ground with a pestle. Following grinding, the Zymo Quick-RNA kit (Zymo Research LLC. Irvine, CA) was used and the manufacturer's protocol was followed. RNA was converted to cDNA and sequenced at the School of Medicine Genome Resource Center, University of Maryland. One paired-end RNA library was run on one lane of the Illumina HiSeq platform. Resulting in 40,606,230 from the M. manhattensis renal sac. For Cardiosporidium, three samples of $C$. intestinalis blood were used: one with unseparated blood, one enriched with cells collected at the $25 \%$ sucrose gradient, and one enriched with cells from the $30 \%$ sucrose gradient were multiplexed on one lane of the Illumina HiSeq platform, resulting in 92,250,706, 109,023,104, and 110,243,954 reads respectively. Transcriptome data was assembled and proteins were predicted with Trinity/Trinotate pipeline version 2.4.0 run on the server at Brown University Center for Computation and Visualization [32]. Reads assembled into 145674 and 109,446 contigs from M. manhattensis and C. intestinalis respectively. Protein sequences were predicted using Transdecoder [32]. Blastp was used to identify bacterial sequences from assembled transcripts against NCBI's refseq and binned. Remaining Eukaryotic sequences were separated with blastp against a custom database of alveolate and ascidian transcriptomes. Trimmed reads were mapped back to each of the six bins (Nephromyces, M. manhattensis, Nephromyces 
bacteria, Cardiosporidium, C. intestinalis, and Cardiosporidium bacteria) and then reassembled independently in Trinity. The Nephromyces transcriptome was composed of multiple Nephromyces species, and CD-hit was used to cluster transcripts based on 50 percent identity. Transcriptome completeness was assessed with Busco v3 against the Eukaryotic and bacterial reference data sets [33]. Transcripts were annotated using Interproscan [34].

\section{DNA Extraction}

The renal sacs from 8 lab grown M. manhattensis individuals were dissected and their renal fluid was pooled in a $1.5 \mathrm{ml}$ Eppendorf tube. Contents were centrifuged at $8000 \mathrm{~g}$ for $5 \mathrm{~min}$. to pellet Nephromyces cells, and following centrifugation the renal fluid was discarded. $500 \mu \mathrm{l}$ of CTAB buffer with $5 \mathrm{ul}$ of proteinase K and ceramic beads were added to the pelleted Nephromyces cells. The sample was placed in a bead beater for $3 \mathrm{~min}$. and then on a rotator for 1.5hrs at room temp. $500 \mu \mathrm{l}$ of chloroform was added, mixed gently and centrifuged for $5 \mathrm{~min}$. The top layer was removed and twice the sample volume of ice cold 100\% EtOH and 10\% sample volume of 3M sodium acetate were added to the sample and incubated a -20C overnight. The sample was centrifuged at $16000 \mathrm{~g}$ for $30 \mathrm{~min}$. and the liquid was removed. Ice cold 70\% EtOH was added and centrifuged at $16000 \mathrm{~g}$ for $15 \mathrm{~min}$. Liquid was removed and sample air dried for 2 min. DNA was re-eluted in 50ul of deionized water.

\section{Illumina Sequencing}

A nanodrop (2000c, Thermo Scientific) was used to assess DNA purity and 
DNA concentration, and a genomic gel was run to assess DNA fragmentation. Following quality control, an Illumina library was constructed. Library prep and sequencing were done at the URI Genomics and Sequencing Center (URI GSC). The completed library was sequenced on the Illumina MiSeq platform at the URI GSC and the HiSeq platform at the University of Baltimore sequencing center on three lanes.

\section{Pacific Biosciences Sequencing}

Using the contents of $150 \mathrm{M}$. manhattensis renal sacs (done in batches of 10 then pooled), the same DNA extraction protocol was performed as for Illumina sequencing. DNA was sequenced using three SMRT cells on the Pacific Biosciences platform at the University of Baltimore sequencing center. Illumina assembly

One MiSeq lane and three lanes of HiSeq, all from the same library, were trimmed using Trimmomatic [35] and then assembled using Spades [36] assembler on the URI server BlueWaves.

Pacific Biosciences assembly

Pacific Biosciences reads were error corrected using pbsuite/15.8.24 [37] on the Brown University server, Oscar. Reads were then assembled using Canu [38]. Contigs generated by Canu were combined with Illumina MiSeq/HiSeq short reads with Abyss v2.02 [39]. Nephromyces contigs were identified by mapping Nephromyces transcriptome reads using Bowtie2. Contigs with greater than 90x coverage as assessed with bedtools [40] were binned as Nephromyces. 
Bacterial endosymbiont genome assembly

Using the contigs from the Abyss assembly bacterial contigs were initially identified by hexemers using VizBin [41]. Transcriptomic reads that were identified as bacterial were mapped using Bowtie2 [42]. Bacterial contigs were separated based on a 90x coverage threshold with bbmap. Binned bacterial contigs were preliminarily annotated with Prokka [43]. Resulting annotations were run through KEGG GhostKoala to assign and separate by taxonomy. Taxon separated contig bins were merged and scaffolded using PBJelly from the PBsuite of tools [37]. Trimmed Illumina MiSeq and HiSeq reads were remapped to resulting contigs to insure accurate assembly using Bowtie2. Final assembled bacterial genomes were re-annotated with Prokka with a genus specific database. Bacterial phylogeny

16s rRNA sequences from Nephromyces bacterial endosymbiont genomes, predicted by rRNAammer, and 16S rRNA sequences from Cardiosporidium transcriptome were used in the phylogenetic analysis. All 16s rRNA rickettsiales, sphingobacteriaceae, and alcaligenaceae sequences with a minimum length of $1300 \mathrm{bp}$ available on NCBI's refseq were downloaded separately. Sequences were aligned with MAFFT [44] with G-INS-I and trimmed to length in Geneious 6. Maximum likelihood trees of the alignments were generated with RAxML v 8.2.0 using the GTRCAT model run for 10000 generations with 100 generation burn in [45].

Orthology 
The following apicomplexan and Chromerid transcriptomes were downloaded from EuPathDB: C. parvum Iowa, G. niphandrodes, B. bovis T2Bo, T. parva Muguga, P. falciparum 3D7, C. cayetanensis, E. brunetti Houghton, E. falciformis Bayer Haberkorn, E. tenella Houghton, H. hammondi HH34, N. caninum LIV, S. neurona SN3, T. gondii ME49, C. velia CCMP2878, V. brassicaformis CCMP3155. These transcriptomes were combined with Cardiosporidium and Nephromyces (clustered at the 70\% identity) Orthofinder v. 2.2.6 was used to assign transcripts to orthologous groups. 
1. Reid AJ (2014) Large, rapidly evolving gene families are at the forefront of host-parasite interactions in Apicomplexa. Parasitology 1-14. https://doi.org/10.1017/S0031182014001528

2. Janouskovec J, Keeling PJ (2016) Evolution: Causality and the origin of parasitism. Curr Biol 26:R174-R177. https://doi.org/10.1016/j.cub.2015.12.057

3. Woo YH, Ansari H, Otto TD, et al (2015) Chromerid genomes reveal the evolutionary path from photosynthetic algae to obligate intracellular parasites. Elife 4:1-41.

https://doi.org/10.7554/eLife.06974

4. Zarowiecki M, Berriman M (2015) What helminth genomes have taught us about parasite evolution. Parasitology 142 Suppl:S85-97. https://doi.org/10.1017/S0031182014001449

5. Jackson AP (2015) The evolution of parasite genomes and the origins of parasitism. Parasitology 142:S1-S5. https://doi.org/10.1017/S0031182014001516

6. Escalante a a, Ayala FJ (1995) Evolutionary origin of Plasmodium and other Apicomplexa based on rRNA genes. Proc Natl Acad Sci U S A 92:5793-7.

https://doi.org/10.1073/pnas.92.13.5793

7. Frank SA (1992) A kin selection model for the evolution of virulence. Proc R Soc L B 250:195-197

8. Frank S a (1996) Host-symbiont conflict over the mixing of symbiotic lineages. Proc Biol Sci 263:339-344. https://doi.org/10.1098/rspb.1996.0052

9. $\quad$ Frank S a (1996) Models of parasite virulence. Q Rev Biol 71:37-78. https://doi.org/10.1086/419267

10. Brunner JL, Collins JP (2009) Testing assumptions of the trade-off theory of the evolution of parasite virulence. Evol Ecol Res 11:1169-1188

11. Kada S, Lion S (2015) Superinfection and the coevolution of parasite virulence and host recovery. J Evol Biol 28:2285-2299. https://doi.org/10.1111/jeb.12753

12. Ciancio A, Scippa S, Finetti-Sialer M, et al (2008) Redescription of Cardiosporidium cionae (Van Gaver and Stephan, 1907) (Apicomplexa: Piroplasmida), a plasmodial parasite of ascidian haemocytes. Eur J Protistol 44:181-196. https://doi.org/10.1016/j.ejop.2007.11.005

13. Kumagai A, Suto A, Ito H, et al (2011) Soft tunic syndrome in the edible ascidian Halocynthia roretzi is caused by a kinetoplastid protist. Dis Aquat Organ 95:153-161. https://doi.org/10.3354/dao02372

14. Saffo MB (1982) DISTRIBUTION OF THE ENDOSYMBIONT NEPHROMYCES GIARD WITHIN THE ASCIDIAN FAMILY MOLGULIDAE. Biol Bull 162:95-104

15. Saffo MB, Lowenstam $\mathrm{H}$ a (1978) Calcareous deposits in the renal sac of a molgulid tunicate. Science 200:1166-1168. https://doi.org/10.1126/science.200.4346.1166

16. Goodbody BYI (1965) Nitrogen Excretion in Ascidiacea. Enzyme 34:299-305

17. Saffo MB (1988) Nitrogen Waste or Nitrogen Source? Urate Degradation in the Renal Sac of Molgulid Tunicates. Biol Bull 175:403. https://doi.org/10.2307/1541732

18. Lambert CC, Lambert G, Crundwell G, Kantardjieff K (1998) Uric acid accumulation in the solitary ascidian Corella inflata. J Exp Zool 282:323-331. https://doi.org/10.1002/(SICI)1097010X(19981015)282:3<323::AID-JEZ5>3.0.CO;2-O

19. Rouf MA, Lomprey RF (1968) Degradation of uric acid by certain aerobic bacteria. J Bacteriol 96:617-622

20. Thong-On A, Suzuki K, Noda S, et al (2012) Isolation and Characterization of Anaerobic Bacteria for Symbiotic Recycling of Uric Acid Nitrogen in the Gut of Various Termites. Microbes Environ 27:186-192. https://doi.org/10.1264/jsme2.ME11325

21. Middelhoven WJ, De Hoog GS, Notermans S (1989) Carbon assimilation and extracellular antigens of some yeast-like fungi. Antonie Van Leeuwenhoek 55:165-175.

https://doi.org/10.1007/BF00404756

22. Seah B, Saffo MB, Cavanaugh CM (2011) A Tripartite Animal-Protist-Bacteria Symbiosis: Culture-Independent and Phylogenetic Characterization. Harvard

23. Moran NA, Dunbar HE, Wilcox JL (2005) Regulation of transcription in a reduced bacterial genome: Nutrient-provisioning genes of the obligate symbiont Buchnera aphidicola. J Bacteriol 
187:4229-4237. https://doi.org/10.1128/JB.187.12.4229-4237.2005

24. Lopez-Sanchez MJ, Neef A, Peret J, et al (2009) Evolutionary convergence and nitrogen metabolism in Blattabacterium strain Bge, primary endosymbiont of the cockroach Blattella germanica. PLoS Genet 5:. https://doi.org/10.1371/journal.pgen.1000721

25. De Souza DJ, Bézier A, Depoix D, et al (2009) Blochmannia endosymbionts improve colony growth and immune defence in the ant Camponotus fellah. BMC Microbiol 9:1-8. https://doi.org/10.1186/1471-2180-9-29

26. Urakawa H, Dubilier N, Fujiwara Y, et al (2005) Hydrothermal vent gastropods from the same family (Provannidae) harbour E- and y -proteobacterial endosymbionts. Environ Microbiol 7:750-754. https://doi.org/10.1111/j.1462-2920.2005.00753.x

27. Marin B, Nowack ECM, Melkonian M (2005) A plastid in the making: Evidence for a second primary endosymbiosis. Protist 156:425-432. https://doi.org/10.1016/j.protis.2005.09.001

28. Philip LA, Tullman-Ercek, George G (2006) NIH Public Access. Annu Rev Microbiol 373395. https://doi.org/10.1146/annurev.micro.60.080805.142212.The

29. Hyde JE (2007) Targeting purine and pyrimidine metabolism in human apicomplexan parasites. Curr Drug Targets 8:31-47. https://doi.org/10.2174/138945007779315524

30. Ogedengbe ME, Qvarnstrom Y, da Silva AJ, et al (2015) A linear mitochondrial genome of Cyclospora cayetanensis (Eimeriidae, Eucoccidiorida, Coccidiasina, Apicomplexa) suggests the ancestral start position within mitochondrial genomes of eimeriid coccidia. Int J Parasitol 45:361-365. https://doi.org/10.1016/j.ijpara.2015.02.006

31. Arrowood MJ, Sterling CR (2016) Isolation of Cryptosporidium Oocysts and Sporozoites Using Discontinuous Sucrose and Isopycnic Percoll Gradients Author ( s ): Michael J . Arrowood and Charles R . Sterling Published by : Allen Press on behalf of The American Society of Parasitologists St. J Parasitol 73:314-319

32. Haas BJ, Papanicolaou A, Yassour M, et al (2014) reference generation and analysis with Trinity

33. Simão FA, Waterhouse RM, Ioannidis P, et al (2015) BUSCO: Assessing genome assembly and annotation completeness with single-copy orthologs. Bioinformatics 31:3210-3212.

https://doi.org/10.1093/bioinformatics/btv351

34. Finn RD, Attwood TK, Babbitt PC, et al (2017) InterPro in 2017-beyond protein family and domain annotations. Nucleic Acids Res 45:D190-D199. https://doi.org/10.1093/nar/gkw1107

35. Bolger A, Lohse M, Usadel B (2014) Trimmomatic: A flexible trimmer for Illumina sequence data. Bioinformatics 30:2114

36. Bankevich A, Nurk S, Antipov D, et al (2012) SPAdes: A New Genome Assembly Algorithm and Its Applications to Single-Cell Sequencing. J Comput Biol 19:455-477.

https://doi.org/10.1089/cmb.2012.0021

37. English AC, Richards S, Han Y, et al (2012) Mind the Gap: Upgrading Genomes with Pacific Biosciences RS Long-Read Sequencing Technology. PLoS One 7:1-12. https://doi.org/10.1371/journal.pone.0047768

38. Koren S, Walenz B, Berlin K, et al (2014) Canu: scalable and accurate long-read assembly via adaptive k-mer weighting and repeat separation. Genome Res 1-11.

https://doi.org/10.1101/gr.215087.116.Freely

39. Jackman SD, Vandervalk BP, Mohamadi H, et al (2017) ABySS 2 . 0 : resource-efficient assembly of large genomes using a Bloom filter. Genome Res 27:768-777.

https://doi.org/10.1101/gr.214346.116.Freely

40. Quinlan AR, Hall IM (2010) BEDTools: A flexible suite of utilities for comparing genomic features. Bioinformatics 26:841-842. https://doi.org/10.1093/bioinformatics/btq033

41. Laczny CC, Sternal T, Plugaru V, et al (2015) VizBin - An application for referenceindependent visualization and human-augmented binning of metagenomic data. Microbiome 3:1-7. https://doi.org/10.1186/s40168-014-0066-1

42. Langmead B, Salzberg SL (2012) Fast gapped-read alignment with Bowtie 2. Nat Methods 9:357

43. Seemann T (2014) Prokka: Rapid prokaryotic genome annotation. Bioinformatics 30:20682069. https://doi.org/10.1093/bioinformatics/btu153 
44. Katoh K, Standley DM (2013) MAFFT multiple sequence alignment software version 7: Improvements in performance and usability. Mol Biol Evol 30:772-780.

https://doi.org/10.1093/molbev/mst010

45. Stamatakis A (2014) RAxML version 8: A tool for phylogenetic analysis and post-analysis of large phylogenies. Bioinformatics 30:1312-1313. https://doi.org/10.1093/bioinformatics/btu033 


\section{Chapter 4}

Amplicon Sequencing reveals hyper diversity and universal multi-species infection prevalence in the genus Nephromyces

by

Christopher Paight ${ }^{1}$, \& Christopher E Lane ${ }^{1 *}$

will be submitted to the Journal of Eukaryotic Microbiology

\footnotetext{
${ }^{1}$ Department of Biological Sciences, University of Rhode Island, Kingston RI, 02881, USA.
} 


\title{
CHAPTER 4
}

\begin{abstract}
Monogenus multi-species infections are common in many Apicomplexans. However, these multi-species infections are often overlooked unless specifically targeted. Using amplicon primers designed to target 18s rRNA, COI, and 16s rRNA we attempted to quantify the species diversity and incidence of multispecies infections of Nephromyces in the tunicate host Molgula manhattensis collected in a limited geographic area from the waters of Rhode Island. Our data indicate that Nephromyces is hyper diverse and multispecies infections are nearly universal.
\end{abstract}

\section{Introduction}

Nephromyces is a genus of Apicomplexa with a symbiotic relationship with their hosts, tunicates in the family molgulidae. First described in 1874 by de Lacaze-Duthiers, Nephromyces was given several "identities" until it was finally placed in Apicomplexa using molecular phylogenetics (Saffo et al. 2010). Part of the confusion over its taxonomic affinity was because Nephromyces inhabits the renal sac, a structure unique to the molgulid tunicates. While the function of the renal sac in not understood, it contains high levels of uric acid and calcium oxalate (Saffo and Lowenstam 1978). Based on the metabolic capacity of Nephromyces, it appears to use uric acid for the purpose of primary carbon and nitrogen acquisition (Chapter 2). In order to supplement a diet of uric acid, 
Nephromyces relies on bacterial endosymbionts for the biosynthesis of metabolites from pathways missing from its genome (Chapter 3). Three different types of bacterial endosymbionts have been found in the genus Nephromyces, an alphaproteobacteria in Rickettsia, a betaproteobacteria in Bordetella, and a bacteroidetes in the family sphingobacteriaceae (Chapter 3). Despite genomic data, which indicates that these different types of bacteria are not functionally equivalent, no species of Nephromyces has been shown to have more than one type of bacterial endosymbiont (Seah et al. 2011).

Based on preliminary genomic and transcriptomic sequencing of Nephromyces it became apparent that there was a surprising amount of genetic diversity in the genus. In addition to the high levels of genetic diversity, Nephromyces also had high incidences of multi-species infections within individual renal sacs. Attempts to culture single species infections and limited species (3-5 isolates) infections in the lab were met with mixed success, but even limited species populations did poorly compared to the cultures that contained species numbers that better approximated wild samples.

To quantify the biological diversity and the incidence of multispecies Nephromyces infections found in molgulid tunicates, we used an amplicon sequencing approach. Because polymorphic 18S rDNA sequences have been reported in Plasmodium (Li et al. 1997), we targeted the Cytochrome Oxidase I (CO1) mitochondrial gene, as well as the 18S. In order to account for the endosymbiotic diversity within the Nephromyces population, we also targeted the 
bacterial 16S rRNA. Genomic data indicate that members of Rickettsia, Bordetella, and sphingobacteriaceae are endosymbionts of Nephromyces isolates, but their diversity is unknown.

\section{Methods}

Fifty Molgula manhattensis tunicates were collected from a single floating dock located in Greenwich Bay, RI (41339' 11.009" N 71² 27' 5.843" W), over a period of 4 weeks in the summer of 2016. Renal sacs were dissected out of the animals and contents were collected by a micropipette and placed in $1.5 \mathrm{ml}$ Eppendorf tubes. Dissecting tools were sterilized in a 10\% bleach solution for 15 min and then rinsed between tunicates. Sample tubes were immediately frozen in liquid nitrogen for five minutes and subsequently stored at $-80^{\circ} \mathrm{C}$.

DNA was extracted using the method described in (Chapter 2). Extracted DNA was stored at $-20^{\circ} \mathrm{C}$. The $18 \mathrm{~S}$ rRNA primers and $\mathrm{C0} 1$ primers were designed to target Nephromyces based on available genomic data (Chapter 3). The universal 16S rRNA primers from (Klindworth et al. 2013) were used to amplify the bacterial endosymbionts from Nephromyces. The Illumina adaptor sequence was added to the start of each primer resulting in the following sequences $18 \mathrm{Sf}$ (TCGTCGGCAGCGTCAGATGTGTATAAGAGACAGCGGTAATTCCAGCTCC), 18Sr (GTCTCGTGGGCTCGGAGATGTGTATAAGAGACAGTGCTTTCGCAGTAGTYYGTCTTT ), CO1f

(TCGTCGGCAGCGTCAGATGTGTATAAGAGACAGYGGWGTAGGWSCWGGWTGGA), 
C01r

(GTCTCGTGGGCTCGGAGATGTGTATAAGAGACAGACTTCWGGATGWCCAAARAA) 16Sf (TCGTCGGCAGCGTCAGATGTGTATAAGAGACAGCCTACGGGNGGCWGCAG), $16 \mathrm{Sr}$

(GTCTCGTGGGCTCGGAGATGTGTATAAGAGACAGGACTACHVGGGTATCTAATCC).

For each sample, PCR was performed with all three primer sets with the following cycle $94^{\circ} \mathrm{C} 2 \min \left(94^{\circ} \mathrm{C} 30 \mathrm{sec}, 55^{\circ} \mathrm{C} 30 \mathrm{sec}, 72^{\circ} \mathrm{C} 45 \mathrm{sec}\right) \times 35,72^{\circ} \mathrm{C} 5$ min. Resulting PCR products were visually inspected on an agarose gel and quantity estimated with a nanodrop. Twenty microliters of PCR product from each of the three primer sets was pooled into a single tube corresponding to an individual tunicate. The pooled sample was cleaned using the ampure bead purification with a $0.7 \%$ solution of Agencourt AMPure beads (Beckman Coulter). The addition of well specific adaptors, library preparation, and sequencing was done at the URI genomic sequencing center on the Illumina MiSeq platform.

Sequence data were de-multiplexed prior to analysis. Bduck from the bbmap suit of tools was used to bin reads based on C01 primers (Bushnell 2014). The universal 18S rRNA and 16S rRNA primers were too conserved for reliable binning based on primers, so reads were screened against the PR2 database using the NCBI's magicblast (Boratyn et al. 2018). Sequences with an $85 \%$ ID and $35 \%$ coverage were classified as $18 \mathrm{~S}$ sequences and binned into a new file composed of $18 \mathrm{~S}$ reads. Adaptors and primer sequences were remove from the forward and reverse reads from each of the three read sets using bduck. 
Cleaned and binned read sets were individually processed in $\mathrm{R}$ using dada2 with the pool="pseudo" setting (Callahan et al. 2016). Assembled $18 \mathrm{~S}$ and 16S were assigned taxonomies with the PR2 database (Guillou et al. 2013). Cytochrome oxidase 1 (CO1) sequences were assigned taxonomy using BLASTx against NCBI's refseq_protein database. All 18S and C01 sequences that were not apicomplexan were removed from the count table, taxonomy table, and sequence files. Remaining sequences were aligned with MAFFT (Katoh and Standley 2013) to $16 \mathrm{~S}$ rRNA sequences from the three known bacterial endosymbionts found in Nephromyces. Reference sequences were trimmed to the amplicon sequence length and CD-hit was used to cluster sequences with $85 \%$ sequence identity. All bacterial sequences, which did not cluster were deemed contamination and removed from count table, taxonomy table, and sequences file.

Remaining 18S and C01 sequences were aligned with MAFFT and trimmed to the same length. Sequences were clustered at 100\%, 99\%, 98\%, 97\%, 96\%, 95\%, 94\% sequence identity levels using CD-hit (Li and Godzik 2006). Sequences from 18S, C01 clusters and 16S bins corresponding to endosymbiont type were processed individually in R. Figures were made in R using ggplot (Wickham 2016).

\section{Results}

The amplicon sequencing run resulted in $25,895,690$ reads with average reads per sample of 137,743 . After binning there were 4,930,010 C01 reads, 
$6,491,91818 \mathrm{~S}$ reads, and $14,468,22816 \mathrm{~S}$ reads. Following assembly in dada2 and decontamination there were 1,876,107 sequences corresponding to 329 amplicon sequence variants (ASVs) for 18S, 1,522,378 sequences corresponding to 188 ASVs for C01, and 62,905 sequences with 152 ASVs for $16 \mathrm{~S}$.

Of the 329 18S ASVs there is an average of 79.26 ASVs per tunicate individual with a max of 145 ASVs and a min of 34 ASVs. When clustered at the more taxonomically relevant $98 \%$ identity there are 23 clusters with an average of 5.1 per tunicate individual with a max of 11 and a min of 3 (Figure 7). The most common ASVs were seen in $59.57 \%$ of samples the least common in $2.12 \%$, when clustered at $98 \%$ these numbers rise to $86.17 \%$ and $2.12 \%$.

There is a total of 188 C01 ASVs with an average of 52.8 ASVs per tunicate a max of 101 and a min of 16 . When clustered to the $98 \%$ identity level there are a total of 26 clusters an average of 6.24 and 10/2 max/min (Figure 8). The most common AVSs were found in 53\% of tunicates sampled and the rarest ASVs were in $2 \%$. After clustering at $98 \%$ the most common clusters were in $75.5 \%$ of tunicates the rarest in $2 \%$.

From a total of 152 16s ASVs classified as Nephromyces bacterial endosymbionts 49 were recovered from Bordetella, 89 from Rickettsia, and 14 from sphingobacteriaceae. The average number of 16S ASVs per tunicate is 13.94 with a max of 49 and a min of 0 (Figure 9). Forty percent of samples contained all three types of bacterial endosymbiont and $90 \%$ contained at least two of the bacterial endosymbiont types. There were two samples, which did not contain 


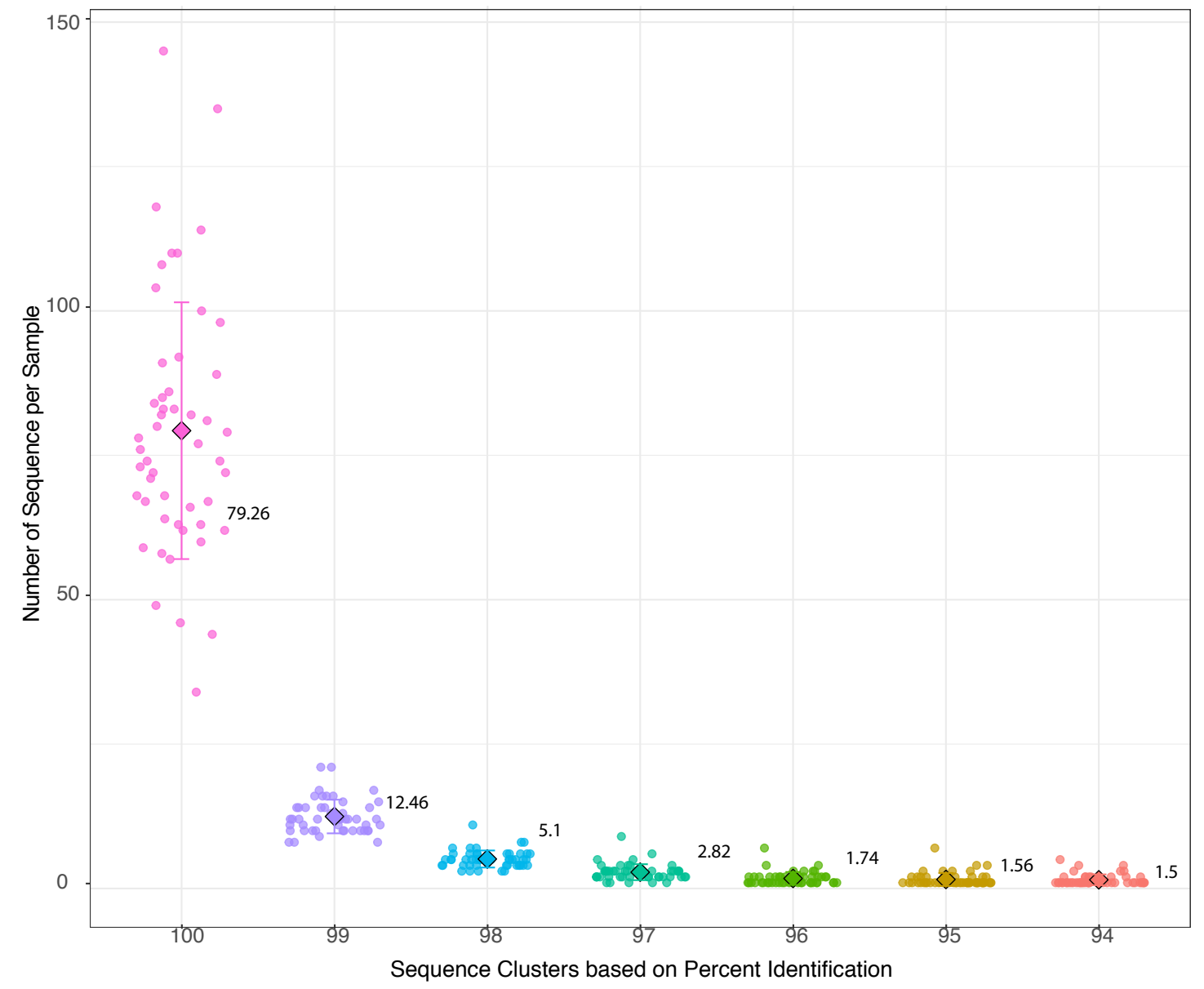

Figure 7) Grouped scatterplot of Nephromyces 18s rRNA ASV's clustered at different percent identity levels. Each dot represents the number of ASV's from an individual tunicate sample, squares indicate mean per sample (labeled to the side), and error bars indicate one standard deviation. Without clustering the average number of ASV's is 79.26 per sample and max of 145 and a min of 34 . When clustered at $98 \%$ identity the average number of sequences per sample falls to 5.1 with a max of 11 and a min of 3 . 


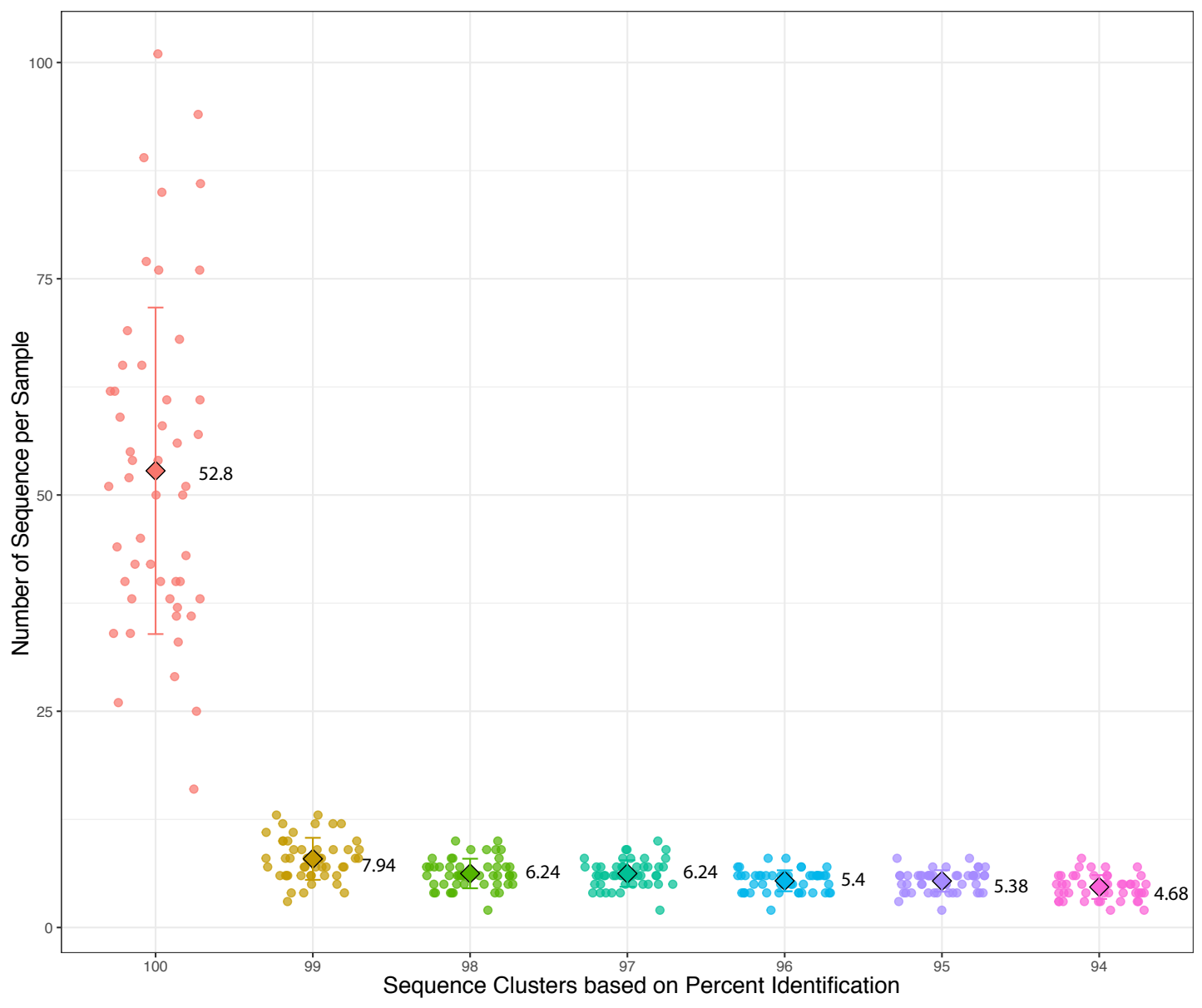

Figure 8) Grouped scatterplot of Nephromyces COI ASV's clustered at different percent identity levels. Each dot represents the number of ASV's from an individual tunicate sample, squares indicate mean per sample (labeled to the side), and error bars indicate one standard deviation. Without clustering the average number of ASV's is 52.8 per sample and max of 101 and a min of 16 . When clustered at $98 \%$ identity the average number of sequences per sample falls to 6.24 with a max of 10 and a min of 2 . 


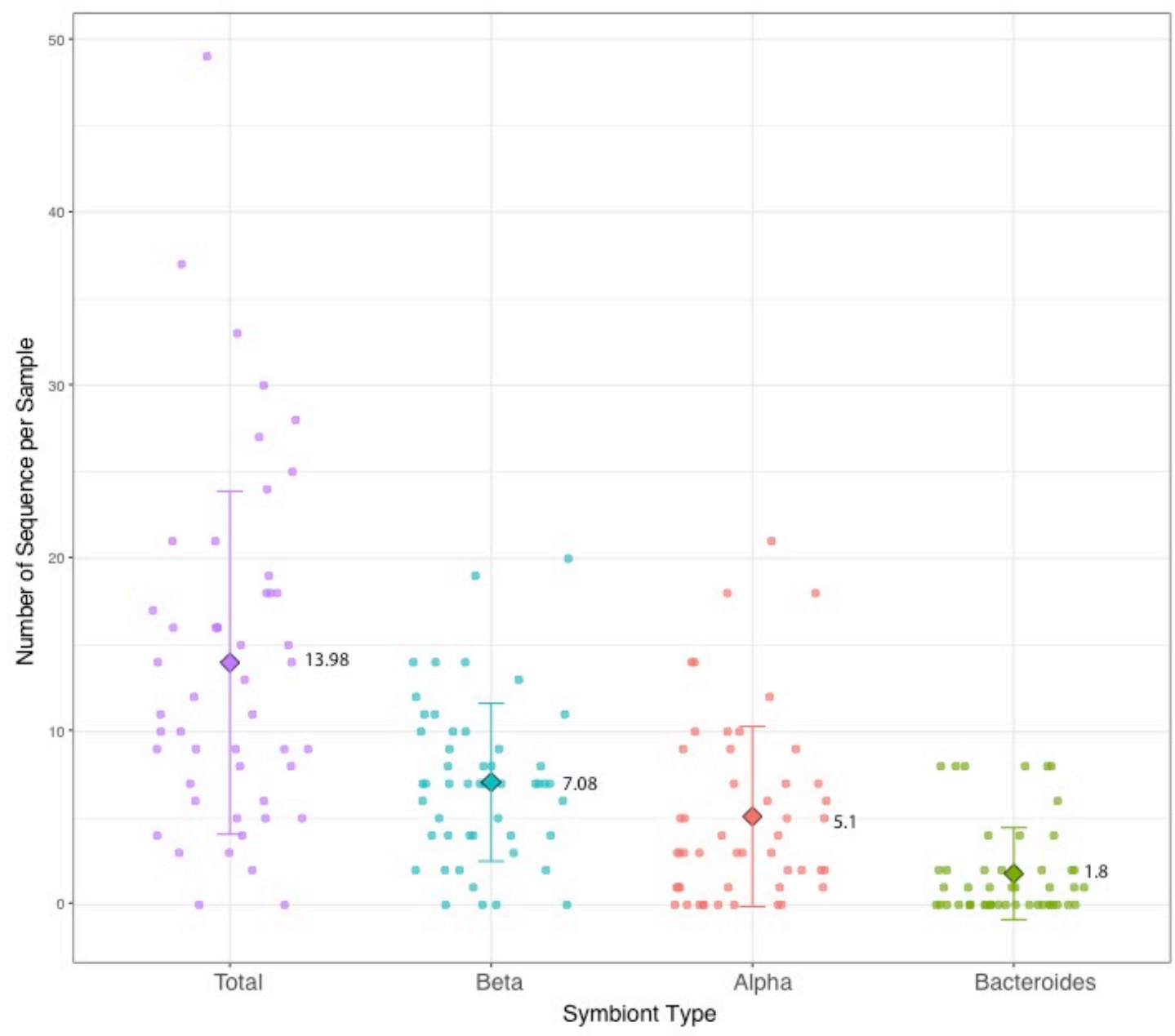

Figure 9) Grouped scatterplot of 16s rRNA ASV's from Nephromyces bacterial endosymbionts. Purple is all bacterial combined, blue is the Bordetella only, red is Rickettsia, and green in sphingobacteriaceae. Each dot represents the number of ASV's from an individual tunicate sample, squares indicate mean per sample (labeled to the side), and error bars indicate one standard deviation. An average of 13.98 bacterial ASV's per sample with 7.08 Bordetella, 5.1 Rickettsia, and 1.8 sphingobacteriaceae. 
any ASVs assigned as Nephromyces bacterial endosymbionts.

Of the 25 samples that contained two bacterial endosymbionts the Bordetella type was in 23 of the samples, Rickettsia was in 21 of the samples, and the sphingobacteriaceae type was present in 6 of the samples. Bordetella and Rickettsia were found together in 76\% (19) of renal sacs with two detected bacterial endosymbiont types, Bordetella and sphingobacteriaceae in 16\% (4), and Rickettsia and sphingobacteriaceae in 8\% (2).

\section{Discussion}

The high numbers of ASVs obtained in this study reveal that Nephromyces is extremely diverse, and in all instances, Nephromyces infections are multispecies infections. There was one renal sac that only contained a single 18S rRNA sequence, but that renal sac had multiple CO1 sequences and is likely the result of poor 18S PCR amplification of this sample. The diversity observed from amplicon sequencing is extreme, but it is supported by genomic and transcriptomic sequencing on Nephromyces (Chapter 2), as well as by cloning of full-length $18 \mathrm{~S}$ sequences. Despite the substantial diversity, the results are consistent across multiple datasets. The different number of $18 \mathrm{~S}$ and CO1 ASVs indicate that each Nephromyces species encodes multiple copies of $18 \mathrm{~S}$ with different sequences, as previously described from Plasmodium (Li et al. 1997). While CO1 is also likely found in multiple copies in a cell, based on genomic data we do not have any 
reason to suspect that $\mathrm{CO} 1$ copies differ in sequence. A similar level of diversity is observed among the $16 \mathrm{~S}$ ASVs, which is consistent with a vertically transmitted bacterial endosymbiont.

When ASVs for $18 \mathrm{~S}$ and $\mathrm{CO} 1$ are clustered at a $98 \%$ sequence identity to give an approximate species number, we estimate that there are an average of $\sim 5$ different Nephromyces species per M. manhattensis host, with some M. manhattensis containing as many as 10-11 Nephromyces species. Whereas multispecies apicomplexan infections are relatively common (Anderson et al. 2000; Lee et al. 2011; Arnott et al. 2012; Lalremruata et al. 2017), the diversity found among Nephromyces infecting a single host species is striking. The precise reasons for such high diversity are not known. However, we hypothesize that high levels of diversity may be due to the dependence of Nephromyces species on essential amino acids, co-factors, and vitamins produced by their bacterial endosymbionts.

Based on the different metabolic capabilities of the Nephromyces bacterial endosymbionts, we postulated that Nephromyces might be dependent on metabolites produced by bacterial endosymbionts in conspecifics (Chapter 2). The high proportion of tunicates containing at least two of the bacterial endosymbiont types, 90\%, supports this hypothesis. Only two samples did not contain any bacterial endosymbionts and three contained only one type of bacterial endosymbiont. This may be due to sampling error and the endosymbionts were either not amplified in the initial PCR, or one endosymbiont 
was preferentially amplified.

In samples where only two types of bacterial endosymbionts were detected the Bordetella/Rickettsia pairing were the most common ( $76 \%$ of renal sacs with two bacterial types). Based on the genomes of the endosymbionts Bordetella encodes the most complete vitamin and amino acid biosynthesis capabilities, and is predicted to be providing essential amino acids and vitamins to Nephromyces. Despite a largely complete genome assembly of the Rickettsia symbiont, its functional role is not clear. It encodes the least complete amino acid and vitamin synthesis of any of the three endosymbiont types found in Nephromyces, and all the amino acids and vitamin biosynthesis capabilities are also encoded by both the Bordetella and sphingobacteriaceae. However, given the high prevalence of Rickettsia in our samples and its presence in $82 \%$ of renal sacs with only two types of symbionts, it does appear that the Rickettsia symbionts are providing an essential function not encoded by either the Bordetella or the sphingobacteriaceae.

Based on previous (Seah et al. 2011) and our own microscopy results (Figure 10), no Nephromyces individual has been observed containing multiple types of bacterial endosymbiont. If our hypothesis is correct and Nephromyces needs metabolites from multiple symbiont types, Nephromyces species with dual endosymbionts could survive in hosts without conspecifics, as has been repeatedly observed in insects (McCutcheon and Moran 2007; Bennett and Moran 2013; Rao et al. 2015; Brown et al. 2018). One possibility is that since the 


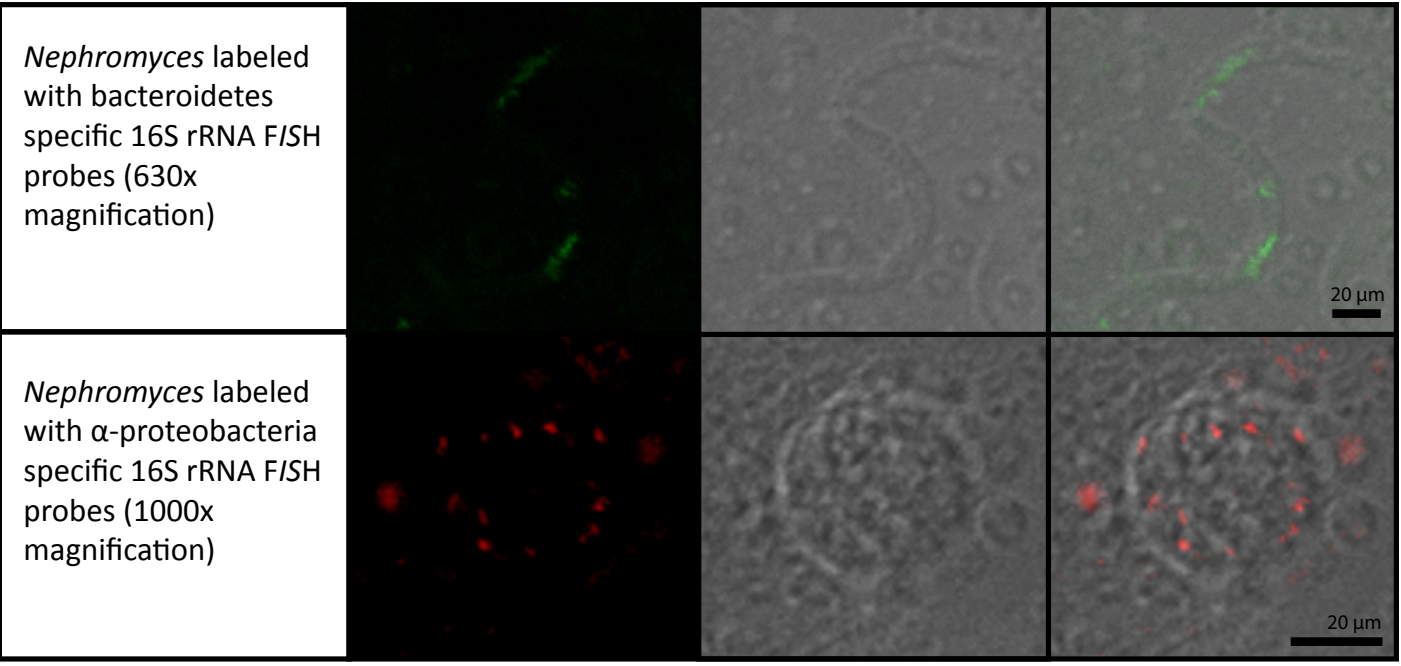

Figure 10) Photos of Nephromyces taken on a Zeiss confocal microscope labeled with two 16S rRNA FISH probes, one targeting sphingobacteriaceae (green, top, 630x) and Rickettsia (red, bottom, $1000 \mathrm{x}$ ). Images from left to right were captured under fluorescence, TPMT, and both overlaid. The top pictures are of the tachyzoite life stage and the bottom show the oocyst or merozoite stage. Bacterial endosymbionts have been observed in all of Nephromyces life stages, but no Nephromyces has been shown to contain multiple types of bacterial endosymbiont. (Courtesy of Liz Hunter) 
bacterial endosymbionts of Nephromyces are vertically transferred through the oocyst stage, there simply may not be enough space for multiple endosymbiont cells. In insects with dual endosymbionts the bacteria are typically vertically transmitted through a much larger egg cell than the oocyst stage of Nephromyces. Alternatively, it may be disadvantageous to carry multiple endosymbionts when multispecies infections are universal in molgulid renal sacs. Given the extreme Muller's ratchet known to occur in bacterial endosymbionts (Moran 1996), it may be evolutionarily cheaper to maintain one bacterial endosymbiont and rely on conspecifics with other types of bacterial endosymbiont. Such a system could not evolve unless there was a high probability of a multispecies infection of any given host.

In a system with so much co-dependence there is the potential for "cheaters" to develop and indeed we have found Nephromyces species, that do not seem to contain any bacterial endosymbiont and are presumed to parasitize the system. The absence of bacterial endosymbionts is based on single species isolates and fluorescent in situ hybridization (FISH) microscopy (Figure 6), but as the lack of signal in FISH microscopy is not definitive of absence, this has not yet been confirmed.

Given the high sequence diversity among our Nephromyces datasets, universal prevalence of multispecies infections within individual renal sacs, and that $90 \%$ of renal sac samples contain at least two types of bacterial endosymbionts, Nephromyces may not be capable of establishing single species 
infections in the wild. Combined with the metabolic capabilities of the different bacterial endosymbiont types (Chapter 2), Nephromyces likely exists as a codependent species complex or species swarm. This raises interesting questions about the evolution of co-dependence and co-evolution of Nephromyces species that require further exploration. 
Anderson T. J. C., Haubold B., Williams J. T., Estrada-Franco J. G., Richardson L., Mollinedo R., Bockarie M., Mokili J., Mharakurwa S., French N., Whitworth J., Velez I. D., Brockman A. H., Nosten F., Ferreira M. U. \& Day K. P. 2000. Microsatellite Markers Reveal a Spectrum of Population Structures in the Malaria Parasite Plasmodium falciparum. Mol Biol Evol [Internet], 17:1467-1482. Available from: http://mbe.oxfordjournals.org/cgi/content/abstract/17/10/1467

Arnott A., Barry A. E. \& Reeder J. C. 2012. Understanding the population genetics of Plasmodium vivax is essential for malaria control and elimination. Malar. J. [Internet], 11:14. Available from: http://www.malariajournal.com/content/11/1/14

Bennett G. M. \& Moran N. A. 2013. Small, smaller, smallest: The origins and evolution of ancient dual symbioses in a phloem-feeding insect. Genome Biol. Evol., 5:1675-1688.

Boratyn G. M., Thierry-Mieg J., Thierry-Mieg D., Busby B. \& Madden T. L. 2018. Magic-BLAST, an accurate DNA and RNA-seq aligner for long and short reads. bioRxiv [Internet], :390013. Available from: https://www.biorxiv.org/content/early/2018/08/14/390013 Brown A. M. V., Wasala S. K., Howe D. K., Peetz A. B., Zasada I. A. \& Denver D. R. 2018. Comparative Genomics of Wolbachia-Cardinium Dual Endosymbiosis in a Plant-Parasitic Nematode. Front. Microbiol. [Internet], 9:1-21. Available from: https://www.frontiersin.org/article/10.3389/fmicb.2018.02482/full Bushnell B. 2014. BBMap: A Fast, Accurate, Splice-Aware Aligner.

Callahan B. J., Mcmurdie P. J., Rosen M. J., Han A. W., Johnson A. J. A. \& Holmes S. P. 2016. DADA2 : High-resolution sample inference from Illumina amplicon data. Nat. Methods [Internet], :1-7. Available from: http://dx.doi.org/10.1038/nmeth.3869

Guillou L., Bachar D., Audic S., Bass D., Berney C., Bittner L., Boutte C., Burgaud G., De Vargas C., Decelle J., Del Campo J., Dolan J. R., Dunthorn M., Edvardsen B., Holzmann M., Kooistra W. H. C. F., Lara E., Le Bescot N., Logares R., Mahé F., Massana R., Montresor M., Morard R., Not F., Pawlowski J., Probert I., Sauvadet A. L., Siano R., Stoeck T., Vaulot D., Zimmermann P. \& Christen R. 2013. The Protist Ribosomal Reference database (PR2): A catalog of unicellular eukaryote Small Sub-Unit rRNA sequences with curated taxonomy. Nucleic Acids Res., 41:597-604. Katoh K. \& Standley D. M. 2013. MAFFT multiple sequence alignment software version 7: Improvements in performance and usability. Mol. Biol. Evol., 30:772780.

Klindworth A., Pruesse E., Schweer T., Peplies J., Quast C., Horn M. \& Glöckner F. 0. 2013. Evaluation of general $16 \mathrm{~S}$ ribosomal RNA gene PCR primers for classical and next-generation sequencing-based diversity studies. Nucleic Acids Res., 41:111.

Lalremruata A., Jeyaraj S., Engleitner T., Joanny F., Lang A., Bélard S., MomboNgoma G., Ramharter M., Kremsner P. G., Mordmüller B. \& Held J. 2017. Species and genotype diversity of Plasmodium in malaria patients from Gabon analysed 
by next generation sequencing. Malar. J., 16:1-11.

Lee K. S., Divis P. C. S., Zakaria S. K., Matusop A., Julin R. A., Conway D. J., Cox-Singh J. \& Singh B. 2011. Plasmodium knowlesi: Reservoir hosts and tracking the emergence in humans and macaques. PLoS Pathog., 7.

Li J., Gutell R. R., Damberger S. H., Wirtz R. A., Kissinger J. C., Rogers M. J., Sattabongkot J. \& McCutchan T. F. 1997. Regulation and trafficking of three distinct $18 \mathrm{~S}$ ribosomal RNAs during development of the malaria parasite. J. Mol. Biol., 269:203-213.

Li W. \& Godzik A. 2006. Cd-hit: A fast program for clustering and comparing large sets of protein or nucleotide sequences. Bioinformatics, 22:1658-1659.

McCutcheon J. P. \& Moran N. A. 2007. Parallel genomic evolution and metabolic interdependence in an ancient symbiosis. Proc. Natl. Acad. Sci. U. S. A. [Internet], 104:19392-19397. Available from:

http://www.pubmedcentral.nih.gov/articlerender.fcgi?artid=2148300\&tool=pm centrez\&rendertype $=$ abstract

Moran N. A. 1996. Accelerated evolution and Muller's rachet in endosymbiotic bacteria. Proc. Natl. Acad. Sci. [Internet], 93:2873-2878. Available from:

http://www.pnas.org/cgi/doi/10.1073/pnas.93.7.2873

Rao Q., Rollat-Farnier P. A., Zhu D. T., Santos-Garcia D., Silva F. J., Moya A., Latorre A., Klein C. C., Vavre F., Sagot M. F., Liu S. S., Mouton L. \& Wang X. W. 2015. Genome reduction and potential metabolic complementation of the dual endosymbionts in the whitefly Bemisia tabaci. BMC Genomics [Internet], 16:1-13. Available from:

Saffo M. B. \& Lowenstam H. a. 1978. Calcareous deposits in the renal sac of a molgulid tunicate. Science, 200:1166-1168.

Saffo M., McCoy A., Rieken C. \& Slamovits C. 2010. Nephromyces, a beneficial apicomplexan symbiont in marine animals. Proc. Natl. Acad. Sci. U. S. A., 107:16190-16195.

Seah B., Saffo M. B. \& Cavanaugh C. M. 2011. A Tripartite Animal-Protist-Bacteria Symbiosis: Culture-Independent and Phylogenetic Characterization. Harvard. Wickham H. 2016. ggplot2: Elegant Graphics for Data Analysis. Springer-Verlag New York. Available from: http://ggplot2.org 


\section{CHAPTER 5}

\section{CONCLUSION}

The impetus for this research was the characterization of Nephromyces symbiotic relationship with its host (Saffo et al 2010). In a phylum composed of parasites Nephromyces alone provided greater benefits to its host than costs. This characterization was premature and no work was done to demonstrate the details of the relationship between Nephromyces and Molgula tunicates. As is the case with most symbiosis, the relationship between organisms is complex and can be dependent on external factors. Despite the extensive research into Nephromyces there is not enough support to either confirm or refute the classification of Nephromyces/Molgula relationship as mutualistic. However, the claim of mutualism was entirely based on the unusual epidemiology of Nephromyces and our work does provide insight into how Nephromyces is able to maintain nearly universal prevalence, reach extremely high cell densities, and remain avirulent.

Central to Nephromyces epidemiology is Nephromyces ability to degrade purines into glyoxylate and subsequently convert glyoxylate into malate, pyruvate, and glycine. This pathway provides a primary nitrogen, carbon and energy source from uric acid. Malate is part of the citrate cycle critical in the generation of ATP and NADH or it can be converted into oxaloacetate for gluconeogenesis. Pyruvate is extremely versatile and is easily converted into fatty acids, acetyl-CoA, or gluconeogenesis. Glycine, the most basic amino acid, can be 
processed into other amino acids and represents nitrogen capture. Tunicates have lost the ability to degrade purines past uric acid essentially making uric acid nitrogenous waste. By using a metabolic waste product, for the host, as a primary substrate, Nephromyces can presumably utilize uric acid at little to no cost to the host. This novel substrate decouples the relationship between high parasite growth and virulence, which Nephromyces to reach high cell densities and remain avirulent.

With the exception of malate synthase Cardiosporidium encodes the same purine degradation capabilities as Nephromyces and is capable of converting uric acid into pyruvate and glycine. However, due to the different hosts, Cardiosporidium has access to far smaller quantities of uric acid than Nephromyces and therefore must rely on additional sources of nutrition from the host. Molgula storage and concentration of uric acid to the renal sac has enabled Nephromyces to develop its unusual uric acid based metabolism.

It has been proposed that the mutualistic benefit to its host is the processing of indigestible uric acid. This may be the case, but it is likely an oversimplification. First, it is unclear if Molgula ever recovers anything back from the uric acid imported into the renal sac. It is possible that valuable metabolites like amino acids or vitamins are exported out of the renal sac, but this has not been demonstrated. Second, the purpose of the renal sac has not been established. Sequestration of uric acid to the renal sac may have developed over time as a way of ridding Molgula of an apicomplexan blood parasite; by providing 
a parasite with a metabolic waste product as an alternative to infecting blood cells. In this case, Molgula benefits from losing a parasite, but this relationship is hardly mutualistic, it is more of a clever host defense mechanism. Third, Nephromyces has been shown to express xanthine dehydrogenase at high levels (99 percentile of all gene expression). Hypoxanthine is the interchange between purine recycling and purine degradation. Xanthine dehydrogenase converts hypoxanthine to xanthine and xanthine to uric acid; this represents the beginning of purine degradation. The competing enzyme, hypoxanthine-guanine phosphoribosyltransferase (HGPRT), converts hypoxanthine to inosine monophosphate (IMP) and from IMP to adenine or guanine. Since the source of uric acid within the renal sac has been shown to be from the tunicate it is curious that Nephromyces would have such high expression of xanthine dehydrogenase (Saffo 1988). A possible explanation of Nephromyces high expression of xanthine dehydrogenase is as a form of host manipulation. By outcompeting host production of HGART, Nephromyces forces greater production of uric acid than may be ideal for the host. This is potentially a cost to the host, particularly in times when purines are scarce

Almost as surprising as an apicomplexan with a uric acid based metabolism was where the genes in the purine degradation pathway come from. All other sequenced apicomplexans have lost the purine degradation pathway. It was thought that the high levels of uric oxidase measured inside the renal sac originated from the Nephromyces bacterial endosymbionts. Our data conclusively 
show that the genes involved in purine degradation are encoded in Nephromyces and Cardiosporidium genome. Additionally, these genes are not the result of a gene transfer event, but are the genes that were present when Apicomplexa split with the Chromerids. This pathway had previously been attributed to the bacterial endosymbionts, but is in fact encoded by Nephromyces/Cardiosporidium. If the bacterial endosymbionts are not being utilized for purine degradation then they must be contributing in another way.

Using 16s rRNA we have determined that the $\alpha$-proteobacteria in Nephromyces and Cardiosporidium are monophyletic and therefore present when Nephromyces/Cardiosporidium lineages split. This indicates that there may be some aspect of ascidian biology that makes maintaining a bacterial endosymbiont worthwhile. Particularly as bacterial endosymbionts are not found in other apicomplexan lineages. We have yet to determine exactly what the critical function of the $\alpha$-proteobacteria is, however because it is maintained in Cardiosporidium, which is intracellular, and in Nephromyces, which is extracellular in the renal sac, the function seems to be not exclusively connected to renal sac biology.

We do not have any genomic data on the Cardiosporidium $\alpha$ proteobacteria endosymbiont at this time, making any comparisons between Nephromyces and Cardiosporidium $\alpha$-proteobacteria endosymbiont is preliminary. The genome from the $\alpha$-proteobacteria in Cardiosporidium will need to be sequenced and assembled for more robust analysis. Preliminarily based on 
RNAseq data, Cardiosporidium $\alpha$-proteobacteria appears to have more biosynthetic capabilities than Nephromyces'. This includes several essential amino acids and vitamins not present in the $\alpha$-proteobacteria of Nephromyces.

In addition to $\alpha$-proteobacteria in the genus Rickettsia, the genus Nephromyces also maintains a $\beta$-proteobacteria in the genus Bordetella and a Bacteroidetes bacterial endosymbiont in the family Sphingobacteriaceae. We have assembled the complete genome for Nephromyces Sphingobacteriaceae endosymbiont and Nephromyces Bordetella. NBe and N $\beta$ e have the biosynthesis capabilities for a number of amino acids and vitamins, which are not encoded in the Nephromyces genome. Providing Nephromyces with amino acids and vitamins eliminates the need for Nephromyces to scavenge those metabolites from the host. Presumably, this reduces Nephromyces dependence on the host and also provides a reliable source of these metabolites, which may not be available in the renal sac.

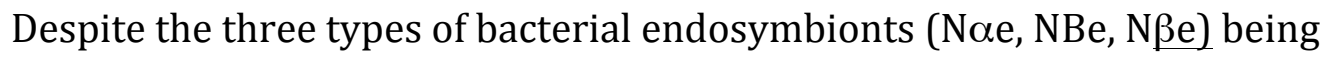
inside different Nephromyces species, we do see some similar patterns to the dual endosymbiont example in glassy winged sharpshooters. While we do not see the single pathway integration where one symbiont produces fabF and the other produces the remainder of the pathway. The lack of overlapping functions seems to indicate that despite being in different Nephromyces species, that the close proximity in the renal sac is sufficient to allow for metabolite exchange between bacterial endosymbionts in conspecific Nephromyces species. The result is 
completely unexpected and represents an unusual evolutionary quirk for the community inside the renal sac. If the renal sac community is in close enough to allow for the development of non-overlapping functions in bacterial endosymbionts in different species, it must be concluded that conspecific Nephromyces species are frequently exchanging metabolites. Based on our isolation and culturing experiments, we hypothesize that Nephromyces may be incapable of existing in isolation without conspecific Nephromyces species which contain a different type of bacterial endosymbiont than their own. We have not found a Nephromyces species containing two different types of bacterial endosymbionts, however we can't conclusively say that Nephromyces species with dual endosymbionts don't exist.

It remains unclear why a system dependent on conspecifics would develop when maintaining multiple endosymbionts would eliminate the need for competing Nephromyces species and guarantee that whatever host Nephromyces infected would be able to be colonized independent of conspecifics. Perhaps the cost of maintaining multiple endosymbionts is greater than the cost of sharing. Indeed we have found that some Nephromyces species do not maintain any endosymbiont and presumably parasitize the community, i.e. relying on the products of other Nephromyces' bacterial endosymbionts. In order for such a system to develop any given Nephromyces species must colonize a renal sac where there will be complimentary Nephromyces species. The rate at which this happens needs to be greater than the cost of maintaining two endosymbionts 
otherwise we would presumably see dual endosymbiont Nephromyces species.

A number of factors seem to contribute to the development of this codependent species complex. First, the infection prevalence of Nephromyces is nearly $100 \%$ nearly year round. Second, Nephromyces seems capable of infecting at any point in the tunicates adult life stage. Third, we have uncovered a staggering amount of Nephromyces diversity. Some individual M. manhattensis contain as many as 11 distinct Nephromyces species. Based on amplicon sequencing data there is an estimated 60 species of Nephromyces in Greenwich Bay, RI alone. The extreme amount of both species and sequence diversity in Nephromyces is not observed in Cardiosporidium and is likely connected to the unusual renal sac community dynamics. With so many species, each with a lineage of vertically inherited bacterial endosymbionts, we predict that even bacterial endosymbionts of the same type may differ widely in their metabolic capabilities. Presumably bacterial endosymbionts, even within the same taxa, could contribute different metabolites to the renal sac community. Given the tremendous amount of diversity, our sequencing of just a few of the different bacterial endosymbionts is insufficient to develop a complete picture of the intricacies of this system. It is likely that different bacterial endosymbionts within the same type may differ in metabolic capabilities.

Adding to our uncertainties we do not currently know if there are any genetic barriers preventing reproduction between different Nephromyces species. Sexual reproduction occurs inside the renal sac in the presence of multiple other 
Nephromyces species; interbreeding between species seems likely, unless there are strong genetic barriers between species. Indeed, the proximity and the interdependence of the system in general seems to indicate a great deal of interspecific breeding.

As our genomic assemblies of Nephromyces are incomplete, due in large part to the difficulties in assembling a metagenome of closely related species, we are not able to say how Nephromyces unusual epidemiology, environment, and community composition has affected its genome. Given the difficulties with Nephromyces sequencing and assembling, the genome of Cardiosporidium is a more attractive target. There are plans to sequence the genome, but currently we do not have any genomic data for Cardiosporidium. We do have good transcriptomic data for both Nephromyces and Cardiosporidium, meaning we can compare the protein coding genes of Nephromyces and Cardiosporidium to each other, as well as to other apicomplexans.

Nephromyces and Cardiosporidium have very similar metabolic capabilities based on KEGG pathway analysis. This includes purine degradation to glyoxylate and then to glycine and pyruvate, which shows that this pathway was being utilized before Nephromyces colonized the renal sac. Based on expression data, this pathway is more important for Nephromyces than Cardiosporidium. Expression of these genes in Cardiosporidium is still high $\left(80-90^{\text {th }}\right.$ percentile, the highest expression of all Cardiosporidium genes). Such high expression suggests that Cardiosporidium has access to high concentrations of uric acid inside 
tunicate blood cells, which may still represent an important source of carbon and nitrogen.

Despite the differences in epidemiology, habitat, and life histories between Nephromyces and Cardiosporidium, their metabolomes are quite similar, including the complete apicomplexan infection machinery. While Nephromyces may have a mutualistic relationship with its host, it is entering the renal sac like an apicomplexan parasite. Related to this infection machinery, both Cardiosporidium and Nephromyces have the same known dense granule, microneme, and rhoptry proteins. Proteins from these organelles have been shown in other apicomplexans to be important for invasion, immune evasion, and host manipulation. Based on Orthofinder analysis we find surprisingly few lineage-specific genes that might be involved in dealing with an ascidian immune system. We also find few genes without orthologous in either Nephromyces or Cardiosporidium compared to other apicomplexans. It is possible that these genes without orthologous are involved in the specific challenges imposed by intracellular ascidian life cycle in Cardiosporidium and the renal sac for Nephromyces. However, because these genes do not have known orthologs studied in other species, we are unable to determine function bioinformatically.

Nephromyces and Cardiosporidium have a large number of protein coding genes and metabolic capabilities. With respect to gene number and function Nephromyces/Cardiosporidium are similar to Toxoplasma. This is surprising because phylogenetically Nephromyces/Cardiosporidium belong to 
hematozoa, the lineage that contains Plasmodium, Babesia, and Theileria. The other members of hematozoa have lost peroxisomes, which we have demonstrated in Nephromyces/Cardiosporidium. Additionally, hematozoa show more metabolic reduction. Babesia, and Theileria in particular have the smallest genomes of any apicomplexan and the fewest number of protein coding genes (Kappmeyer et al. 2012; Brayton et al. 2007). The placement of Nephromyces/Cardiosporidium in hematozoa adds to the growing body of support that, these gene losses in Apicomplexa were gradual and lineage specific, with losses occurring later than predicted. These gene losses often occur in parallel in different lineages confounding assumptions based on the most parsimonious solutions. Indeed we see in nearly every apicomplexan lineage, lineage specific orthologs present only in chromerids and lost in all other apicomplexans. In the case of Nephromyces/Cardiosporidium lineage specific orthologs include purine degradation and de novo purine biosynthesis. These retained pathways appear to have particular significance to Nephromyces/Cardiosporidium, but lineage specific gene retention is consistent with the general patterns observed across the phyla Apicomplexa.

This work represents a step toward fully understanding the complexities of this unusual system, but leaves many questions unresolved. First, sequencing the genome of both Cardiosporidium and Cardiosporidiums bacterial endosymbiont would allow for more robust comparison to Nephromyces Rickettsia endosymbiont. This would provide a better understanding of the 
evolutionary history of both Nephromyces and its endosymbionts. Secondly, the biochemical pathways were based on bioinformatics with minimal confirmation at the protein level, the presented pathways need to be confirmed. Another step would be to show that uric acid is central to the metabolism of Nephromyces. A potential method to demonstrate this pathway is by injecting isotope labeled uric acid into the renal sac, and then using a new method for identifying the proteins from a specific organism in a metaproteomic sample (Kleiner et al. 2018). If this could be adapted to this system we could potentially confirm uric acid as the primary carbon and nitrogen source for Nephromyces, and determine the metabolites exchanged with the bacterial endosymbiont. This could also show if any of the carbon or nitrogen from uric acid makes its way across the renal wall back to the tunicate. If useful metabolites are exported or leaked out of the renal sac this would be the best support yet that the relationship between Nephromyces and Molgula is in fact mutualistic. 


\section{APPENDICES}

\begin{tabular}{|c|c|c|c|c|c|}
\hline Gene & PSORTII & Ppero pts1 & TargetP & topcons & predotar \\
\hline MLS & cyto: 8 , extr: 5, chlo: 1 & No & - & Yes & possibly plastid \\
\hline MLS & cyto: 11 , nucl: 2 , vacu: 1 & No & - & Yes & none \\
\hline MLS & chlo: 5.5 , chlo_mito: $5.3:$ & No & $\bar{M}$ & No & possibly mitochondrial \\
\hline MLS & chlo: 9 , mito: $\overline{4}$, plas: 1 & No & $M$ & No & possibly mitochondrial \\
\hline MLS & cyto: 8, nucl: 3, vacu: $1, \xi$ & No & _ & No & none \\
\hline MLS & cyto: 7 , nucl: 3 , chlo: $2, \mathrm{~g}$ & No & _ & No & none \\
\hline MLS & cyto: 8 , extr: 5, chlo: 1 & No & - & No & possibly plastid \\
\hline MLS & chlo: 6 , cyto: 3 , mito: $3, \mathrm{r}$ & No & $\bar{M}$ & No & possibly mitochondrial \\
\hline MLS & cyto: 6 , extr: 3 , chlo: $1, n$ & No & - & No & possibly plastid \\
\hline MLS & chlo: 5.5 , chlo_mito: $5.3:$ & No & $\bar{M}$ & No & possibly mitochondrial \\
\hline $\mathrm{MDH}$ & extr: 9 , chlo: 2, nucl: $1, \mathrm{c}^{\prime}$ & No & s & No & none \\
\hline PEX1 & chlo: 5 , nucl: 2 , cyto: $2, p$ & No & $S$ & No & none \\
\hline PEX2 & nucl: 13.5 , cyto_nucl: 7.5 & No & _ & No & none \\
\hline PEX2 & nucl: 6 , cyto: 5 , mito: $1, \epsilon$ & No & - & No & none \\
\hline PEX4 & cyto: 5 , chlo: 3 , nucl: $2, p$ & No & - & No & none \\
\hline PEX4 & cyto: 5 , chlo: 3 , nucl: $2, p$ & No & - & No & none \\
\hline PEX5 & mito: 7 , chlo: 4 , nucl: $2, c$ & No & $\bar{M}$ & No & possibly mitochondrial \\
\hline PEX5 & plas: 5 , nucl_plas: $4.5, \mathrm{cy}$ & No & _ & No & none \\
\hline PEX7 & nucl: 7 , cyto: 7 & No & - & No & none \\
\hline PEX7 & nucl: 14 & No & - & No & none \\
\hline PEX7 & nucl: 14 & No & - & No & none \\
\hline PEX7 & extr: 7 , nucl: 4 , chlo: $2, \mathrm{c}^{\prime}$ & No & - & No & none \\
\hline PEX7 & extr: 7 , nucl: 4 , chlo: $2, \mathrm{c}^{\prime}$ & No & - & No & none \\
\hline PEX10 & nucl: 12 , extr: 2 & No & $\bar{S}$ & No & possibly ER \\
\hline PEX12 & plas: 7, E.R.: 4, cyto: $1, \mathrm{~m}$ & No & _- & No & none \\
\hline PEX12 & nucl: 3 , extr: 3 , vacu: $2, E$ & No & - & No & none \\
\hline PEX12 & nucl: 3 , extr: 3 , vacu: $2, E$ & No & - & No & none \\
\hline PEX14 & nucl: 10 , cyto: 3, plas: 1 & No & - & No & none \\
\hline PEX14 & pero: 11 , cyto_nucl: $2, \mathrm{n}$ & No & - & No & none \\
\hline MPV17 & E.R.: 5 , plas: 3 , chlo: 2 , va & Perhaps yes & & No & possibly plastid \\
\hline MPV17 & plas: 8 , vacu: 4, E.R.: 2 & No & $\bar{S}$ & No & none \\
\hline MPV17 & chlo: 4 , nucl: 3 , vacu: 2 , E & No & S & No & ER \\
\hline$A B C D$ & plas: 9 , vacu: 2 , nucl: $1, \mathrm{c}$ & No & _ & No & none \\
\hline$A B C D$ & plas: 5, vacu: 4, E.R.: 2, n & No & & No & none \\
\hline$A B C D$ & plas: 9 , mito: 2 , chlo: $1, \mathrm{E}$ & No & - & No & none \\
\hline$A B C D$ & chlo: 4 , vacu: 4 , plas: $2, r$ & No & - & No & none \\
\hline$A B C D$ & chlo: 4 , vacu: 4 , plas: $2, r$ & No & - & No & none \\
\hline ABCD3 & chlo: 7 , cyto: 3 , mito: 3, & No & - & No & none \\
\hline
\end{tabular}




\begin{tabular}{|c|c|c|c|c|c|}
\hline $\mathrm{ABCD} 3$ & plas: 8, vacu: 3, E.R.: 2, n & No & - & No & possibly ER \\
\hline ACAA1 & cyto: 8 , chlo: 2, nucl: $1, \mathrm{n}$ & No & - & No & none \\
\hline ACAA1 & chlo: 4 , cyto: 3.5 , golg: 3 & No & - & No & none \\
\hline ACAA1 & chlo: 4 , cyto: 3.5 , golg: 3 & No & - & No & none \\
\hline ACAA1 & cyto: 8 , chlo: 2, nucl: $1, n$ & No & - & No & none \\
\hline ACAA1 & chlo: 11.5, chlo_mito: 7 , & No & $\mathrm{M}$ & No & mitochondrial \\
\hline ACAA1 & chlo: 9, mito: 3, nucl: 2 & No & _- & No & none \\
\hline ACAA1 & chlo: 11.5, chlo_mito: 7 , & No & $\mathrm{M}$ & No & mitochondrial \\
\hline ACAA1 & chlo: 5 , nucl: 4 , cyto: $2, \mathrm{n}$ & No & - & No & none \\
\hline ACAA1 & cyto: 6 , chlo: 4 , nucl: $2, \mathrm{p}$ & No & - & No & none \\
\hline ACAA1 & cyto: 6 , chlo: 4 , nucl: $2, \mathrm{p}$ & No & _- & No & none \\
\hline ACAA1 & chlo: 5 , nucl: 4 , cyto: $2, n$ & No & _ & Yes & none \\
\hline ACAA1 & chlo: 5 , nucl: 4 , cyto: $2, n$ & No & - & No & none \\
\hline ACAA1 & nucl: 7 , cyto: 4 , mito: 2 , c & No & - & No & none \\
\hline ACAA1 & nucl: 7 , cyto: 4 , mito: 2 , c & No & - & No & none \\
\hline ACAA1 & nucl: 7 , cyto: 4 , mito: 2 , c & No & - & No & none \\
\hline ACAA1 & nucl: 7 , cyto: 4, mito: 2 , c & No & - & No & none \\
\hline ACAA1 & nucl: 7 , cyto: 4 , mito: 2 , c & No & - & No & none \\
\hline ACAA1 & nucl: 7 , cyto: 4 , mito: 2 , c & No & - & No & none \\
\hline ACAA1 & nucl: 7 , cyto: 4 , mito: 2 , c & No & - & No & none \\
\hline ACAA1 & nucl: 6 , mito: 4 , cyto: 2 , c & No & - & No & none \\
\hline ACAA1 & nucl: 6 , mito: 4 , cyto: 2 , c & No & - & No & none \\
\hline ACOX & cyto: 9 , nucl: 2 , mito: 2, & No & - & No & none \\
\hline ACOX & cyto: 7.5, cyto_nucl: 5, E. & No & _ & No & none \\
\hline ACOX & cyto: 7.5, cyto_nucl: 5, E. & No & - & No & none \\
\hline ACOX & cyto: 9 , nucl: 2 , mito: 2, & No & - & No & none \\
\hline ACOX & pero: 11 , cyto: 2 , golg: 1 & No & - & No & none \\
\hline ACOX & pero: 11 , cyto: 2 , golg: 1 & Yes & - & No & none \\
\hline ACOX & pero: 11 , cyto: 2 , golg: 1 & Yes & - & No & none \\
\hline ACOX & pero: 11 , cyto: 2 , golg: 1 & Yes & - & No & none \\
\hline ACOX & pero: 11 , cyto: 2 , golg: 1 & Yes & - & No & none \\
\hline ACOX & pero: 11 , cyto: 2 , golg: 1 & Yes & - & No & none \\
\hline ACOX & pero: 11 , cyto: 2 , golg: 1 & Yes & - & No & none \\
\hline AGXT & chlo: 11 , mito: 3 & No & $\mathrm{M}$ & No & possibly mitochondrial \\
\hline AGXT & cyto: 7 , mito: 4 , chlo: $1, \epsilon$ & No & - & No & none \\
\hline AGXT & cyto: 8 , mito: 4 , chlo: $1, \epsilon$ & No & - & No & none \\
\hline AGXT & chlo: 9, mito: 2, pero: 2 & No & $\mathrm{M}$ & No & possibly mitochondrial \\
\hline AGXT & chlo: 9 , mito: 2, pero: 2 & No & $\mathrm{M}$ & No & possibly mitochondrial \\
\hline AK & nucl: 8 , cyto: 2.5 , cyto_E. & No & - & No & none \\
\hline AK & nucl: 8 , cyto: 2.5 , cyto_E. & No & - & No & none \\
\hline AK & cyto: 7.5, cyto_E.R.: 5, cr & No & - & No & none \\
\hline AK & cyto: 5 , nucl: 4 , chlo: $2, n$ & No & - & No & none \\
\hline ANT & nucl: 5 , chlo: 4 , cyto: 3 , e & No & - & No & none \\
\hline
\end{tabular}




\begin{tabular}{|c|c|c|c|c|c|}
\hline ANT & nucl: 6 , cyto: 4 , chlo: 2 , e & No & - & No & none \\
\hline ANT & nucl: 5 , cyto: 3.5 , cyto_E. & No & - & No & none \\
\hline ANT & nucl: 6 , cyto: 4 , chlo: $2, \mathrm{e}$ & No & _ & No & none \\
\hline ANT & nucl: 7 , cyto: 3 , chlo: $1, n$ & No & _ & No & none \\
\hline CAT & pero: 12 , cyto: 1 , golg: 1 & Yes & _- & No & none \\
\hline CAT & pero: 12 , cyto: 1 , golg: 1 & Yes & _ & No & none \\
\hline CAT & pero: 12 , cyto: 1 , golg: 1 & Yes & - & No & none \\
\hline CAT & nucl: 7 , pero: 2 , chlo: $1, r$ & No & - & No & none \\
\hline CAT & nucl: 7 , cyto: 2 , pero: 2 , c & No & _ & No & none \\
\hline CAT & nucl: 7 , cyto: 2 , pero: 2 , c & No & - & No & none \\
\hline CAT & nucl: 7 , cyto: 2 , pero: $2, \mathrm{c}$ & No & - & No & none \\
\hline $\mathrm{CHY}$ & chlo: 8 , nucl: 2 , extr: $2, p$ & No & $S$ & No & possibly ER \\
\hline $\mathrm{CHY}$ & chlo: 8 , nucl: 2 , extr: $2, p$ & No & $S$ & No & possibly ER \\
\hline $\mathrm{CHY}$ & chlo: 10 , mito: 3 , nucl: 1 & No & M & No & none \\
\hline $\mathrm{CHY}$ & chlo: 10, mito: 3 , nucl: 1 & No & M & No & none \\
\hline $\mathrm{CHY}$ & nucl: 6 , cyto: 3 , chlo: $1, \mathrm{n}$ & No & _ & No & none \\
\hline CPK & cyto: 12 , nucl: 1 , pero: 1 & No & - & No & none \\
\hline DECR2 & cyto: 5 , chlo: 4 , extr: $2, n$ & No & - & No & none \\
\hline DECR2 & chlo: 6.5 , chlo_mito: 4.5 , & Yes & $S$ & No & ER \\
\hline DECR2 & pero: 7 , cyto: 3.5 , cyto_n & Yes & M & No & none \\
\hline DECR2 & pero: 10 , cyto: 3 , golg: 1 & Yes & _ & No & none \\
\hline DECR2 & pero: 7 , cyto: 3.5 , cyto_n & Yes & M & No & none \\
\hline DECR2 & pero: 7 , cyto: 3.5 , cyto_n & Yes & M & No & none \\
\hline DECR2 & pero: 7 , chlo: 3, cyto: $3, \varepsilon$ & Yes & - & No & none \\
\hline DECR2 & pero: 7 , chlo: 3, cyto: $3, \xi$ & Yes & _ & No & none \\
\hline DECR2 & pero: 7 , chlo: 3, cyto: $3, \varepsilon$ & Yes & - & No & none \\
\hline DECR2 & pero: 7 , cyto: 3.5 , cyto_n & Yes & M & No & none \\
\hline DECR2 & pero: 7 , chlo: 3, cyto: $3, \xi$ & Yes & - & No & none \\
\hline DECR2 & cyto: 5 , chlo: 4 , extr: $2, n$ & No & _- & No & none \\
\hline DECR2 & chlo: 13 , plas: 1 & Perhaps yes & $\mathrm{S}$ & No & possibly plastid \\
\hline DECR2 & chlo: 11 , extr: 2 , cyto: 1 & Perhaps yes & $S$ & No & none \\
\hline DECR2 & pero: 7 , cyto: 3.5 , cyto_n & Yes & M & No & none \\
\hline DECR2 & pero: 7 , cyto: 3.5 , cyto_n & Yes & M & No & none \\
\hline DECR2 & pero: 7 , cyto: 3.5 , cyto_n & Yes & M & No & none \\
\hline DECR2 & pero: 7 , cyto: 3.5 , cyto_n & Yes & M & No & none \\
\hline DECR2 & pero: 7 , cyto: 3.5 , cyto_n & Yes & M & No & none \\
\hline DECR2 & pero: 7 , cyto: 3.5 , cyto_n & Yes & M & Yes & none \\
\hline DECR2 & pero: 10 , cyto: 3 , golg: 1 & Yes & - & No & none \\
\hline DECR2 & pero: 7 , cyto: 3.5 , cyto_n & Yes & M & No & none \\
\hline DECR2 & pero: 7 , cyto: 3.5 , cyto_n & Yes & M & No & none \\
\hline DECR2 & chlo: 13 , plas: 1 & Perhaps yes & $S$ & No & possibly plastic \\
\hline DECR2 & chlo: 11 , extr: 2 , cyto: 1 & Perhaps yes & $\mathrm{S}$ & No & none \\
\hline DECR2 & pero: 11 , chlo: 1 , nucl: 1 , & Perhaps yes & - & No & none \\
\hline
\end{tabular}




\begin{tabular}{|c|c|c|c|c|c|}
\hline DECR2 & \multicolumn{2}{|c|}{ pero: 11 , chlo: 1 , nucl: 1 , Perhaps yes } & _- & No & none \\
\hline DHRS4 & pero: 9, cyto: 4 , nucl: 1 & Yes & - & No & none \\
\hline DHRS4 & pero: 9, cyto: 4, nucl: 1 & Yes & - & No & none \\
\hline DHRS4 & chlo: 7, E.R.: 2.5, extr: 2, & No & $\mathrm{S}$ & No & ER \\
\hline DHRS4 & cyto: 12 , mito: 1 , cysk_nı & No & - & No & none \\
\hline DHRS4 & cyto: 10 , chlo: 2 , nucl: $1 .$. & No & - & No & none \\
\hline DHRS4 & extr: 5 , chlo: 3 , vacu: $3, r$ & No & $\mathrm{S}$ & No & ER \\
\hline DHRS4 & extr: 5 , chlo: 3 , vacu: $3, r$ & No & $\mathrm{S}$ & No & ER \\
\hline DHRS4 & chlo: 6, E.R.: 2.5, extr: 2, & No & $\mathrm{S}$ & No & ER \\
\hline DHRS4 & chlo: 7, E.R.: 2.5, extr: 2, & No & $\mathrm{S}$ & No & ER \\
\hline DHRS4 & chlo: 8 , pero: 3 , cyto: 2.5 & No & _ & No & none \\
\hline DHRS4 & pero: 6 , cyto: 3.5 , cyto_n & Yes & - & No & none \\
\hline DHRS4 & pero: 11 , cyto: 3 & Yes & _- & No & none \\
\hline DHRS4 & chlo: 12 , extr: 2 & No & _- & No & none \\
\hline DHRS4 & chlo: 8 , cyto: 2.5, cyto_n & Yes & _- & No & none \\
\hline DJP1 & cyto: 8 , nucl: 4 , mito: $1, k$ & No & - & No & none \\
\hline DJP1 & cyto: 8 , nucl: 4 , mito: $1, k$ & No & _- & No & none \\
\hline DJP1 & nucl: 10 , plas: 2 , chlo: 1 & No & - & Yes & none \\
\hline DJP1 & nucl: 10 , cyto: 4 & No & _ & No & none \\
\hline DJP1 & nucl: 6 , cyto: 6 , plas: $1, p$ & No & - & No & none \\
\hline DJP1 & nucl: 12 , cyto: 1 , plas: 1 & No & - & No & none \\
\hline E1.3.3.6 & chlo: 6 , nucl: 4 , cyto: $2, n$ & No & $\bar{M}$ & No & none \\
\hline E1.3.3.6 & extr: 7 , chlo: 4 , vacu: $1, g$ & No & _- & No & possibly mitochondrial \\
\hline E1.3.3.6 & mito: 7 , chlo: 5 , nucl: $1, c$ & No & - & No & none \\
\hline E1.3.3.6 & chlo: 7 , mito: 4 , nucl: $2, k$ & No & - & No & none \\
\hline $\mathrm{ECH}$ & mito: 8 , nucl: 5 , chlo: 1 & No & - & No & none \\
\hline $\mathrm{ECH}$ & mito: 8 , nucl: 5 , chlo: 1 & No & - & No & none \\
\hline $\mathrm{ECH}$ & pero: 4, E.R.: 3, plas: 2, C' & Yes & - & No & none \\
\hline $\mathrm{ECH}$ & pero: 5 , E.R.: 3 , plas: $2, n$ & Yes & - & No & none \\
\hline $\mathrm{ECH}$ & pero: 5 , E.R.: 3 , plas: $2, n$ & Yes & - & No & none \\
\hline $\mathrm{ECH}$ & cyto: 6 , E.R.: 3 , nucl: 2 , cl & No & - & No & none \\
\hline $\mathrm{ECH}$ & pero: 5 , E.R.: 3 , plas: 2 , C & Yes & - & No & none \\
\hline $\mathrm{ECH}$ & chlo: 5 , cyto: 3.5, cyto_n & No & _- & No & none \\
\hline $\mathrm{ECH}$ & E.R.: 3 , pero: 3 , nucl: 2.5 , & Yes & - & No & none \\
\hline $\mathrm{ECH}$ & chlo: 4 , cyto: 4 , extr: $3, p$ & No & - & No & none \\
\hline $\mathrm{ECH}$ & E.R.: 3 , pero: 3 , nucl: 2.5 , & No & - & No & none \\
\hline $\mathrm{ECH}$ & chlo: 4 , cyto: 4 , plas: 2 , e & Yes & - & No & none \\
\hline $\mathrm{ECH}$ & mito: 8 , nucl: 5 , chlo: 1 & No & - & No & none \\
\hline $\mathrm{ECH} 1$ & cyto: 5, extr: 4, E.R.: 2.5, & No & - & No & none \\
\hline $\mathrm{ECH} 1$ & pero: 4 , chlo: 3 , E.R.: $3, p$ & Yes & - & No & none \\
\hline $\mathrm{ECH} 1$ & cyto: 4, extr: 3, E.R._plas & No & - & No & none \\
\hline $\mathrm{ECH} 1$ & chlo: 6 , cyto: 3.5, cyto_n & No & - & No & none \\
\hline $\mathrm{ECH} 1$ & pero: 4, chlo: 3, E.R.: 3, $p$ & Yes & - & No & none \\
\hline
\end{tabular}




\begin{tabular}{|c|c|c|c|c|c|}
\hline $\mathrm{ECH} 2$ & pero: 12 , cyto: 2 & Yes & _- & No & none \\
\hline $\mathrm{ECH} 2$ & pero: 12 , cyto: 2 & Yes & _- & No & none \\
\hline fabG & pero: 6 , cyto: 3.5 , cyto_n & Yes & $\mathrm{M}$ & No & none \\
\hline fabG & pero: 6 , cyto: 3.5 , cyto_n & Yes & $M$ & No & none \\
\hline $\mathrm{FACL}$ & nucl: 4 , mito: 4 , chlo: $3, \mathrm{c}$ & No & _- & No & none \\
\hline FACL & nucl: 4 , mito: 4 , chlo: $3, c$ & No & - & No & none \\
\hline $\mathrm{FACL}$ & nucl: 4, mito: 4 , chlo: $3, \mathrm{c}$ & No & _- & No & none \\
\hline $\mathrm{FACL}$ & nucl: 4 , mito: 4 , chlo: $3, \mathrm{c}$ & No & _- & No & none \\
\hline $\mathrm{FACL}$ & chlo: 6 , extr: 4 , vacu: 1.5 & No & - & No & none \\
\hline $\mathrm{FACL}$ & chlo: 6 , extr: 4 , vacu: 1.5 & No & _ & No & none \\
\hline FACL & nucl: 4 , mito: 4 , chlo: $3, c$ & Yes & - & No & none \\
\hline FACL & nucl: 4 , mito: 4 , chlo: $3, c$ & No & - & No & none \\
\hline FAR & chlo: 11 , mito: 2 , nucl: 1 & No & $\overline{\mathrm{S}}$ & No & none \\
\hline FBPA & cyto: 7 , pero: 3 , chlo: 2,1 & No & _- & No & possibly mitochondrial \\
\hline FBPA & cyto: 7 , pero: 3 , chlo: 2,1 & No & - & No & possibly mitochondrial \\
\hline FBPA & cyto: 7 , pero: 3 , chlo: 2,1 & No & - & No & possibly mitochondrial \\
\hline FBPA & cyto: 7 , pero: 3 , chlo: 2,1 & No & - & No & possibly mitochondrial \\
\hline FBPA & cyto: 7 , pero: 3 , chlo: 2,1 & No & - & No & possibly mitochondrial \\
\hline FBPA & plas: 4.5, nucl_plas: $4, \mathrm{ch}$ & No & - & No & none \\
\hline FR & cyto: 6 , cysk: $\overline{6}$, nucl: 2 & Perhaps yes & - & No & none \\
\hline FR & cyto: 6 , cysk: 6 , nucl: 2 & No & - & No & none \\
\hline FR & chlo: 8 , vacu: 3 , nucl: $2, \mathrm{r}$ & No & $\bar{S}$ & No & none \\
\hline FR & chlo: 8 , vacu: 3 , nucl: $2, \mathrm{r}$ & No & $\mathrm{S}$ & No & none \\
\hline FR & chlo: 4 , nucl: 3.5 , cyto_n & No & _ & No & possibly mitochondrial \\
\hline FR & chlo: 3 , extr: 3 , nucl: $2, c^{\prime}$ & No & $\mathrm{S}$ & No & none \\
\hline FR & chlo: 10 , cyto: 2 , nucl: 1 & No & $S$ & No & ER \\
\hline FR & chlo: 10 , cyto: 2 , nucl: 1 & No & $\mathrm{S}$ & No & ER \\
\hline FR & chlo: 3 , extr: 3 , nucl: $2, c^{\prime}$ & No & $\mathrm{S}$ & No & none \\
\hline FR & chlo: 7 , cyto: 2 , plas: $2, n$ & No & $\mathrm{S}$ & No & possibly ER \\
\hline FR & chlo: 7 , cyto: 2, plas: $2, n$ & No & $\mathrm{S}$ & No & possibly ER \\
\hline G6PI & plas: 8, cyto: 2, E.R.: $2, \mathrm{cl}$ & No & _ & No & none \\
\hline G6PI & plas: 8, cyto: 2, E.R.: 2, cl & No & _- & No & none \\
\hline GAPDH & cyto: 14 & No & $\bar{M}$ & No & possibly mitochondrial \\
\hline GAPDH & pero: 6 , cyto: 3.5 , cyto_n & Perhaps yes & M & No & none \\
\hline GAPDH & cyto: 5.5 , cyto_nucl: $3 . \overline{5}$, & No & M & No & none \\
\hline GAPDH & pero: 6 , cyto: 3.5 , cyto_n & Perhaps yes & $M$ & No & none \\
\hline GAPDH & cyto: 14 & No & M & No & none \\
\hline GAPDH & cyto: 13 , mito: 1 & No & $M$ & No & none \\
\hline GAPDH & cyto: 13 , mito: 1 & No & $M$ & Yes & none \\
\hline GAPDH & cyto: 14 & No & $\mathrm{M}$ & No & possibly mitochondrial \\
\hline GAPDH & cyto: 14 & No & $M$ & No & none \\
\hline GAPDH & cyto: 5.5 , cyto_nucl: 3.5 , & No & M & No & none \\
\hline $\mathrm{GCDH}$ & mito: 4 , chlo: 3 , nucl: $2, \mathrm{c}$ & No & _ & No & none \\
\hline
\end{tabular}




\begin{tabular}{|c|c|c|c|c|c|}
\hline GK & cysk: 10 , cyto: 3 , nucl: 1 & No & - & No & none \\
\hline GNPAT & \multicolumn{2}{|c|}{ pero: 4, E.R.: 3, plas: 2, c Perhaps yes } & $\mathrm{S}$ & No & ER \\
\hline GNPAT & E.R.: 3, chlo: 2 , nucl: $2, \mathrm{cl}$ & No & $\mathrm{S}$ & No & ER \\
\hline GNPAT & pero: 11 , cyto: 2 , nucl: 1 & Yes & _ & No & none \\
\hline GNPAT & cyto: 4, E.R.: 3, nucl: $2, n$ & No & $\mathrm{s}$ & Yes & ER \\
\hline GNPAT & cyto: 4, E.R.: 3, nucl: $2, \mathrm{r}$ & No & $\mathrm{S}$ & No & ER \\
\hline GNPAT & pero: 4, E.R.: 3, nucl: $2, p$ & No & - & No & none \\
\hline GNPAT & \multicolumn{2}{|c|}{ chlo: 6 , E.R.: 3 , plas: 2 , pr Perhaps yes } & $\mathrm{S}$ & No & none \\
\hline GOT & cyto: 10 , nucl: 1 , mito: 1 , & No & _ & Yes & none \\
\hline GOT & cyto: 11 , mito: 1 , cysk: 1 , & No & - & No & none \\
\hline GOT & cysk: 8 , cyto: 4 , nucl: 2 & No & - & No & none \\
\hline GOT & cyto: 8 , cysk: 3.5 , cysk_p & No & _ & No & none \\
\hline GOT & cyto: 10 , cysk: 3 , mito: 1 & No & - & No & none \\
\hline GOT & chlo_mito: 7.33333 , mitc & No & $M$ & No & possibly mitochondrial \\
\hline GPD & pero: 8 , cyto: 3.5 , cyto_n & Yes & _ & No & none \\
\hline GPD & pero: 8 , cyto: 3.5 , cyto_n & Yes & - & No & none \\
\hline GPD & cyto: 4.5 , nucl: 3 , cyto_E. & No & - & No & none \\
\hline GPD & nucl: 5 , chlo: 3 , cyto: $3, p$ & No & - & No & possibly plastid \\
\hline GPD & cyto: 6 , chlo: 4 , nucl: $2, \mathrm{~g}$ & No & - & No & possibly plastid \\
\hline GPD & cyto: 7 , chlo: 5 , vacu: 1 , c & No & - & No & none \\
\hline GSTK1 & chlo: 6 , cyto: 3.5 , cyto_n & No & - & Yes & none \\
\hline GSTK1 & pero: 4 , cyto: 3.5, cyto_n & No & - & No & none \\
\hline GSTK1 & pero: 10 , nucl: 2 , chlo: 1 , & Yes & - & No & none \\
\hline GSTK1 & chlo: 4, pero: 4 , cyto_nur $P$ & haps yes & - & No & none \\
\hline GSTK1 & pero: 4 , cyto: 3.5, cyto_n & No & - & No & none \\
\hline GSTK1 & chlo: 6 , cyto: 3.5 , cyto_n & No & - & No & none \\
\hline GSTK1 & pero: 10 , nucl: 2 , chlo: 1 , & No & - & No & none \\
\hline GTO & nucl: 4 , cyto: 4 , mito: 4 , c & No & - & No & possibly mitochondrial \\
\hline GTO & cyto: 13 , nucl: 1 & No & $\bar{M}$ & No & possibly plastid \\
\hline GTO & mito: 6 , chlo: 3, cyto: $3, \mathrm{r}$ & No & M & No & plastid \\
\hline $\mathrm{HAO}$ & cysk: 12 , chlo: 1 , cyto: 1 & No & _- & No & none \\
\hline $\mathrm{HAO}$ & cysk: 12 , chlo: 1 , cyto: 1 & No & - & No & none \\
\hline $\mathrm{HAO}$ & cysk: 12 , chlo: 1 , cyto: 1 & No & - & No & none \\
\hline $\mathrm{HAO}$ & cyto: 6 , cysk: 3 , chlo: $2, n$ & No & - & No & none \\
\hline $\mathrm{HAO}$ & cysk: 9, nucl: 4, chlo: 1 & No & _- & No & none \\
\hline $\mathrm{HAO}$ & cyto: 8 , cysk: 2.5 , cysk_p & No & - & No & none \\
\hline $\mathrm{HAO}$ & cysk: 13, cyto: 1 & No & - & Yes & none \\
\hline $\mathrm{HAO}$ & cyto: 8 , mito: 3 , chlo: $2, k$ & No & - & Yes & none \\
\hline $\mathrm{HAO}$ & cyto: 8 , mito: 3 , chlo: $2, k$ & No & - & No & none \\
\hline $\mathrm{HAO}$ & cyto: 12, nucl: 2 & No & - & No & none \\
\hline HEX1 & cyto: 9, E.R.: 3, golg: 2 & No & - & No & none \\
\hline HEX1 & cyto: 9 , nucl: 2 , cysk: $2, \mathrm{~g}$ & No & - & No & none \\
\hline HEX1 & cyto: 6 , cysk: 4 , extr: $2, c$ & No & - & No & none \\
\hline
\end{tabular}




\begin{tabular}{|c|c|c|c|c|c|}
\hline HEX1 & cyto: 6 , cysk: 4 , extr: 2 , c & No & - & No & none \\
\hline HEX1 & cyto: 10 , extr: 2 , cysk: 1 , & No & - & No & none \\
\hline HEX1 & nucl: 4 , cyto: 4 , extr: 2 , c' & No & - & No & none \\
\hline HEX1 & nucl: 4 , cyto: 4 , extr: 2 , c' & No & - & No & none \\
\hline HEX1 & cyto: 8 , E.R.: 3, golg: 2, n & No & _ & No & none \\
\hline HEX1 & cyto: 8 , golg: 3, E.R.: 2, cl & No & - & No & none \\
\hline HEX1 & cyto: 8 , E.R.: 3, golg: 2, n & No & - & No & none \\
\hline HEX1 & cyto: 8 , golg: 3, E.R.: 2, cl & Yes & - & No & none \\
\hline HEX1 & cyto: 9, E.R.: 3, golg: 2 & No & - & No & none \\
\hline $\mathrm{HK}$ & \multicolumn{2}{|c|}{ cyto: 7 , chlo: 3 , nucl: 3, g Perhaps yes } & - & No & none \\
\hline HK & cyto: 4 , chlo: 3 , nucl: 2 , e & No & - & No & none \\
\hline HK & cyto: 8, nucl: 2 , extr: 2 , c' & No & _ & No & none \\
\hline $\mathrm{HK}$ & cyto: 8 , chlo: 2, cysk_pla: & No & - & No & none \\
\hline HK & \multicolumn{2}{|c|}{ chlo: 5.5 , nucl: 5, chlo_m Perhaps yes } & - & No & none \\
\hline HK & \multicolumn{2}{|c|}{ cyto: 8 , nucl: 2 , extr: 2 , c' Perhaps yes } & - & No & none \\
\hline HK & \multicolumn{2}{|c|}{ cyto: 7 , chlo: 3, nucl: 3, g Perhaps yes } & - & No & none \\
\hline HMGCL & cyto: 9 , cysk: 3, nucl: $1, \mathrm{~g}$ & No & _ & No & none \\
\hline HMGCL & cyto: 9 , cysk: 3, nucl: $1, \mathrm{~g}$ & No & _ & No & none \\
\hline HMGCL & cyto: 5 , extr: 5 , chlo: $2, \mathrm{n}$ & No & _ & No & none \\
\hline HMGCL & mito: 7 , chlo: 6 , nucl: 1 & No & M & No & possibly mitochondrial \\
\hline HMGCL & cyto: 9 , cysk: 3, nucl: $1, \mathrm{~g}$ & No & - & No & none \\
\hline HMGCL & cyto: 10, nucl: 2, vacu: 1 & No & - & No & none \\
\hline HMGCL & cyto: 10, nucl: 2 , vacu: 1 , & No & - & No & none \\
\hline HMGCL & cyto: 10, nucl: 2 , vacu: 1 & No & - & No & none \\
\hline HMGCL & cyto: 10, nucl: 2 , vacu: 1 , & No & - & No & none \\
\hline HPR & cyto: 10, nucl: 1 , extr: 1 , & No & - & No & none \\
\hline HSD17B4 & extr: 8, mito: 2, E.R.: 1.5, & No & $\mathrm{S}$ & No & ER \\
\hline HSD17B4 & chlo: 7 , extr: 3 , cyto: $2, n$ & No & - & No & possibly plastid \\
\hline HSD17B4 & pero: 10, chlo: 2, nucl: 1. & Yes & _ & No & possibly plastid \\
\hline HSD17B4 & pero: 10 , chlo: 2, nucl: 1. & Perhaps yes & - & No & possibly plastid \\
\hline HSD17B4 & extr: 5 , chlo: 4 , cyto: $4, n$ & No & - & No & possibly plastid \\
\hline HSD17B4 & pero: 9 , nucl: 2.5 , cyto_n & Yes & _ & No & mitochondrial \\
\hline HSD17B4 & pero: 8 , nucl: 3.5 , cyto_n & No & - & No & none \\
\hline HSD17B4 & pero: 8 , nucl: 3.5, cyto_n & No & - & No & none \\
\hline HSD17B4 & pero: 10 , cyto: 2 , nucl: 1, & Perhaps yes & - & No & none \\
\hline HSD17B4 & pero: 12 , cyto: 2 & Perhaps yes & - & No & none \\
\hline HSD17B4 & pero: 12 , cyto: 2 & Perhaps yes & & No & none \\
\hline HSD17B4 & pero: 12 , cyto: 2 & No & - & No & none \\
\hline HSD17B4 & pero: 12 , cyto: 2 & Yes & - & No & none \\
\hline HSD17B4 & pero: 13 , cyto: 1 & Yes & - & No & none \\
\hline HSD17B4 & pero: 13, cyto: 1 & Yes & - & No & none \\
\hline HSD17B4 & pero: 10 , cyto: 3 , nucl: 1 & No & - & No & none \\
\hline HSD17B4 & pero: 9 , cyto: 3, nucl: 2 & Yes & - & No & none \\
\hline
\end{tabular}




\begin{tabular}{|c|c|c|c|c|c|}
\hline IDI & cyto: 7 , mito: 2 , chlo: $1, r$ & No & - & No & none \\
\hline katE & nucl: 5 , mito: 5 , cyto: $1, k$ & No & - & No & none \\
\hline katE & nucl: 5 , mito: 5 , cyto: $1, k$ & No & - & No & none \\
\hline katE & pero: 12 , cyto: 2 & Yes & - & No & none \\
\hline katE & nucl: 9 , mito: 4 , extr: 1 & No & - & No & none \\
\hline katE & nucl: 5 , mito: 5 , cyto: 1, & No & - & No & none \\
\hline katE & nucl: 11 , mito: 2 , plas: 1 & No & - & No & none \\
\hline katE & pero: 12 , cyto: 2 & Yes & - & No & none \\
\hline katE & nucl: 11 , mito: 2 , plas: 1 & No & - & No & none \\
\hline katE & nucl: 11 , mito: 3 & No & - & No & none \\
\hline katE & nucl: 5, mito: 5, cyto: 1, & No & - & No & none \\
\hline katE & nucl: 5 , mito: 5 , cyto: 1, & No & - & No & none \\
\hline katE & nucl: 5, mito: 5 , cyto: 1, & No & - & No & none \\
\hline katE & nucl: 5 , cyto: 2 , plas: $2, \mathrm{p}$ & No & - & No & none \\
\hline katE & cyto: 7 , nucl: 2 , pero: 2,1 & No & - & No & none \\
\hline LYS4 & cyto: 7 , chlo: 3 , nucl: $2, \mathrm{v}$ & No & $\mathrm{S}$ & No & none \\
\hline LYS4 & nucl: 8 , cyto: 5 , extr: 1 & No & - & No & none \\
\hline LYS4 & cyto: 8 , chlo: 3 , nucl: 2 , c & No & - & No & none \\
\hline LYS4 & cyto: 11 , nucl: 2 , cysk: 1 & No & - & No & none \\
\hline LYS4 & nucl: 7 , cyto: 4 , chlo: $2, n$ & No & - & No & mitochondrial \\
\hline LYS4 & nucl: 5 , chlo: 4 , cyto: $4, v$ & No & - & No & none \\
\hline LYS4 & cyto: 7 , chlo: 3 , nucl: $2, v$ & No & $S$ & No & none \\
\hline LYS4 & chlo: 8 , cyto: 5 , cysk: 1 & No & - & No & possibly plastid \\
\hline LYS4 & mito: 7.5 , chlo: 6 , cyto_n & No & $\mathrm{M}$ & No & mitochondrial \\
\hline LYS4 & cyto: 5 , mito: 4 , chlo: $2, \mathrm{r}$ & No & - & No & mitochondrial \\
\hline LYS4 & cyto: 5 , mito: 4 , chlo: $2, \mathrm{r}$ & No & - & No & mitochondrial \\
\hline LYS4 & cyto: 8 , chlo: 5 , mito: 1 & No & - & No & possibly mitochondrial \\
\hline LYS4 & nucl: 4 , cyto: 4 , chlo: $3, \mathrm{n}$ & No & - & No & mitochondrial \\
\hline LYS4 & chlo: 10.5 , chlo_mito: $7 . !$ & No & $\mathrm{M}$ & No & mitochondrial \\
\hline MDAR & mito: 8 , chlo: 6 & Yes & $\mathrm{M}$ & No & mitochondrial \\
\hline MDAR & cysk: 8 , nucl: 4 , chlo: 1 , c & No & - & No & none \\
\hline MDAR & mito: 8 , chlo: 6 & No & $\mathrm{M}$ & No & mitochondrial \\
\hline MDAR & cysk: 8 , nucl: 4 , chlo: 1 , c & No & - & No & none \\
\hline MYA2 & cyto: 11 , nucl: 2 , vacu: 1 & No & - & No & none \\
\hline MYA2 & cyto: 11 , nucl: 2 , vacu: 1 & No & - & No & none \\
\hline NUDT12 & chlo: 7 , extr: 3 , cyto: $2, \mathrm{n}$ & No & - & No & none \\
\hline OPDC-PRT & extr: 9, cyto: 2 , nucl: $1, \mathrm{n}$ & No & - & No & none \\
\hline OPDC-PRT & extr: 9, cyto: 2 , nucl: 1 , n & No & - & No & none \\
\hline PAOX & cyto: 8 , nucl: 3 , chlo: $1, \mathrm{p}$ & No & $\mathrm{M}$ & No & possibly mitochondrial \\
\hline PAOX & nucl: 5 , chlo: 4 , cyto: $3, \mathrm{n}$ & Yes & - & No & none \\
\hline PAOX & nucl: 9, chlo: 2, cysk: 2, c & No & - & No & none \\
\hline PDCR & pero: 10 , chlo: 2 , cyto: 1 & haps yes & - & No & none \\
\hline PDCR & pero: 11 , cyto: 2 , nucl: 1 & Yes & - & No & none \\
\hline
\end{tabular}




\begin{tabular}{|c|c|c|c|c|c|}
\hline HSD17B4 & pero: 6 , E.R.: 3, plas: 2, c & Yes & _- & No & none \\
\hline HSD17B4 & pero: 12 , nucl: 1 , cyto: 1 & No & - & No & none \\
\hline HSD17B4 & pero: 8 , nucl: 3.5 , cyto_n & No & _ & No & none \\
\hline HSD17B4 & pero: 12 , nucl: 1 , cyto: 1 & No & _ & No & none \\
\hline HSD17B4 & pero: 12 , cyto: 2 & Yes & _ & No & none \\
\hline HSD17B4 & pero: 12 , cyto: 2 & Perhaps yes & _ & No & none \\
\hline HXGPRT & cyto: 7 , nucl: 3 , cysk: $2, \mathrm{c}$ & No & _ & No & none \\
\hline HXGPRT & cyto: 7 , nucl: 3 , cysk: $2, \mathrm{c}$ & No & - & No & none \\
\hline HXGPRT & cyto: 7 , nucl: 3 , cysk: 2 , c & No & _ & No & none \\
\hline HXGPRT & cyto: 7 , nucl: 3 , cysk: 2, c & No & _ & No & none \\
\hline HXGPRT & cyto: 7 , nucl: 3 , cysk: $2, \mathrm{c}$ & No & _ & No & none \\
\hline HXGPRT & cyto: 11 , nucl: 1 , extr: 1 , & Perhaps yes & _ & No & none \\
\hline HXGPRT & cyto: 11 , nucl: 1 , extr: 1 , & No & _ & No & none \\
\hline HXGPRT & cyto: 11 , nucl: 1 , extr: 1 , & No & - & Yes & none \\
\hline HXGPRT & cyto: 11 , nucl: 1 , extr: 1 , & No & _ & No & none \\
\hline HXGPRT & cyto: 11 , nucl: 1 , extr: 1 , & Perhaps yes & - & No & none \\
\hline HXGPRT & cyto: 11 , nucl: 1 , extr: 1 , & No & - & No & none \\
\hline HXGPRT & cyto: 11 , nucl: 1 , extr: 1 , & No & - & No & none \\
\hline HXGPRT & cyto: 11 , nucl: 1 , extr: 1 , & No & _ & No & none \\
\hline HXGPRT & cyto: 11 , nucl: 1 , extr: 1 , & No & - & No & none \\
\hline HXGPRT & cyto: 11 , nucl: 1 , extr: 1 , & No & - & No & none \\
\hline HXGPRT & cyto: 11 , nucl: 1 , extr: 1 , & No & - & No & none \\
\hline HXGPRT & cyto: 11 , nucl: 1 , extr: 1 , & No & _ & No & none \\
\hline HXGPRT & cyto: 11 , nucl: 1 , extr: 1 , & No & - & No & none \\
\hline I5PDH & cysk: 11 , cyto: 3 & No & - & No & none \\
\hline I5PDH & cysk: 11 , cyto: 3 & No & - & No & none \\
\hline IDH & cysk: 11 , cyto: 3 & No & - & No & none \\
\hline IDH & chlo_mito: 6 , chlo: $5.5, \mathrm{n}$ & No & - & No & none \\
\hline IDH & chlo_mito: 6 , chlo: $5.5, \mathrm{n}$ & No & - & Yes & none \\
\hline IDH & cysk: 14 & No & - & No & none \\
\hline IDH & cysk: 14 & No & - & No & none \\
\hline IDH & pero: 13 , cyto: 1 & Yes & - & No & none \\
\hline IDH & pero: 13 , cyto: 1 & Yes & - & No & none \\
\hline IDH & cysk: 11 , cyto: 3 & No & - & No & none \\
\hline IDH1 & nucl: 6 , cyto: 3 , chlo: $2, \mathrm{c}$ & No & - & No & none \\
\hline IDH1 & extr: 11 , cysk_plas: 1.33 & No & $S$ & No & possibly ER \\
\hline IDH1 & extr: 10, cysk_plas: 1.33 & No & $S$ & No & possibly ER \\
\hline IDH1 & pero: 12 , cyto: 2 & Yes & - & No & none \\
\hline IDH1 & cysk: 9 , chlo: 2 , cyto: $2, n$ & No & & No & none \\
\hline IDH1 & cyto: 7.5, cyto_E.R.: 4.5 , & No & - & No & none \\
\hline IDH1 & cyto: 5.5 , chlo: 5 , cyto_E. & No & - & No & none \\
\hline IDI & chlo: 5 , cyto: 3 , mito: $3, \mathrm{r}$ & No & - & No & possibly mitochondrial \\
\hline IDI & chlo: 5 , mito: 5 , cyto: 2, & No & _- & No & mitochondrial \\
\hline
\end{tabular}




\begin{tabular}{|c|c|c|c|c|c|}
\hline PDCR & pero: 11 , cyto: 2 , nucl: 1 & Yes & & No & none \\
\hline PECR & extr: 4 , mito: 3 , cyto: 2.5 & No & $\bar{S}$ & No & ER \\
\hline PECR & extr: 4 , mito: 3 , cyto: 2.5 & No & $\mathrm{S}$ & No & ER \\
\hline PEPCK & chlo: 3 , mito: 2 , plas: $2, \mathrm{E}$ & No & _ & No & none \\
\hline PEPCK & chlo: 3 , mito: 2 , plas: $2, \mathrm{E}$ & No & _ & No & none \\
\hline PEPCK & chlo: 6 , extr: 3 , vacu: $3, r$ & No & - & No & none \\
\hline PEPCK & chlo: 3 , extr: 3 , cyto: 2 , E & No & - & No & none \\
\hline PEPCK & chlo: 6 , extr: 3 , vacu: $3, r$ & No & - & No & none \\
\hline PEPCK & chlo: 3 , extr: 3 , cyto: $2, \mathrm{E}$ & No & _- & No & none \\
\hline PNC1 & chlo: 10 , cyto: 2 , nucl: 1 & No & _ & No & none \\
\hline PNC1 & chlo: 6 , cyto: 3 , extr: $3, n$ & No & _ & No & none \\
\hline PNC1 & chlo: 6 , cyto: 3 , extr: $3, n$ & No & - & No & none \\
\hline PNC1 & cyto: 10 , cysk: 3 , golg: 1 & No & _- & No & none \\
\hline PRDX1 & chlo: 4 , nucl: 3 , extr: $3, c^{\prime}$ & No & - & No & none \\
\hline PRDX1 & cyto: 9 , cysk: 2 , chlo: $1, n$ & No & _- & No & none \\
\hline PRDX1 & chlo: 4 , nucl: 3 , extr: $3, c^{\prime}$ & No & - & No & none \\
\hline PRDX1 & nucl: 7 , cyto: 6 , plas: 1 & No & _ & No & none \\
\hline PRDX1 & cyto: 6 , nucl: 3 , extr: $3, \mathrm{c}$ & No & - & No & none \\
\hline PRDX1 & cyto: 6 , nucl: 3 , extr: 3 , c & No & _ & No & none \\
\hline PXMP2 & plas: 6 , chlo: 5 , mito: $2, \mathrm{k}$ & No & $\bar{M}$ & No & possibly mitochondrial \\
\hline SHBP & cyto: 7, E.R.: 3, extr: 2, cr & No & _- & No & none \\
\hline SHBP & cyto: 7, E.R.: 3, extr: 2 , cr & No & - & No & none \\
\hline SLC25A17 & plas: 9 , golg: 2 , cyto: $1, v$ & No & - & No & none \\
\hline SLC25A17 & plas: 9 , golg: 2 , cyto: $1, v$ & No & - & No & none \\
\hline SOD2 & mito: 5.5 , nucl: 4.5 , cyto. & No & $\bar{M}$ & No & mitochondrial \\
\hline SOD2 & mito: 6 , nucl: 5.5 , cyto_n & No & $M$ & No & mitochondrial \\
\hline SOD2 & cyto: 7 , chlo: 2 , nucl: $2, n$ & No & $\mathrm{S}$ & No & possibly mitochondrial \\
\hline SOD2 & cyto: 7 , chlo: 2 , nucl: $2, n$ & No & $\mathrm{S}$ & Yes & possibly mitochondrial \\
\hline SOD2 & chlo: 4 , vacu: 3 , mito: 2 , & No & $M$ & No & possibly mitochondrial \\
\hline SOD2 & mito: 12 , chlo: 2 & No & M & No & mitochondrial \\
\hline SOD2 & chlo: 4 , vacu: 3 , mito: 2 , & No & M & No & possibly mitochondrial \\
\hline SOD2 & chlo: 4 , vacu: 3 , mito: 2 , & No & $M$ & No & possibly mitochondrial \\
\hline SOD2 & chlo: 4 , vacu: 3 , mito: 2, & No & $\mathrm{M}$ & No & possibly mitochondrial \\
\hline SOD2 & mito: 6 , cyto: 4 , pero: 2 & No & _- & No & none \\
\hline TPI & chlo: 9 , nucl: 2 , extr: $2, c^{\prime}$ & No & - & No & none \\
\hline TPI & chlo: 6 , cyto: 4 , nucl: 2 , e & No & $\bar{S}$ & Yes & none \\
\hline TPI & cyto: 4 , nucl: 3.5, mito: 3 & No & _ & No & none \\
\hline TPI & cyto: 10 , chlo: 2 , mito: 2 & No & - & No & none \\
\hline TPI & cyto: 10 , chlo: 2 , mito: 2 & No & - & No & none \\
\hline TPI & cyto: 10 , chlo: 2 , mito: 2 & No & - & No & none \\
\hline TPI & cyto: 8 , nucl: 4 , chlo: 1 , e & No & - & No & none \\
\hline TPN & nucl: 6.5 , cyto_nucl: 5.5, & No & - & No & none \\
\hline TPN & chlo: 10 , mito: 2 , nucl: 1 , & No & $\bar{S}$ & No & ER \\
\hline
\end{tabular}




\begin{tabular}{|c|c|c|c|c|c|}
\hline TPN & chlo: 10 , mito: 2 , nucl: 1 , & No & $\mathrm{S}$ & No & ER \\
\hline TPN & chlo: 12 , mito: 1 , E.R._va & No & - & No & possibly plastid \\
\hline TPN & E.R.: 14 & No & _ & Yes & possibly plastid \\
\hline TPN & nucl: 8 , mito: 3 , chlo: $1, \mathrm{c}$ & No & $M$ & No & possibly mitochondrial \\
\hline TPN & nucl: 8 , mito: 3 , chlo: $1, c$ & No & M & No & possibly mitochondrial \\
\hline TR & chlo: 8 , mito: 6 & No & $M$ & No & possibly mitochondrial \\
\hline TR & cyto: 6.5 , cyto_nucl: $4, \mathrm{cl}$ & Yes & _- & No & none \\
\hline TR & cyto: 6.5 , cyto_nucl: $4, \mathrm{cl}$ & Yes & _- & No & none \\
\hline TR & cyto: 6.5 , cyto_nucl: $4, \mathrm{cl}$ & Yes & _ & No & none \\
\hline TR & cyto: 6.5 , cyto_nucl: $4, \mathrm{cl}$ & Yes & _- & No & none \\
\hline TR & cyto: 6.5 , cyto_nucl: $4, \mathrm{cl}$ & Yes & _ & No & none \\
\hline TR & chlo: 4 , cyto: 3, extr: $3, n$ & No & - & No & none \\
\hline TR & chlo: 6 , extr: 3 , cyto: $2, n$ & No & _- & No & none \\
\hline TR & chlo: 6 , extr: 3 , cyto: $2, n$ & No & - & No & none \\
\hline TR & E.R.: 5 , cyto: 3 , mito: $2, c$ & Yes & _ & No & none \\
\hline TR & chlo: 12 , mito: 2 & No & $M$ & No & possibly mitochondrial \\
\hline TR & chlo: 8 , mito: 6 & No & M & No & possibly mitochondrial \\
\hline uaZ & cyto: 11 , cysk: 2 , chlo: 1 & No & _- & No & none \\
\hline UGP & cyto: 7, nucl: 5 , vacu: $1, \xi$ & No & - & No & none \\
\hline UGP & cyto: 7, nucl: 5, vacu: $1, \xi$ & No & - & No & none \\
\hline UGP & cyto: 10 , nucl: 2 , chlo: 1 & No & - & No & none \\
\hline UGP & cyto: 6 , nucl: 4 , chlo: $1, v$ & No & - & No & none \\
\hline UGP & chlo: 7 , cyto: 3 , nucl: $2, n$ & No & - & No & none \\
\hline UGP & chlo: 5 , nucl: 4 , cyto: $3, n$ & No & - & No & none \\
\hline UGP & chlo: 7 , cyto: 3 , nucl: $2, n$ & No & _- & No & none \\
\hline UGP & chlo: 5 , nucl: 4 , cyto: $3, n$ & No & - & No & none \\
\hline UOX & cyto: 10 , nucl: 3 , pero: 1 & No & - & No & none \\
\hline UOX & cyto: 10 , nucl: 3 , pero: 1 & No & - & No & none \\
\hline VPS1 & cyto: 9 , nucl: 4 , chlo: 1 & No & - & No & none \\
\hline $\mathrm{XDH}$ & nucl: 9 , cyto: 2 , chlo: $1, p$ & No & - & No & none \\
\hline $\mathrm{XDH}$ & nucl: 9 , cyto: 2 , chlo: $1, p$ & No & - & No & none \\
\hline $\mathrm{ZADH} 2$ & chlo: 4 , nucl: 4 , cyto: 4 , e & No & - & No & none \\
\hline $\mathrm{ZADH} 2$ & cyto: 5 , nucl: 4 , cysk: $4, \mathrm{~g}$ & No & _- & No & none \\
\hline $\mathrm{ZADH} 2$ & cyto: 7 , chlo: 2 , nucl: $2, n$ & No & _- & No & none \\
\hline ZADH2 & cyto: 9 , nucl: 2 , mito: $2, \mathrm{t}$ & No & - & No & none \\
\hline $\mathrm{ZADH} 2$ & extr: 10 , chlo: 3 , mito: 1 & No & - & No & none \\
\hline $\mathrm{ZADH} 2$ & extr: 9 , chlo: 4 , mito: 1 & No & - & No & none \\
\hline $\mathrm{ZADH} 2$ & mito: 4.5 , chlo_mito: 4.5 & No & $\begin{array}{l}- \\
-\end{array}$ & No & none \\
\hline $\mathrm{ZADH} 2$ & mito: 4.5 , chlo_mito: 4.5 & No & - & No & none \\
\hline $\mathrm{ZADH} 2$ & chlo: 6 , extr: 6 , cyto: $1, v$ & Yes & _ & No & none \\
\hline ZADH2 & chlo: 10 , mito: 2 , extr: 2 & No & $\bar{M}$ & No & none \\
\hline
\end{tabular}


Appendix Table 2) Cardiosporidium peroxisomal-related genes Identified PeroxDB and KAAS. Complete transcripts were run through Wolf PSORTII, Ppero, TargetP, Topcons and Predotar to identify possible signal motifs inclding PTS1

\begin{tabular}{|c|c|c|c|c|c|}
\hline & & & & & \\
\hline Gene & PSORTII & Ppero pts1 & TargetP & topcons & predotar \\
\hline PEX4 & chlo: 6 , nucl: 5 , cyto: 2 & No & _- & No & none \\
\hline PEX5 & mito: 5 , chlo: 4 , nucl: 2 & No & _ & No & none \\
\hline PEX5 & cyto: 7 , nucl: 4 , plas: 3 & No & - & No & none \\
\hline PEX5 & mito: 5 , chlo: 3, cyto: 3 & No & _ & No & none \\
\hline PEX6 & cyto: 7 , nucl: 3 , chlo: 2 , & Perhaps yes & - & No & none \\
\hline PEX7 & chlo: 7 , nucl: 3 , mito: 3 & No & - & No & ER \\
\hline PEX7 & chlo: 7 , nucl: 3 , mito: 3 & No & - & No & none \\
\hline PEX7 & chlo: 7 , nucl: 3 , mito: 3 & No & _ & No & none \\
\hline PEX7 & chlo: 7 , nucl: 3 , mito: 3 & No & - & No & none \\
\hline PEX7 & chlo: 7 , nucl: 3 , mito: 3 & No & _ & No & none \\
\hline PEX7 & chlo: 7 , nucl: 3 , mito: 3 & No & - & No & none \\
\hline PEX7 & chlo: 7 , nucl: 3 , mito: 3 & No & - & Yes & none \\
\hline PEX7 & chlo: 7 , nucl: 3 , mito: 3 & No & _ & No & none \\
\hline PEX10 & cyto: 6 , nucl: 4 , chlo: 1 , & No & _ & No & none \\
\hline PEX10 & cyto: 6 , nucl: 4 , chlo: 1 , & No & _ & No & none \\
\hline PEX10 & cyto: 6 , nucl: 4 , chlo: 1 , & No & _ & Yes & none \\
\hline PEX10 & mito: 7 , chlo: 5 , nucl: 2 & No & - & Yes & none \\
\hline PEX14 & cyto: 6 , chlo: 3 , nucl: 2 & No & - & No & none \\
\hline PEX14 & chlo: 7 , cyto: 2 , nucl: 1 , & No & _ & No & none \\
\hline MPV17 & mito: 4, E.R.: 4, chlo: 3, & No & M & No & possibly mitochondrial \\
\hline$A B C D$ & vacu: 10 , golg: 2 , plas: : & No & _ & No & possibly plastid \\
\hline ACAA1 & nucl: 3, mito: 3, E.R.: 2. & No & _ & No & none \\
\hline ACAA1 & chlo: 13.5 , chlo_mito: $\overline{-}$ & No & $\bar{M}$ & No & none \\
\hline ACAA1 & cyto: 8 , chlo: 3 , nucl: 1 , & No & _ & Yes & none \\
\hline ACAA1 & nucl: 4.5 , cyto_nucl: $3 . !$ & No & C & No & none \\
\hline ACAA1 & chlo: 12 , nucl: 1 , plas: 1 & No & _ & No & none \\
\hline ACAA1 & chlo: 12 , nucl: 1 , plas: 1 & No & - & No & none \\
\hline ACAA1 & nucl: 4.5 , cyto_nucl: 4 , & No & C & No & none \\
\hline AcOX & chlo: 9, cyto: 2, plas: 2 & No & - & Yes & none \\
\hline Acox & pero: 4, E.R.: 3, plas: 2 , & Perhaps yes & - & No & none \\
\hline AGPS & cyto: 7 , cysk: 3 , mito: 2 & No & - & No & none \\
\hline AGPS & cysk: 8, nucl: 3 , cyto: 3 & No & _ & No & none \\
\hline AGPS & cyto: 7 , cysk: 4 , nucl: 3 & No & - & No & none \\
\hline AGPS & cyto: 7 , cysk: 3 , mito: 2 & No & - & No & none \\
\hline AGXT & cyto: 6 , chlo: 4 , mito: 3 & No & - & No & none \\
\hline ANT & chlo: 3 , nucl: 3 , mito: 3 & No & - & No & none \\
\hline ANT & chlo: 3 , nucl: 3 , mito: 3 & No & - & No & none \\
\hline CAT & pero: 13 , cyto: 1 & Yes & - & No & none \\
\hline CPK & cyto: 5 , nucl: 4 , plas: 2 & No & $\bar{S}$ & No & none \\
\hline
\end{tabular}




\begin{tabular}{|c|c|c|c|c|c|}
\hline CPK & cyto: 5 , nucl: 4 , plas: 2 , & No & $\mathrm{S}$ & No & none \\
\hline CPK & cyto: 5 , nucl: 4 , plas: 2 , & No & $S$ & No & none \\
\hline CPK & cyto: 5 , nucl: 4 , plas: 2 , & No & $S$ & No & none \\
\hline CPK & chlo: 4 , nucl: 3 , cyto: 2 & No & _ & No & ER \\
\hline DJP1 & nucl: 11 , chlo: 1 , plas: 1 & No & - & No & none \\
\hline DJP1 & nucl: 6 , cyto: 3, mito: 3 & No & _- & No & none \\
\hline DJP1 & mito: 5 , nucl: 3.5 , cyto: & No & _ & No & none \\
\hline DJP1 & cyto: 6 , nucl: 3, mito: 2 & No & - & No & none \\
\hline DJP1 & cyto: 5.5 , golg: 4 , cyto_ & No & _- & No & none \\
\hline E1.3.3.6 & chlo: 8 , mito: 3 , cyto: 2 & No & $\bar{M}$ & No & none \\
\hline $\mathrm{ECH}$ & mito: 5 , chlo: 2 , nucl: 2 & No & _- & No & none \\
\hline $\mathrm{ECH}$ & cyto: 7 , chlo: 2 , nucl: 2 & Yes & _- & No & none \\
\hline $\mathrm{ECH}$ & nucl: 6 , mito: 5 , chlo: 2 & No & - & No & none \\
\hline $\mathrm{ECH}$ & nucl: 6 , mito: 5 , chlo: 2 & No & - & No & none \\
\hline EHHADH & chlo: 12 , cyto: 1 , mito: & No & $\mathrm{M}$ & No & none \\
\hline FBPA & chlo: 5 , cyto: 4 , extr: 3 & No & _- & Yes & none \\
\hline FBPA & cyto: 10 , chlo: 2 , nucl: : & No & - & No & none \\
\hline FR & cyto: 8 , nucl: 3 , extr: 1 , & No & - & No & none \\
\hline FR & cyto: 8 , nucl: 3 , extr: 1 , & No & - & No & none \\
\hline FR & chlo: 4 , nucl: 4 , mito: 4 & No & - & No & none \\
\hline FR & chlo: 4 , nucl: 4 , mito: 4 & No & - & No & none \\
\hline FR & chlo: 4 , nucl: 4 , mito: 4 & No & - & No & none \\
\hline FR & chlo: 4 , nucl: 4 , mito: 4 & No & - & No & none \\
\hline FR & chlo: 13, cyto: 1 & No & $\mathrm{S}$ & No & none \\
\hline G6PI & cyto: 5 , chlo: 4 , nucl: 3 , & No & $M$ & No & mitochondrial \\
\hline G6PI & cyto: 5 , chlo: 4 , nucl: 4 , & No & M & No & none \\
\hline GAPDH & cyto: 13 , mito: 1 & No & $M$ & No & none \\
\hline GAPDH & cysk: 8 , cyto: 5 , chlo: 1 & No & _- & No & none \\
\hline GK & cysk: 11 , chlo: 2 , cyto: & Yes & - & No & none \\
\hline GK & cysk: 11 , chlo: 2 , cyto: & Perhaps yes & _ & No & none \\
\hline GOT & chlo: 10 , mito: 4 & No & $\bar{M}$ & No & none \\
\hline GOT & pero: 11 , cyto: 2 , nucl: & Yes & _ & No & none \\
\hline GOT & pero: 11 , cyto: 2 , nucl: & Perhaps yes & - & No & none \\
\hline GPD & plas: 8, golg: 3, E.R.: 2, & No & $\bar{S}$ & No & none \\
\hline GPD & plas: 8 , golg: 3, E.R.: 2, & No & $\mathrm{S}$ & No & ER \\
\hline GSTK1 & pero: 4 , cyto_nucl: $4, n$ & Yes & _ & No & ER \\
\hline GSTK1 & pero: 5 , cyto: 4.5 , cyto & Yes & - & No & none \\
\hline $\mathrm{HAO}$ & pero: 12 , cyto: 2 & Yes & - & No & none \\
\hline HEX1 & cyto: 11 , extr: 1 , cysk: 1 & No & - & No & none \\
\hline HEX1 & cyto: 11 , extr: 1 , cysk: 1 & No & - & No & none \\
\hline $\mathrm{HK}$ & chlo: 4, E.R.: 3, cyto: $2 . !$ & No & - & No & none \\
\hline $\mathrm{HK}$ & cyto: 5 , chlo: 3 , nucl: 2 , & No & - & No & none \\
\hline HK & cyto: 5.5, E.R.: 4, cyto_ & No & - & No & none \\
\hline
\end{tabular}




\begin{tabular}{|c|c|c|c|c|c|}
\hline HK & cyto: 5 , golg: 3 , mito: 2 & No & _ & No & none \\
\hline HK & nucl: 12 , cyto: 1 , mito: & No & _ & Yes & none \\
\hline HK & cyto: 5 , golg: 3 , mito: 2 & No & _ & No & none \\
\hline HMGCL & nucl: 6 , cyto: 5 , chlo: 2 , & No & _ & No & none \\
\hline HMGCL & nucl: 6 , cyto: 5 , chlo: 2 , & No & _- & No & none \\
\hline HPR & cyto: 13 , chlo: 1 & No & _- & No & mitochondrial \\
\hline HPR & cyto: 13 , nucl: 1 & No & _- & No & none \\
\hline HSD17B4 & chlo: 8 , cyto: 4 , nucl: 1 , & No & M & No & none \\
\hline HSD17B4 & cyto: 8 , chlo: 3 , pero: 2 & No & $\mathrm{M}$ & No & possibly mitochondrial \\
\hline HSD17B4 & pero: 10 , cyto: 2.5 , cytc & Yes & _ & No & possibly mitochondrial \\
\hline HSD17B4 & pero: 6 , cyto: 4.5 , cyto_ & Yes & M & No & none \\
\hline HSD17B4 & pero: 9 , cyto: 2.5 , cyto_ & Yes & _ & No & possibly mitochondrial \\
\hline HSD17B4 & chlo: 8 , nucl: 2 , pero: 2 & Yes & $\mathrm{M}$ & No & none \\
\hline HSD17B4 & chlo: 8 , nucl: 2 , pero: 2 & Yes & M & No & mitochondrial \\
\hline HSD17B4 & chlo: 8.5 , chlo_mito: 7 , & No & $\mathrm{M}$ & No & mitochondrial \\
\hline HSD17B4 & chlo: 8 , nucl: 2 , pero: 2 & Yes & $\mathrm{M}$ & No & mitochondrial \\
\hline HSD17B4 & chlo: 8 , nucl: 2 , pero: 2 & Yes & M & No & mitochondrial \\
\hline HSD17B4 & chlo: 8 , nucl: 2 , pero: 2 & Yes & M & No & mitochondrial \\
\hline HSD17B4 & pero: 11 , cyto: 2 , nucl: & Yes & _ & No & mitochondrial \\
\hline HSD17B4 & chlo: 8 , nucl: 2 , pero: 2 & Yes & M & No & none \\
\hline HSD17B4 & chlo: 8.5 , chlo_mito: 7 , & No & $\mathrm{M}$ & No & mitochondrial \\
\hline HSD17B4 & chlo: 8 , nucl: 2 , pero: 2 & Yes & $\mathrm{M}$ & No & mitochondrial \\
\hline HSD17B4 & chlo: 8 , nucl: 2 , pero: 2 & Yes & M & No & mitochondrial \\
\hline HSD17B4 & chlo: 8 , nucl: 2 , pero: 2 & Yes & $\mathrm{M}$ & No & mitochondrial \\
\hline HSD17B4 & chlo: 14 & No & $S$ & Yes & none \\
\hline I5PDH & nucl: 5 , cyto: 4 , chlo: 2 , & No & _ & No & none \\
\hline IDH & pero: 12 , cyto: 2 & Perhaps yes & - & No & none \\
\hline IDH1 & mito: 7 , chlo: 6 , cyto_n & No & $\mathrm{M}$ & No & possibly mitochondrial \\
\hline IDH1 & chlo: 6 , cyto_nucl: $3, \mathrm{nı}$ & No & _- & No & none \\
\hline IDI & chlo: 9 , mito: 2 , nucl: 1 & No & $M$ & No & none \\
\hline katE & pero: 13 , cyto: 1 & Yes & _ & No & none \\
\hline katE & pero: 9 , nucl: 2 , chlo: 1 & No & _- & No & none \\
\hline katE & pero: 8 , cyto: 3 , nucl: 1 & No & - & Yes & none \\
\hline LYS4 & cyto: 3 , nucl_plas: $3, \mathrm{nt}$ & No & _- & No & none \\
\hline LYS4 & chlo: 10 , cyto: 4 & No & _ & No & none \\
\hline LYS4 & cyto: 6 , cysk: 4 , chlo: 3 , & No & - & No & none \\
\hline LYS4 & mito: 7 , chlo: 4 , nucl: 2 & No & $M$ & No & none \\
\hline LYS4 & mito: 7.5 , cyto_mito: 4 & No & M & No & none \\
\hline MDAR & cyto: 4 , chlo: 3 , mito: 3 & No & $\mathrm{s}$ & Yes & ER \\
\hline MYA2 & nucl: 8 , cyto: 3 , chlo: 1 , & No & _- & No & none \\
\hline MYA2 & cyto: 7 , nucl: 5 , chlo: 1 , & No & _ & No & none \\
\hline MYA2 & cyto: 6 , nucl: 5 , chlo: 1 , & No & - & No & none \\
\hline MYA2 & cyto: 6 , nucl: 5 , chlo: 1 , & No & - & No & none \\
\hline
\end{tabular}




\begin{tabular}{|c|c|c|c|c|c|}
\hline OPDC-PRT & chlo: 8 , nucl: 3 , cyto: 2 & No & _- & No & ER \\
\hline PDCR & pero: 7 , cyto: 3.5 , cyto_ & Yes & - & No & possibly plastid \\
\hline PDCR & pero: 7 , cyto: 3.5 , cyto_ & Yes & _- & No & possibly plastid \\
\hline PDCR & pero: 7 , cyto: 3.5 , cyto_ & Yes & _- & No & possibly plastid \\
\hline PDCR & pero: 7 , cyto: 3.5 , cyto_ & Yes & _- & No & possibly plastid \\
\hline PEPCK & cyto: 3, E.R.: 3, nucl: 2 , & No & - & Yes & none \\
\hline PNC1 & cyto: 10 , nucl: 1 , extr: 1 & No & - & No & none \\
\hline PRDX1 & chlo: 9 , cyto: 2 , nucl: 1 & No & - & No & none \\
\hline PXMP2 & mito: 7 , chlo: 5 , nucl: 1 & No & M & Yes & ER \\
\hline SHBP & cyto: 10 , plas: 1 , extr: 1 & No & _- & No & none \\
\hline SOD2 & chlo: 9 , extr: 3 , nucl: 1 & No & $\bar{S}$ & No & ER \\
\hline SOD2 & cyto: 9 , chlo: 2 , nucl: 2 & No & $\mathrm{S}$ & No & ER \\
\hline SOD2 & chlo: 5, mito: 4, E.R.: 2, & No & M & No & possibly mitochondrial \\
\hline SOD2 & cyto: 8, nucl: 2, plas: 2 & No & - & No & none \\
\hline TPI & chlo: 4 , extr: 4 , cyto: 2 & No & $\bar{S}$ & No & none \\
\hline TPI & chlo: 4 , extr: 4 , cyto: 2 & No & $\mathrm{S}$ & No & ER \\
\hline TPN & cyto: 5 , nucl: 4 , chlo: 2 & No & _- & No & none \\
\hline TPN & cyto: 7 , chlo: 2 , nucl: 2 & No & - & No & none \\
\hline TR & vacu: 4 , chlo: 3 , mito: 2 & No & $\bar{S}$ & No & none \\
\hline TR & chlo: 5 , mito: 3 , vacu: 3 & No & $\mathrm{S}$ & No & ER \\
\hline TR & cyto: 6, E.R.: 5, mito: 2, & No & - & No & ER \\
\hline TR & cyto: 6 , E.R.: 5, mito: 2, & No & - & No & none \\
\hline uaZ & pero: 9 , cyto: 2.5 , cyto_ & Yes & - & No & none \\
\hline uaZ & pero: 11 , cyto: 2 , golg: & Yes & - & No & none \\
\hline uaZ & pero: 9 , cyto: 2.5 , cyto & Yes & - & No & none \\
\hline $\mathrm{ZADH} 2$ & chlo: 6 , nucl: 2.5 , cyto: & No & - & Yes & none \\
\hline $\mathrm{ZADH} 2$ & chlo: 6 , nucl: 2.5, cyto: & No & - & Yes & none \\
\hline ZADH2 & chlo: 9 , extr: 2 , nucl: 1 & No & $\bar{M}$ & Yes & possibly plastid \\
\hline $\mathrm{ZADH} 2$ & chlo: 6 , cyto: 6 , nucl: 1 & No & _ & Yes & none \\
\hline
\end{tabular}




\section{Biological Process GOSlim}

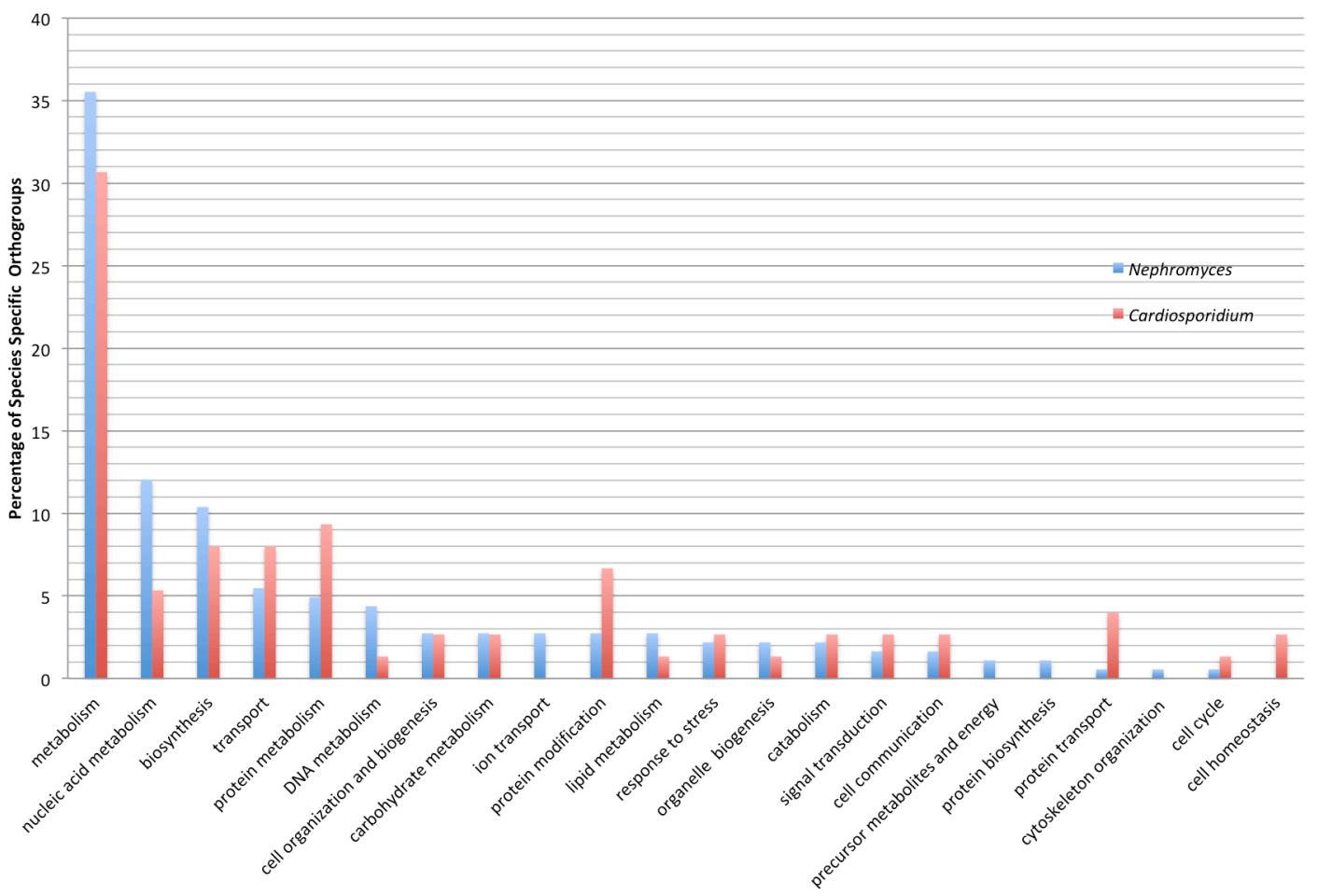

Appendix Figures 1) Comparison of biological process GOSlim terms from lineage specific orthologous genes from both Nephromyces (blue) and Cardiosporidium (red), respectively. GOSlim terms are grouped by their major function, showing no clear pattern of gene losses by functional category between Cardiosporidium and Nephromyces. 


\section{Molecular function GOSlim}

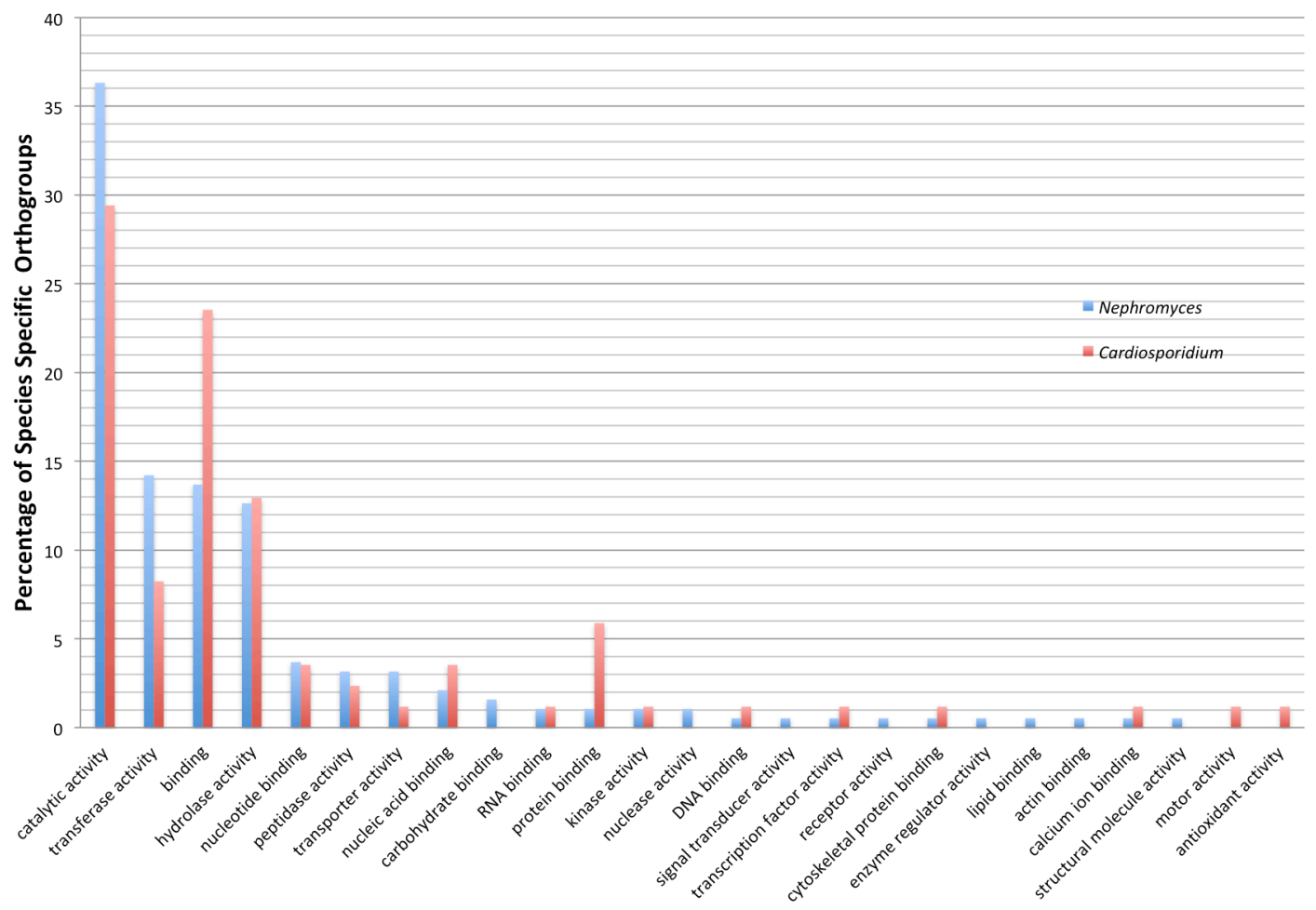

Appendix Figure 2) Comparison of molecular function GOSlim terms from lineage specific orthologous genes from both Nephromyces (blue) and Cardiosporidium (red), respectively. GOSlim terms are grouped by their major molecular function, showing no clear pattern of gene losses by functional category between Cardiosporidium and Nephromyces. 